\title{
STRATEGIES TO IMPROVE THE DIAGNOSIS AND TREATMENT OF ISCHEMIA-REPERFUSION INJURY AFTER LUNG TRANSPLANTATION
}

Eric Joseph Charles

Charlottesville, Virginia

Bachelor of Science, Pennsylvania State University, 2007

Doctor of Medicine, Drexel University College of Medicine, 2012

\begin{abstract}
A Dissertation presented to the Graduate Faculty of the University of Virginia in Candidacy for the Degree of Doctor of Philosophy
\end{abstract}

Department of Molecular Physiology and Biological Physics

\section{University of Virginia}

December 2017 


\section{TABLE OF CONTENTS}

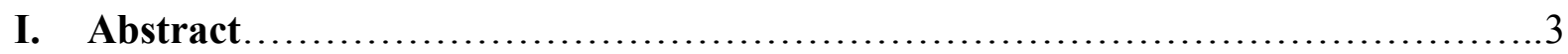

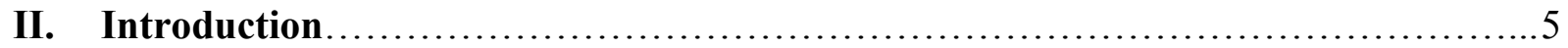

a. Lung Transplantation and Current Limitations.................................

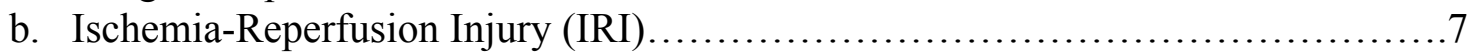

c. Ex vivo Lung Perfusion (EVLP)......................................... 9

d. Imaging Methods to Diagnose Lung Injury................................. 10

e. Research Objectives................................................... 11

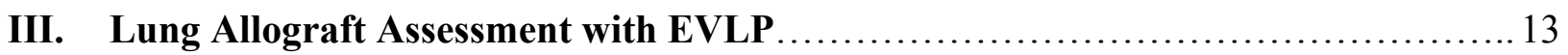

a. PROJECT \#1. Donation After Circulatory Death Lungs Transplantable Up to Six Hours After Ex Vivo Lung Perfusion................................ 14

b. PROJECT \#2. Ex Vivo Assessment of Donation after Circulatory Death Lungs Exposed to Increasing Warm Ischemia Time.......................... 33

IV. Noninvasive Diagnosis of Lung IR Injury by SPECT Imaging ...................55

a. PROJECT \#3. Use of cFLFLF, a Formyl Peptide Receptor Ligand, and SPECT Imaging to Noninvasively Diagnose and Monitor Lung IschemiaReperfusion Injury................................................. 56

V. Molecular Targets to Attenuate Lung IR Injury............................ 78

a. PROJECT \#4. Lungs Donated After Circulatory Death and Prolonged Warm Ischemia are Successfully Transplanted after Enhanced Ex Vivo Lung Perfusion Using Adenosine A2B Receptor Antagonism. .79

b. PROJECT \#5. TRPV4 Channels Mediate Pulmonary Inflammation During IRI.

c. PROJECT \#6. Pannexin-1 Channels on Endothelial Cells Mediate Vascular Inflammation During Lung IRI 104

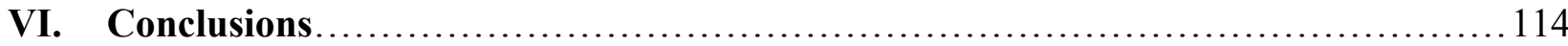

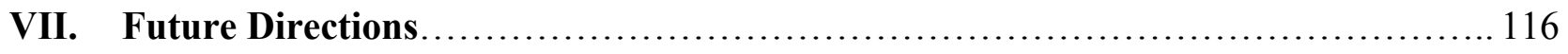

VIII. Acknowledgements.................................................. 120

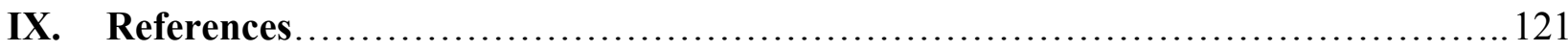




\section{Abstract}

Lung transplantation has advanced dramatically since its inception in 1963, but still lags behind other solid organs in terms of donor organ utilization and transplant success rates. Increased use of available donor lungs, development of noninvasive techniques for early diagnosis of primary graft dysfunction (PGD), and the design of therapies to attenuate ischemiareperfusion injury (IRI) are needed to improve the rates and outcomes of lung transplantation.

My graduate research entailed various projects that addressed three critical areas of lung transplantation. I first focused on the role of ex vivo lung perfusion (EVLP) as a platform to assess the effects of cold and warm ischemia on allograft function. High rates of lung IRI and PGD after transplantation of marginal donor lungs leads to poor outcomes and thus a reluctance among transplant centers to utilize these available organs. Using a preclinical porcine model of lung transplantation, we demonstrated that marginal donor lungs (procured after circulatory death) exposed to 6 hours of cold ischemia after EVLP can still be successfully transplanted. These findings suggest that organ allocation can be improved without compromising allograft function using a strategy that combines ex vivo assessment and rehabilitation with cold preservation. Another project that I completed explored the effect of increasing warm ischemia time (WIT) on allograft function and demonstrated that longer WIT does not predict worse lung function when lungs are assessed on EVLP. Expanding acceptable WIT after circulatory death may eventually allow for inclusion of uncontrolled donation after circulatory death (DCD) lungs (lungs procured from unplanned donors) in procurement protocols.

Considering that IRI leads to PGD and early morbidity and mortality after lung transplantation, I next explored mechanisms by which in vivo leukocyte labeling could be used to noninvasively image cell-type specific lung inflammation, thus improving and expediting 
diagnosis. Polymorphonuclear leukocytes (PMNs) traffic to the lungs early during acute lung injury, infiltrate in abundance, and are known to express high levels of formyl peptide receptor 1 (FPR1), which aids in chemotaxis. Thus, we established a diagnosis technique utilizing a FPR1 peptide ligand conjugated with technetium-99m $\left({ }^{99 \mathrm{~m}}\right.$ Tc-cFLFLF) and single-photon emission computed tomography (SPECT) that enabled quantifiable, noninvasive imaging diagnosis of lung IRI and allowed for monitoring of injury resolution over time.

Finally, I turned my attention to molecular targets to attenuate lung IRI. We first demonstrated that enhanced EVLP supplemented with an adenosine A2B receptor antagonist could rehabilitate injured DCD lungs and attenuate post-transplant IRI, thus allowing for successful transplantation. Second, we identified transient receptor potential vanilloid 4 (TRPV4) cation channels as important regulators of endothelial cell permeability and epithelial cell activation during lung IRI and demonstrated that antagonism of TRPV4 may be a promising therapy to attenuate lung IRI. Finally, I focused on the role of pannexin 1 (Panx1) channels in lung IRI. We established endothelial cell Panx1 as an important mediator of vascular permeability, pulmonary edema accumulation, and leukocyte infiltration, and we demonstrated that Panx1 inhibitors attenuate IRI and edema and may be another novel therapeutic strategy to improve outcomes after lung transplantation.

Collectively, the work presented here challenges the status quo regarding lung transplantation. Utilization of EVLP to assess and rehabilitate marginal donor lungs, earlier diagnosis of lung IRI via noninvasive ${ }^{99 \mathrm{~m}}$ Tc-cFLFLF SPECT imaging, and the use of novel pharmacologic therapies to attenuate lung IRI may improve outcomes for lung transplant patients. 


\section{Introduction}

\section{Lung Transplant Overview and Current Limitations}

Lung transplantation is a life-saving operation for patients with end-stage pulmonary disease who have failed conservative medical management. [1] Over the past 10 years, lung transplant volume in the United States increased by $66 \%$, from 1,405 transplants in 2006 , to 2,327 transplants in 2016. Unfortunately, wait list additions have increased at a similar rate, with 2,789 new registrations in 2016. [2] As a result of the supply-demand mismatch, wait list mortality remains high, ranging from 5-25 deaths per 100 waitlist years depending on lung disease diagnosis. [3] For patients who are fortunate enough to receive a transplant, 5-year unadjusted overall survival is only 55\%. [3, 4] Compared with other types of solid organ transplantation, lung transplantation has the lowest median survival (Figure 1). [3-5] Despite these shortcomings, more lung transplant recipients are alive in the US (11,486 as of June 2014) than at any other time since the inception of lung transplantation in 1963. [4]

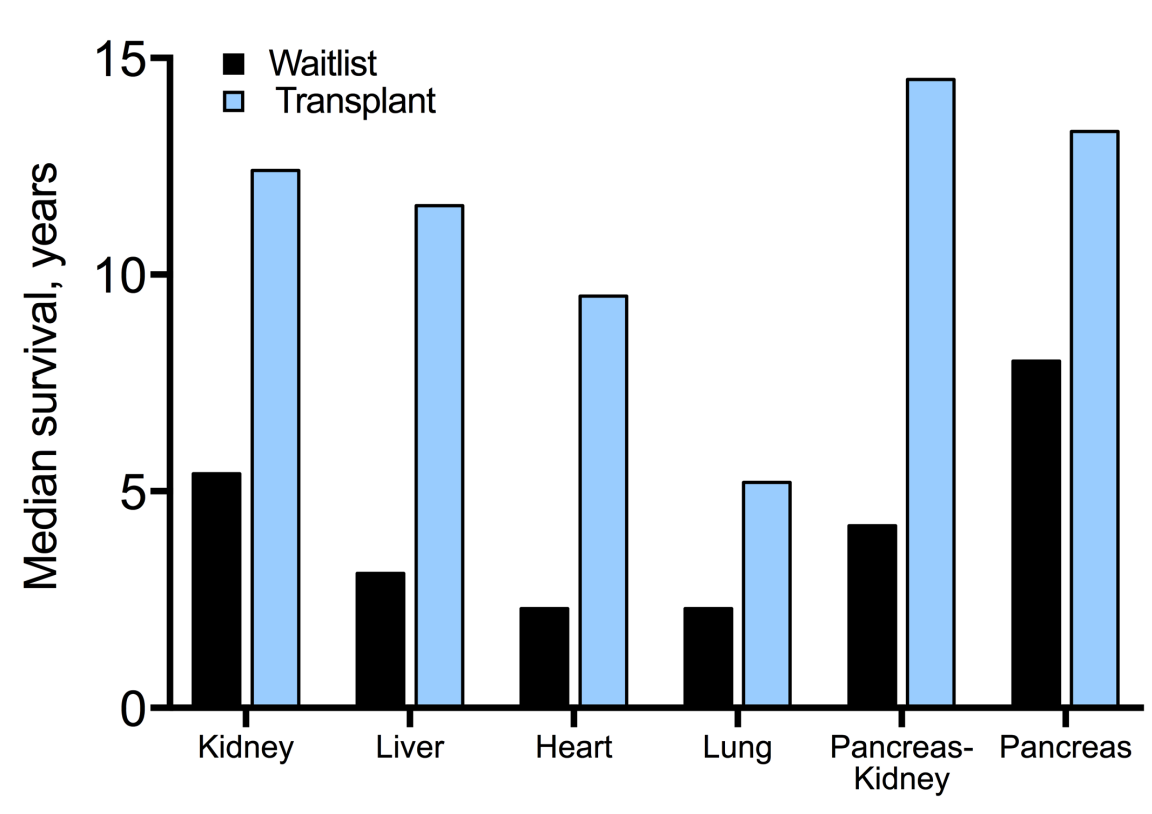

Figure 1: Waitlist and transplant median survival for solid organ transplants in the United States. Data adapted from Rana A et al. [5] 
Many efforts have been made to increase the number of successful lung transplants performed, including the use of marginal donor lungs (such as donation after circulatory death [DCD] lungs), the adoption of ex vivo lung perfusion (EVLP) as a platform to assess donor lung quality and potentially rehabilitate injured lungs, and the identification of pharmacologic targets to attenuate lung IRI and block rejection. Additionally, efforts have been made to incorporate the use of veno-venous extracorporeal membrane oxygenation (ECMO) and lung protective ventilation strategies to support patients through the immediate postoperative period after lung transplantation.

EVLP allows for the assessment and possible rehabilitation of donor lungs by providing physiologic preservation outside of the body prior to transplant. [6] Most lung transplants are performed with organs from heart-beating, brain-dead donors (donation after brain death [DBD]), which allows for minimization of both cold and warm ischemic times. [7] Use of nonheart-beating, DCD lungs is much less common due to higher rates of ischemia-reperfusion injury (IRI) and primary graft dysfunction (PGD). As the supply of traditional DBD lungs remains stagnant and wait list mortality continues to rise, DCD lungs have become an important source of additional organs. [3] Studies to date suggest that outcomes with Maastricht category III DCD lungs (i.e. in-hospital patients awaiting cardiac arrest) are similar to outcomes with DBD lungs. [8-10] Even with inclusion of DCD lungs in transplant protocols, overall utilization remains low, and the supply of acceptable organs has not fulfilled the ever-increasing demand. $[11,12]$

Currently, DCD lungs are used in less than $2 \%$ of lung transplants per year due to the increased risk of PGD. [3] PGD, which typically results from severe IRI, is a clinical diagnosis that describes acute lung injury that occurs within the early postoperative period (72 hours) after 
lung transplantation and is characterized by reduced oxygenation capacity and progressive lung failure.[13] PGD occurs in up to $30 \%$ of patients and is the leading cause of early morbidity and mortality.[13-15] Rates of late organ failure (bronchiolitis obliterans and chronic rejection) are also significantly higher in patients diagnosed with PGD.[16] The use of EVLP prior to transplantation may help alleviate the resultant IRI and lower the incidence of PGD. [17] Thus, one focus of my graduate research was to determine if EVLP could be used to perform marginal donor lung assessment followed by targeted therapeutic rehabilitation, allowing for successful transplantation of otherwise unacceptable lungs.

The International Society for Heart and Lung Transplantation grading system for severity of PGD uses the allograft's oxygenation capacity (ratio of partial pressure of oxygen in arterial blood to the fraction of inspired oxygen $\left[\mathrm{PaO}_{2} / \mathrm{FiO}_{2}\right]$ ) and the presence of infiltrates on chest radiograph, both of which are general markers of lung dysfunction. [14] There are no clinically available methods for in vivo leukocyte labeling that can identify and noninvasively image celltype specific inflammation after lung transplantation.[18] This inability to diagnose IRI early may contribute to high rates of morbidity and mortality. Strategies are needed that allow for more accurate diagnosis, earlier initiation of targeted treatment strategies, and an ability to monitor resolution of injury over time. Thus, a second focus of my graduate research was the use of an in vivo leukocyte labeling method to develop a noninvasive diagnostic technique utilizing SPECT imaging.

\section{Ischemia-Reperfusion Injury}

The underlying pathophysiology responsible for PGD is post-transplant IRI. [19] Lung IRI involves resident alveolar macrophage activation, neutrophil infiltration, endothelial cell 
disruption, alveolar epithelial cell apoptosis, and activation of invariant natural killer T-cells

(Figure 2). [20-25] The initial ischemic insult results in hypoxia and cessation of cyclic mechanotransduction, causing vascular endothelial cells and resident alveolar macrophages to generate reactive oxygen species (ROS) and proinflammatory cytokines. [26] IL-8, IL-12, IL-17, IL-18, and TNF- $\alpha$, as well as damage associated molecular pattern (DAMP) molecules (e.g. HMGB1 and extracellular ATP) are generated, along with activation of multiple inflammatory signaling pathways involving calcium/calmodulin-dependent nitric oxide synthase (NOS), NADPH oxidase, and nuclear factor-kappa B (NF-kB). [22, 26-32] Expression of endothelial cell adhesion molecules is up-regulated, leading to leukocyte attraction and migration into the interstitium upon reperfusion and perpetuation of further tissue damage. [33]

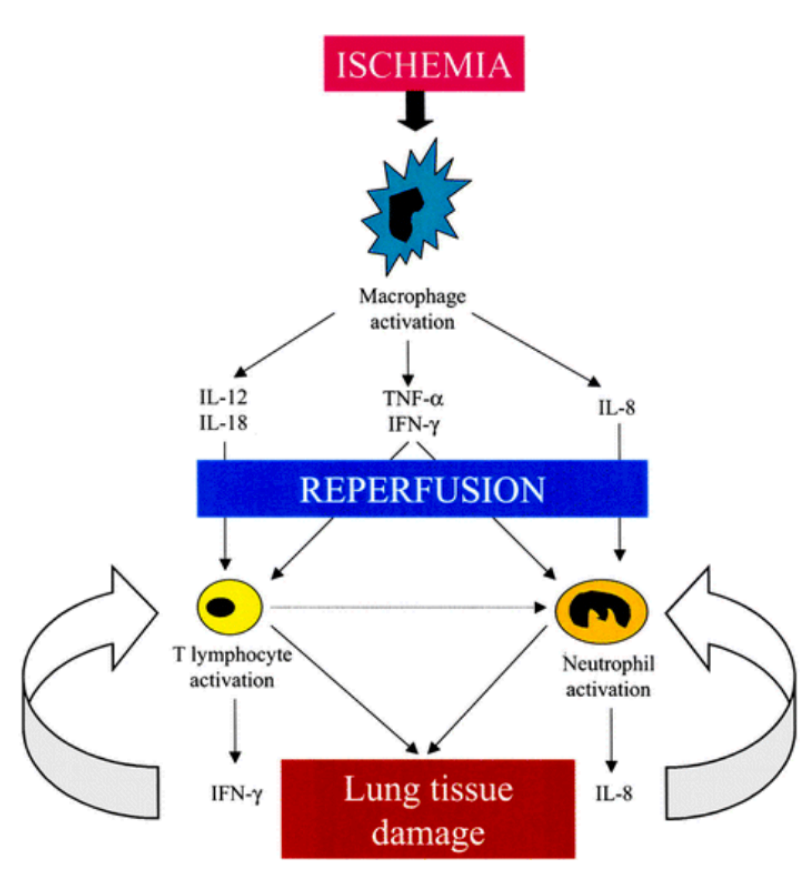

Figure 2: Mechanisms of lung ischemia-reperfusion injury as illustrated by de Perrot $\mathrm{M}$ et al. [28] Ischemia results in macrophage activation, followed by reperfusion which activates T-cells and neutrophils, with all involved cell types contributing to lung tissue damage. 
When reperfusion occurs, the initial injury caused by ischemia is amplified as a result of significant ROS production. The oxidative stress caused by reperfusion leads to gene transcription and increased translation of cell-surface adhesion proteins. Neutrophil activation and sequestration in the lung leads to additional ROS production and positive feedback on the entire lung IRI mechanism. Neutrophils are a key component of the lung tissue damage that occurs. When activated, neutrophils release proinflammatory cytokines, ROS, chemoattractants for other inflammatory cells, proteases, and elastase.

Clinically, lung IRI is characterized by increased vascular permeability and resistance, accumulation of pulmonary edema, poor gas exchange, reduced oxygenation capacity, and increased pulmonary artery (PA) pressure. [26] Impaired oxygenation and radiographic evidence of pulmonary edema occurs in up to $50 \%$ of lung transplant patients, illustrating the frequency with which lung IRI occurs. [34] When these physiologic changes occur within 72 hours posttransplant (definition of PGD), the 30-day rate of all-cause mortality is $63 \%$, compared with $8 \%$ for patients without PGD (relative risk 7.15). [34] Lung IRI has a significant impact on lung transplant outcomes thus highlighting the need for improved diagnosis techniques and treatment strategies. Therefore, another focus of my research was to identify novel molecular targets to attenuate lung IRI, reduce the incidence of PGD, and improve allograft function.

\section{Ex vivo lung perfusion (EVLP)}

In contrast to standard cold preservation of lungs after procurement, EVLP is a technique used to maintain lungs in a normothermic, physiologic state prior to transplantation. [35] Lung tissue remains metabolically active and viable while on EVLP, allowing for functional assessments and reconditioning. The main objectives of EVLP are to reduce pulmonary edema 
by perfusing the lungs with high oncotic solutions, remove donor leukocytes that contribute to post-transplant inflammation, and improve ventilation/perfusion matching with lung recruitment ventilation strategies. [35] Additional treatments that can be delivered during EVLP are being investigated, such as IL-10 gene therapy, beta-adrenoreceptor agonist inhalation, and the delivery of high-dose anti-infective agents. $[17,36,37]$ The true promise of EVLP is in developing methods to rehabilitate damaged organs back to a physiologic state where they can be successfully transplanted.

\section{Imaging Methods to Diagnose Lung Injury}

While the goal of EVLP is to recondition damaged organs and allow for successful transplantation, the occurrence of some degree of IRI is inevitable with the use of marginal organs. Methods to accurately diagnose IRI early after transplantation are needed so that treatments can be initiated, thus preventing the progression to PGD. Current imaging modalities (plan radiographs, computed tomography [CT] scans, and magnetic resonance imaging [MRI]) are important for diagnosing lung diseases by providing complementary anatomic and structural data to physical exam findings and laboratory blood results, however they are non-specific and often do not allow for the discrimination between various diseases. Novel molecular imaging methods are needed that would permit early, cell-type specific diagnoses. When paired with in vivo leukocyte labeling, nuclear imaging modalities such as single-photon computed tomography (SPECT) and positron emission tomography (PET) have high sensitivity and resolution and may allow for more accurate diagnosis of lung diseases, such as IRI after lung transplantation. 


\section{Research Objectives}

The research presented here aims to address three key areas of lung transplantation: marginal donor lung utilization, early and accurate diagnosis techniques for lung IRI, and pharmacologic therapies for attenuation of lung IRI. The first focus of study was to evaluate the role of EVLP as a platform to assess the effects of cold and warm ischemia on allograft function. Specifically, we tested the hypothesis that marginal donor lungs exposed to 6 hours of cold ischemia after EVLP can still be successfully transplanted. This would open the door for expanding the concept of EVLP centers, where marginal donor organs would arrive to be reconditioned with EVLP, preserved cold, and then distributed regionally or even nationwide to the most appropriate recipient. Additionally, we sought to evaluate the impact of increasing warm ischemia time after circulatory death on allograft function to determine if current guidelines for transplant acceptability after EVLP are appropriate. Acceptable warm ischemia time criteria may be too stringent thus limiting the utilization of marginal donor lungs.

The second focus of study was to develop a noninvasive method to diagnosis lung IRI early with cell-type specific molecular imaging. Patients with lung IRI that classifies as PGD have significantly higher mortality rates compared with patients without lung IRI. Considering that PMNs are an important contributor to acute lung injury and are responsible for lung tissue damage, we focused on FPR1, a chemotactic cell-surface receptor upregulated on activated

neutrophils. Thus we tested the hypothesis that ${ }^{99 \mathrm{~m}} \mathrm{Tc}-\mathrm{cFLFLF}$, which binds to FPR1 on activated neutrophils, could serve as a cell-specific molecular probe to noninvasively diagnose and monitor lung IRI via SPECT imaging.

The third focus of study sought to identify novel mechanisms of lung IRI that would lead to novel pharmacologic targets that attenuate lung IRI. To this end we hypothesized that 
adenosine A2B receptor (A2BR), pannexin 1 (Panx1) channels, and transient receptor potential vanilloid 4 (TRPV4) channels all significantly contribute to lung IRI and that antagonism of these membrane molecules would attenuate lung IRI. We first explored a strategy of ex vivo treatment of marginal donor lungs utilizing ATL802, an A2BR antagonist, to enhance the rehabilitative capacity of EVLP. Next, we identified TRPV4 cation channels as important regulators of endothelial cell permeability and epithelial cell activation and demonstrated that administration of GSK2193874, a TRPV4 specific antagonist, mitigates lung dysfunction and edema accumulation in a murine model of lung IRI. Lastly, we evaluated the contribution of endothelial cell Panx1 channels in the development of lung IRI and demonstrated that pharmacologic inhibition of Panx1 with probenecid or carbenoxolone is protective to lungs after IR.

Collectively, the presented research provides a comprehensive approach to improve the understanding, diagnosis, and treatment of lung IRI. Incorporation of EVLP, cell-specific molecular imaging modalities, and novel pharmacologic therapies may lead to a reduction in the incidence and severity of lung IRI and an improvement in lung transplant outcomes. 


\section{Lung Allograft Assessment with Ex Vivo Lung Perfusion (EVLP)}

EVLP as a platform to assess and recondition donation after circulatory death (DCD) lungs is investigated, with specific attention to the effects of cold ischemia after EVLP and increasing warm ischemia time prior to EVLP on allograft function. 


\title{
PROJECT \#1
}

\section{Donation After Circulatory Death Lungs Transplantable Up to Six Hours After Ex Vivo Lung Perfusion}

\author{
Eric J Charles $\mathrm{MD}^{1}$, Mary E Huerter MD MA ${ }^{1}$, Cynthia E Wagner MD ${ }^{1}$, \\ Ashish K Sharma MBBS $\mathrm{PhD}^{1}$, Yunge Zhao MD $\mathrm{PhD}^{1}$, Mark H Stoler MD², \\ J Hunter Mehaffey MD ${ }^{1}$, James M Isbell MD MSCI ${ }^{1}$, Christine L Lau MD MBA ${ }^{1}$, \\ Curtis G Tribble $\mathrm{MD}^{1}$, Victor E Laubach $\mathrm{PhD}^{1}$, Irving L Kron $\mathrm{MD}^{1}$ \\ Departments of ${ }^{1}$ Surgery and ${ }^{2}$ Pathology, \\ University of Virginia, Charlottesville, Virginia
}

\section{Status of Research: PUBLISHED}

Charles EJ, et al. Annals of Thoracic Surgery. 2016; 102(6):1845-53.

PMID: 27614736 


\section{Abstract}

Background: Despite the critical need for donor lungs, logistical and geographical barriers hinder lung utilization. We hypothesized that donation after circulatory death (DCD) lungs subjected to 6-hours of cold preservation after ex vivo lung perfusion (EVLP) would have similar outcomes after transplantation compared to lungs transplanted immediately after EVLP, and both would perform superiorly compared with lungs transplanted immediately after procurement. Methods: Donor porcine lungs were procured after circulatory death and 15-minutes of warm ischemia. Three groups ( $\mathrm{n}=5$ /group) were randomized: immediate left lung transplantation (Immediate), EVLP for 4-hours followed by transplantation (EVLP), or EVLP for 4-hours followed by 6-hours of cold preservation followed by transplantation (EVLP+Cold). Lungs were reperfused for 2-hours prior to obtaining pulmonary vein samples for $\mathrm{PaO}_{2} / \mathrm{FiO}_{2}$ calculations, airway pressures for compliance measurements, and wet/dry weight ratios.

Results: $\mathrm{PaO}_{2} / \mathrm{FiO}_{2}$ ratios in EVLP and EVLP+Cold groups were significantly improved compared with the Immediate group (429.7 \pm 51.8 and $436.7 \pm 48.2$ versus $117.4 \pm 22.9 \mathrm{mmHg}$, respectively). Additionally, dynamic compliance was significantly improved in the EVLP and EVLP+Cold groups compared to Immediate group $(26.2 \pm 4.2$ and $27.9 \pm 3.5$ versus $11.1 \pm 2.4$ $\mathrm{mL} / \mathrm{cmH}_{2} \mathrm{O}$, respectively). There were no differences in oxygenation capacity or dynamic compliance between EVLP and EVLP+Cold groups. Inflammatory cytokine levels were significantly lower in EVLP and EVLP+Cold groups.

Conclusions: DCD lungs can be successfully transplanted up to 6-hours after EVLP. Cold preservation of lungs following ex vivo assessment and rehabilitation may improve organ allocation, even to distant recipients, without compromising allograft function. 


\section{Introduction}

Lung transplantation remains the standard of treatment for end-stage pulmonary disease.

[1] The number of transplants performed per year has increased over the past 10-years;

unfortunately the waiting list has increased more rapidly. [3] Recent modifications have been made in the allocation scoring system [3], as well as improvements in the use of donation after circulatory death (DCD) organs [10], ex vivo lung perfusion (EVLP) [38, 39], and drug-directed lung rehabilitation. $[40,41]$ Due to the risk of primary graft dysfunction, criteria for procurement are conservative and surgeons are reluctant to transplant marginal lungs. [19] Increasing the availability of donor lungs and improving organ assessment may allow more lung transplants to occur. [17]

The use of DCD organs has become more common, but constitutes only a fraction of all transplants. [42] Lungs are utilized from eligible multi-organ donors only $15-20 \%$ of the time, and few DCD lungs are procured and transplanted ( $<2 \%$ of all lung transplants). [3] According to the United Network of Organ Sharing, organs were procured and transplanted from 1205 DCD donors in 2013, mostly organs other than lungs. [43] If lungs were utilized from the same number of DCD donors, the number of lung transplants per year could increase by $50 \%$ (based on data from the U.S. Department of Health and Human Services Organ Procurement and Transplantation Network which lists 2,057 as the number of lung transplants performed in the U.S. in 2015). [44] Using EVLP as a platform for assessment and rehabilitation allows for procurement of lungs that would otherwise be rejected. Allocation to the most suitable recipient, either local or distant, can occur after a determination of quality has occurred. Increasing the number of donor lungs that are procured and assessed with EVLP may increase the number of lung transplants performed each year, leading to a decrease in wait list times and mortality. 
The purpose of the current study was to determine the effect of prolonged cold preservation after EVLP rehabilitation of DCD lungs on outcomes after transplantation, using a porcine model. We hypothesized that lungs undergoing 6-hours of cold preservation after EVLP would have similar outcomes after transplantation compared to lungs transplanted immediately after EVLP, and both would perform superiorly compared with DCD lungs transplanted immediately after procurement. An additional period of 6-hours of cold preservation would allow for lung allocation to a recipient anywhere in the U.S. regardless of donor location. At the completion of EVLP, the most suitable recipient would be identified and called in to their local hospital for transplant preparation, while the organ is packaged cold and transported from the EVLP center to the recipient hospital.

\section{Materials and Methods}

\section{Animals and Study Groups}

This study complied with the 1996 Guide for the Care and Use of Laboratory Animals as recommended by the U.S. National Institutes of Health. The University of Virginia Animal Care and Use Committee approved the study protocol and all animals received humane care.

Mature domestic swine of both sexes $(25-41 \mathrm{~kg})$ were randomized to three groups ( $\mathrm{n}=5$ /group) to reflect three unique transplant scenarios. All donor swine underwent hypoxic cardiac arrest, followed by 15-minutes of warm ischemia prior to cold preservation flush and procurement. For Immediate group, donor lungs were procured, maintained cold during backtable preparation of the left lung, and transplanted into size-matched recipients. The Immediate group served as the non-EVLP control group. For EVLP group, lungs were procured and underwent 4-hours of EVLP, followed by back-table left-lung preparation and transplantation. 
The third group, EVLP+Cold, underwent procurement and 4-hours of EVLP, followed by 6hours of $4{ }^{\circ} \mathrm{C}$ cold preservation. The left lung was then prepared and transplanted. All recipient animals underwent 2-hours of in vivo reperfusion.

\section{Donor Lung Procurement}

Donor lungs were procured using a standard protocol as previously described. [45] Swine were anesthetized with ketamine $(50 \mathrm{mg} / \mathrm{kg})$ and xylazine $(5 \mathrm{mg} / \mathrm{kg})$, intubated, and ventilated. General anesthesia was maintained with 3\% isoflurane and 100\% fraction of inspired oxygen $\left(\mathrm{FiO}_{2}\right)$. Animals were ventilated with a tidal volume of $8 \mathrm{~mL} / \mathrm{kg}$, respiratory rate $15-20$ breaths/minute, and positive end-expiratory pressure $5.0 \mathrm{cmH}_{2} \mathrm{O}$. After 10-minutes of oxygenation, initial donor arterial blood gas measurement was performed. Systemic heparin was not administered to donor animals. All swine had continuous electrocardiogram monitoring. The endotracheal tube was subsequently clamped and the animal was euthanized by hypoxic cardiac arrest. Prior to initiation of the procurement operation, all animals underwent 15-minutes of warm ischemia from the time asystole was noted on the monitor and confirmed by the absence of auscultatory heart tones. Ventilation with $100 \% \mathrm{FiO}_{2}$ was resumed for the final 5-minutes of the warm ischemia period. A median sternotomy was performed, followed by pericardiotomy. A cardioplegia cannula (Terumo Heart Inc., Ann Arbor, MI) was placed into the main pulmonary artery (PA) and $500 \mu \mathrm{g}$ of Prostaglandin-E1 (Pfizer Inc., New York, NY) was injected. The left atrial (LA) appendage was incised and both vena cava were ligated. Flush with 1.5 liters cold Perfadex ${ }^{\circledR}$ (XVIVO Perfusion Inc., Englewood, CO) supplemented with 15,000 IU of heparin (Hospira Inc., Lake Forest, IL) was performed. The thoracic cavity was filled with ice slush. At the completion of the flush, the trachea was clamped and the heart and lungs explanted. 
The heart was removed, leaving a generous LA cuff. For Immediate group, lungs were separated and back-table preparation of the left bronchus, PA, and LA cuff was completed. Flush was performed retrograde with cold Perfadex $(500 \mathrm{~mL})$ supplemented with heparin to remove additional blood clots. For EVLP and EVLP+Cold groups, preparation of the trachea, main PA, and LA cuff was completed to allow for EVLP.

\section{EVLP}

EVLP was performed for groups EVLP and EVLP+Cold. [46, 47] A green cannula (XVIVO Perfusion Inc., Englewood, CO) was sewn to the LA cuff, a yellow cannula (XVIVO Perfusion Inc., Englewood, CO) was secured within the main PA, and 7-0 endotracheal tube was secured within the trachea. Retrograde flush was performed using cold Perfadex $(500 \mathrm{~mL})$.

The EVLP circuit was assembled as previously described [38] with use of a perfusion reservoir, heat exchanger, and pump, and primed with Steen Solution ${ }^{\text {TM }}$ (XVIVO Perfusion Inc, Englewood, CO) supplemented with $500 \mathrm{mg}$ cefazolin (APP Pharmaceuticals, Schaumburg, IL), 500 mg methylprednisolone (Pfizer Inc., New York, NY), and 10,000 IU heparin. Flow was initiated through the lungs at $0.2 \mathrm{~mL} / \mathrm{min}$. LA pressure was maintained between 0 and $5 \mathrm{mmHg}$. The Steen was warmed to $37^{\circ} \mathrm{C}$, flow was slowly titrated up to $40 \%$ of estimated cardiac output (100 mL/kg donor body weight), and ventilation with room air was initiated when the perfusate reached $32^{\circ} \mathrm{C}$ (tidal volume $8 \mathrm{~mL} / \mathrm{kg}$, respiratory rate 8 breaths $/$ minute, positive end-expiratory pressure $5.0 \mathrm{~cm} \mathrm{H}_{2} \mathrm{O}$ ). The perfusate was deoxygenated using a tri-gas mixture ( $86 \%$ nitrogen, $8 \%$ carbon dioxide, $6 \%$ oxygen). Normothermic EVLP was performed continuously for 4-hours. Every hour, following a 15 -minute challenge period with $100 \% \mathrm{FiO}_{2}$, perfusate samples were collected from the PA and LA to measure the partial pressure of oxygen $\left(\mathrm{PaO}_{2}\right)$ and mean, peak, 
and plateau airway pressures were recorded. At completion of EVLP, lungs were flushed anterograde with cold Perfadex $(500 \mathrm{~mL})$ and back-table preparation of the left lung was completed.

\section{Left Lung Transplantation}

Left lung transplantation was performed as previously described. [40] Recipient animals were anesthetized and prepped for left lateral thoracotomy. PA and arterial catheters were placed. Lidocaine $(50 \mathrm{mg}$ ) and heparin (5000 IU) were administered and left pneumonectomy was performed. The donor lung was transplanted into the recipient as follows: end-to-end bronchial anastomosis, end-to-end PA anastomosis, and LA cuff to recipient LA appendage, all with running Prolene sutures (Ethicon Inc., Somerville, NJ). The transplanted lung was reperfused and ventilated.

\section{Reperfusion Period}

Lungs were reperfused in vivo for 2-hours. Systemic arterial blood gas analysis and airway pressure measurements were performed every 30-minutes, preceded by low-pressure lung recruitment. Normal saline, epinephrine, and sodium bicarbonate were administered as necessary. After the 2-hour reperfusion period, samples were obtained directly from the left pulmonary veins allowing for assessment of left lung oxygenation capacity. The donor lung was explanted and the recipient animal euthanized. 


\section{Tissue Cytokines}

Two tissue samples were obtained from the lower lobe after explantation, flash frozen, and stored at $-80^{\circ} \mathrm{C}$. Samples were homogenized with use of FastPrep ${ }^{\circledR}-24$ (MP Biomedicals, Santa Ana, CA). Determination of total protein concentration was performed using a bicinchoninic acid protein assay (Pierce, Rockford, IL). Samples were diluted to equal protein concentrations ( $50 \mu \mathrm{g} / \mathrm{mL})$ and a commercially available porcine multiplex immunoassay kit (EMD Millipore, Billerica, MA) was used to quantify proinflammatory cytokine concentrations.

\section{Pulmonary Edema}

Fresh tissue samples were obtained after explantation (one from the upper lobe, two from the lower lobe) and weighed. Samples were placed in a vacuum oven until a stable weight was obtained. Wet/dry weight ratios were calculated as an indication of pulmonary edema.

\section{Histologic Lung Injury}

After fresh tissue sampling, the airways of the lower lobe were filled with $10 \%$ buffered formalin and the lung was submerged in formalin for 24-hours. Four samples of peripheral lung tissue were collected, paraffin-embedded, sectioned, and hematoxylin-eosin stained. A blinded pathologist reviewed all four slides per animal and scored each slide on a scale from 0-9 to determine a lung injury severity score. The histopathology score was based on the following three components as previously described [38]: polymorphonuclear cells per 40X high-powered field, alveolar edema, and interstitial inflammation (Table 1). 
Table 1: Lung Injury Severity Score

\begin{tabular}{|l|l|l|l|l|}
\cline { 2 - 5 } \multicolumn{1}{c|}{} & \multicolumn{4}{l|}{ Lung Injury Severity Score } \\
\hline & $\mathbf{0}$ & $\mathbf{1}$ & $\mathbf{2}$ & $\mathbf{3}$ \\
\hline PMNs/HPF & $<5$ & $6-10$ & $11-20$ & $>20$ \\
\hline Alveolar Edema & $<5 \%$ & $6-25 \%$ & $26-50 \%$ & $>50 \%$ \\
\hline $\begin{array}{l}\text { Interstitial } \\
\text { Inflammation }\end{array}$ & None & Minimal & Moderate & Severe \\
\hline
\end{tabular}

$P M N s / H P F$, neutrophils per high-powered field

Additionally, two sections of tissue were assessed by immunohistochemistry staining of neutrophils. [48] Mouse monoclonal anti-porcine neutrophil antibody (MBA Biomedicals, Augst, Switzerland) was used as primary antibody and donkey anti-mouse IgG (Jackson ImmunoResearch Laboratories Inc., West Grove, PA) was used as secondary antibody. An avidin-biotin complex was added and incubated at room temperature for 30-minutes. The immunoreactivity was visualized by incubating the sections with 3,3-diaminobenzidine tetrahydrochloride (Dako Inc., Carpinteria, CA) to produce a brown precipitate, and then counterstained with hematoxylin. Five photographs at $40 \mathrm{X}$ magnification were taken from each of two sections per lung and the number of neutrophils counted by a blinded reviewer.

\section{Statistical Analysis}

One-way analysis of variance was used to determine statistical significance between all three groups and adjusted with Tukey's multiple comparisons correction. Student's t-test was used when comparing differences between two groups. All statistical calculations were performed using Prism 6 (GraphPad Software Inc., La Jolla, CA). Data were reported as mean \pm standard error of the mean, with a p-value of 0.05 . 


\section{Results}

\section{Lung Function}

Post-reperfusion lung function was significantly improved in both EVLP and EVLP + Cold groups compared with Immediate group (Figure 3). Oxygenation was significantly higher (EVLP: $429.7 \pm 51.8$, EVLP+Cold: $436.7 \pm 48.2$ versus Immediate: $117.4 \pm 22.9 \mathrm{mmHg}$, $\mathrm{p}<0.001$ ), as was dynamic compliance (EVLP: $26.2 \pm 4.2$, EVLP+Cold: $27.9 \pm 3.5$ versus Immediate: $\left.11.1 \pm 2.4 \mathrm{~mL} / \mathrm{cmH}_{2} \mathrm{O}, \mathrm{p}<0.025\right)$. No differences in either parameter were observed between groups EVLP and EVLP+Cold.
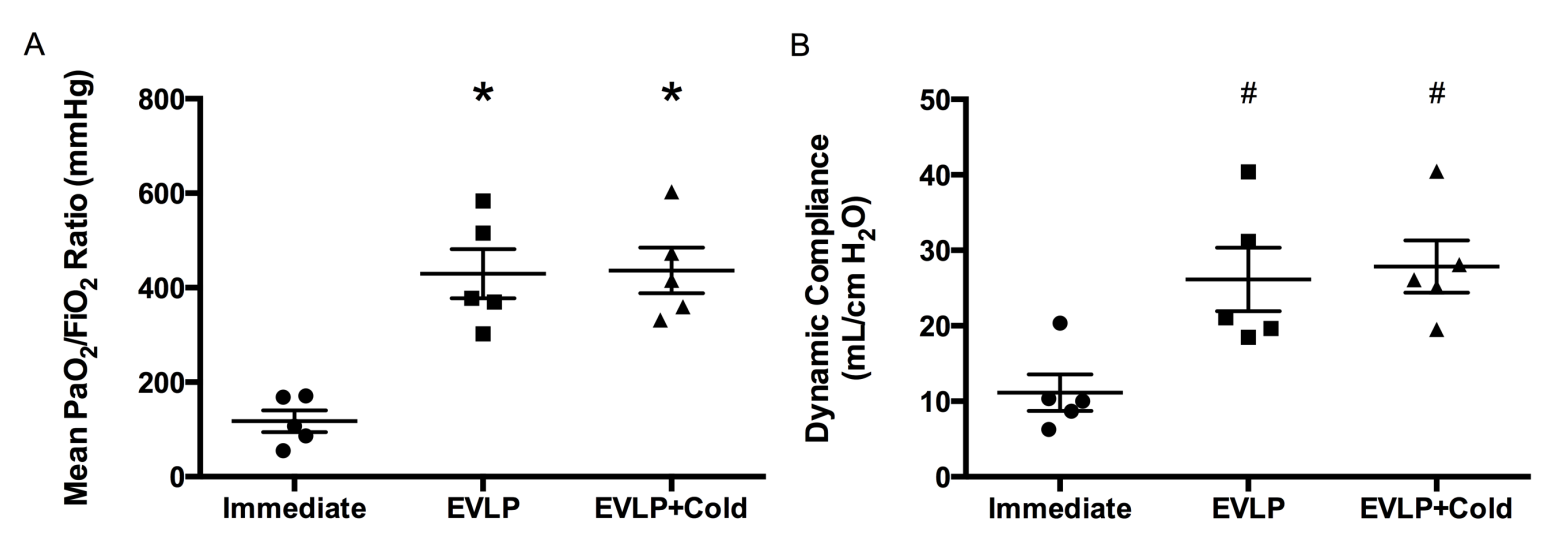

Figure 3: (A) Final oxygenation $\left(\mathrm{PaO}_{2} / \mathrm{FiO}_{2}\right)$ and (B) dynamic compliance at the completion of 2-hours of reperfusion. EVLP, transplantation at the completion of EVLP; EVLP+Cold, 6-hours cold preservation after EVLP and prior to transplantation; Immediate, immediate transplantation without EVLP; $\mathrm{PaO}_{2} / \mathrm{FiO}_{2}$, partial pressure of oxygen/fraction of inspired oxygen. *p $<0.001$ versus Immediate, $\# \mathrm{p}<0.025$ versus Immediate.

At the conclusion of EVLP, there were no differences in $\mathrm{PaO}_{2} / \mathrm{FiO}_{2}$ ratios (EVLP: $529.3 \pm 24.8$ versus $\mathrm{EVLP}+$ Cold: $480.9 \pm 16.0 \mathrm{mmHg}, \mathrm{p}=0.139$ ) or dynamic compliance (EVLP: $22.7 \pm 6.5$ versus EVLP + Cold: $12.7 \pm 0.9 \mathrm{~mL} / \mathrm{cmH}_{2} \mathrm{O}, \mathrm{p}=0.164$ ) (Figure 4). During EVLP,

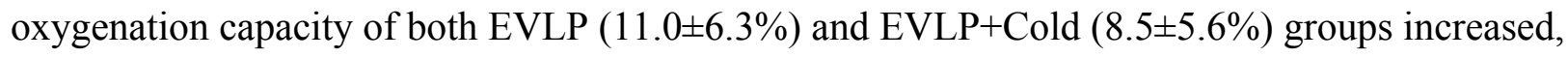
while dynamic compliance increased in the EVLP group (14.8 $\pm 11.0 \%)$, but decreased slightly in EVLP+Cold (-10.4 $\pm 8.0 \%)$ 


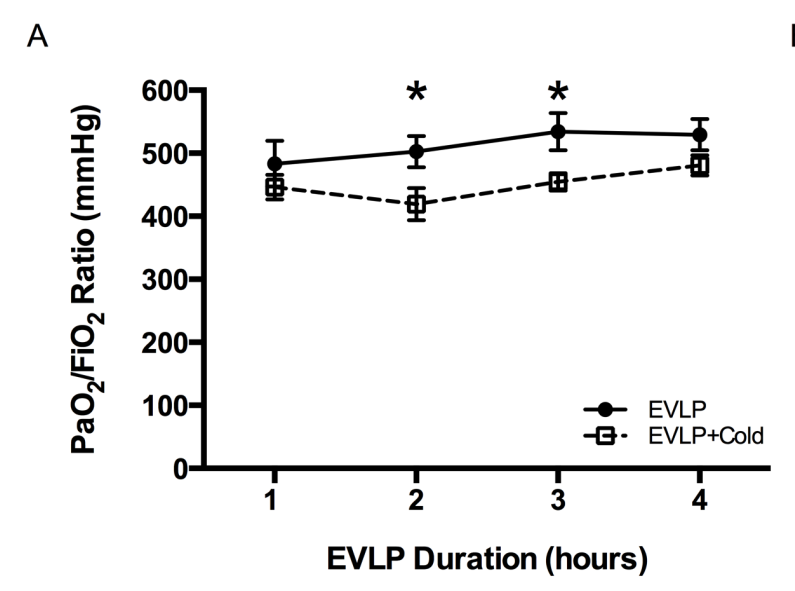

B

C

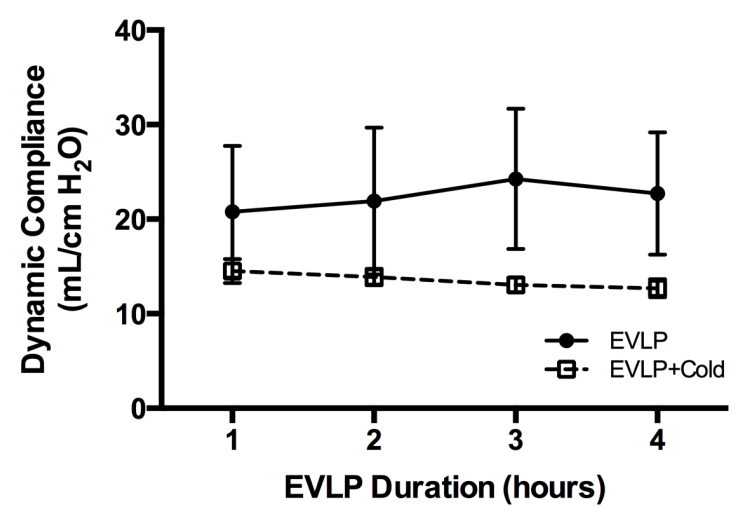

D
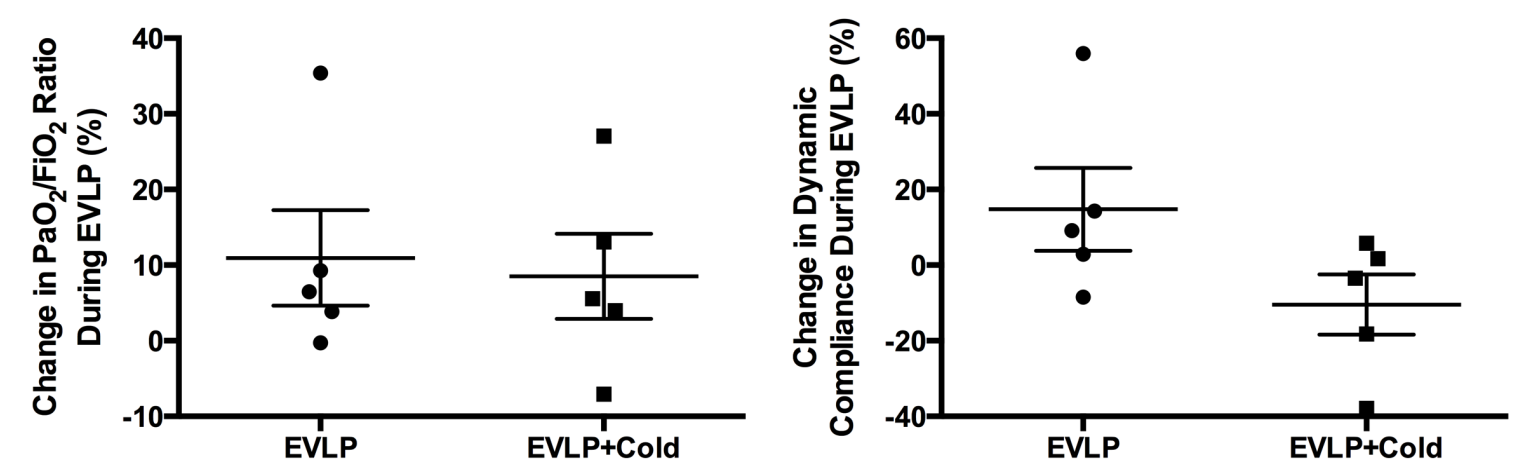

Figure 4: (A) Oxygenation $\left(\mathrm{PaO}_{2} / \mathrm{FiO}_{2}\right)$ and (B) dynamic compliance recorded hourly during EVLP. (C) Change in oxygenation and (D) dynamic compliance from start of EVLP to completion. EVLP, transplantation at the completion of EVLP; EVLP+Cold, 6-hours cold preservation after EVLP and prior to transplantation; $\mathrm{PaO}_{2} \mathrm{FiO}_{2}$, partial pressure of oxygen/fraction of inspired oxygen. ${ }^{*} \mathrm{p}<0.05$

All donor animals had similar pre-hypoxia oxygenation levels (mean $\mathrm{PaO}_{2} / \mathrm{FiO}_{2}$ :

$343.5 \pm 25.3 \mathrm{mmHg}$ ). The mean time-to-death after clamping the endotracheal tube was $24.1 \pm 2.0$ minutes. There were no differences between groups in both starting oxygenation level and timeto-death. Figure 5 shows mean $\mathrm{PaO}_{2} / \mathrm{FiO}_{2}$ ratios from the start of the experiment to conclusion of reperfusion. The Immediate group decreased from a pre-hypoxia value of $315.3 \pm 45.7 \mathrm{mmHg}$ to a post-reperfusion value of $117.4 \pm 22.9 \mathrm{mmHg}$, while the EVLP group increased from 
$292.6 \pm 44.1 \mathrm{mmHg}$ to $429.7 \pm 51.8 \mathrm{mmHg}$ and the EVLP+Cold group increased from $411.3 \pm 22.2$ $\mathrm{mmHg}$ to $436.7 \pm 48.2 \mathrm{mmHg}$.

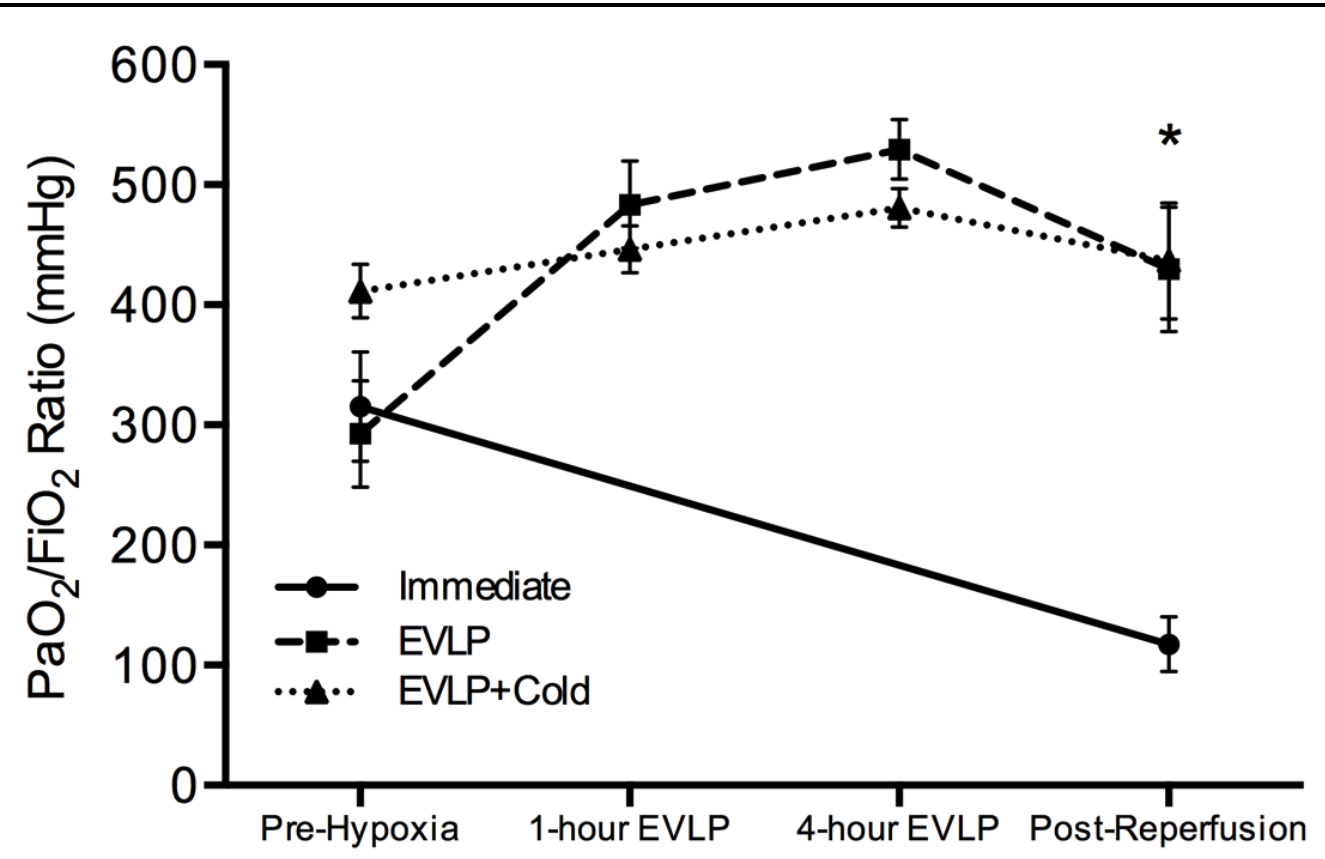

Figure 5: Oxygenation recorded at four time points: donor animal prior to hypoxia (Prehypoxia), 1 hour into EVLP (1-hour EVLP), at the completion of EVLP (4-hour EVLP), and recipient after 2-hours of reperfusion (Post-Reperfusion). EVLP, transplantation at the completion of EVLP; EVLP+Cold, 6-hours cold preservation after EVLP and prior to transplantation; Immediate, immediate transplantation without EVLP; $\mathrm{PaO}_{2} / \mathrm{FiO}_{2}$, partial pressure of oxygen/fraction of inspired oxygen. ${ }^{*} \mathrm{p}<0.001$ for groups EVLP and EVLP+Cold versus Immediate.

\section{Post-Reperfusion Cytokines}

Groups EVLP and EVLP+Cold had consistently and significantly lower tissue levels of cytokines compared with Immediate group (Figure 6). Significant differences were observed for interferon (IFN)- $\gamma$ (EVLP: $322.0 \pm 109.5$, EVLP+Cold: 94.0 \pm 20.3 versus Immediate: $646.0 \pm 61.1$ $\mathrm{pg} / \mathrm{mL}, \mathrm{p}<0.001$ ), interleukin (IL)-1 $\beta$ (EVLP: $412.0 \pm 132.5$, EVLP+Cold: $171.0 \pm 42.2$ versus Immediate: $1193.0 \pm 127.5 \mathrm{pg} / \mathrm{mL}, \mathrm{p}<0.001$ ), and IL-18 (EVLP: 562.0 \pm 290.4, EVLP+Cold: $43.0 \pm 13.0$ versus Immediate: $2142 \pm 354.5 \mathrm{pg} / \mathrm{mL}, \mathrm{p}<0.001)$. The level of IL-6 in EVLP+Cold 
group was significantly lower than Immediate group (EVLP+Cold: 212.0 099.7 , Immediate: 836.0 $\pm 204.4 \mathrm{pg} / \mathrm{mL}, \mathrm{p}=0.03$ ) but the difference between EVLP and Immediate groups was not significant. There were no significant differences in expression of IFN- $\gamma$, IL-1 $\beta$, IL-6, or IL-18 between groups EVLP and EVLP+Cold.

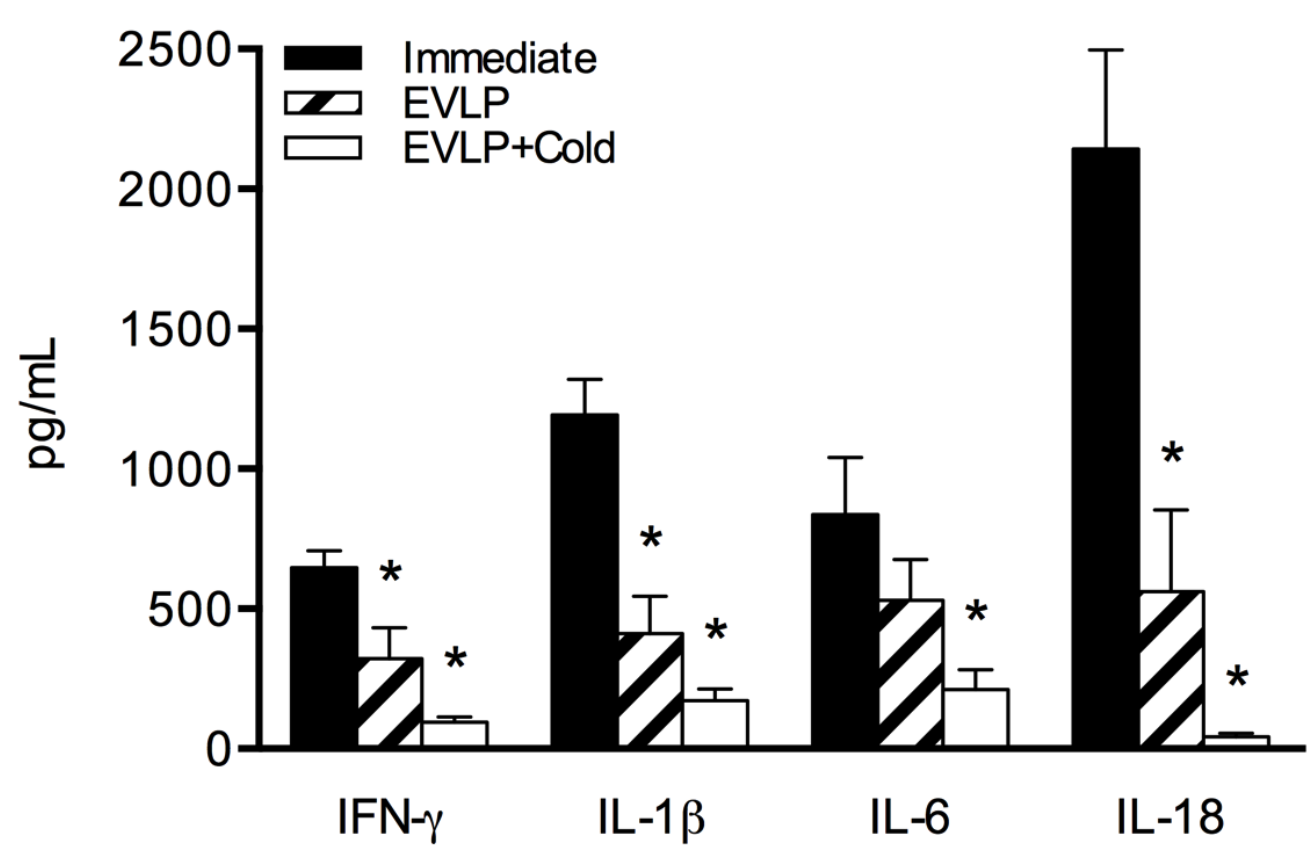

Figure 6: Tissue cytokine levels after reperfusion. EVLP, transplantation at the completion of EVLP; EVLP+Cold, 6-hours cold preservation after EVLP and prior to transplantation; Immediate, immediate transplantation without EVLP. ${ }^{*} \mathrm{p}<0.05$ versus Immediate.

\section{Pulmonary Edema}

Wet/dry weight ratios were calculated from fresh lung tissue. Samples were taken from five recipient pneumonectomy $(\mathrm{PN})$ lungs to determine a baseline wet/dry weight ratio. A significant difference was observed between the wet/dry weight ratio from Immediate group compared to PN group (7.68 \pm 0.39 versus $5.61 \pm 0.30$, respectively, $\mathrm{p}=0.01)$ (Figure 7). A trend towards less pulmonary edema was identified when comparing EVLP $(7.03 \pm 0.57, \mathrm{p}=0.67)$ and EVLP + Cold $(6.76 \pm 0.27, \mathrm{p}=0.39)$ groups to Immediate group (Figure 5). 


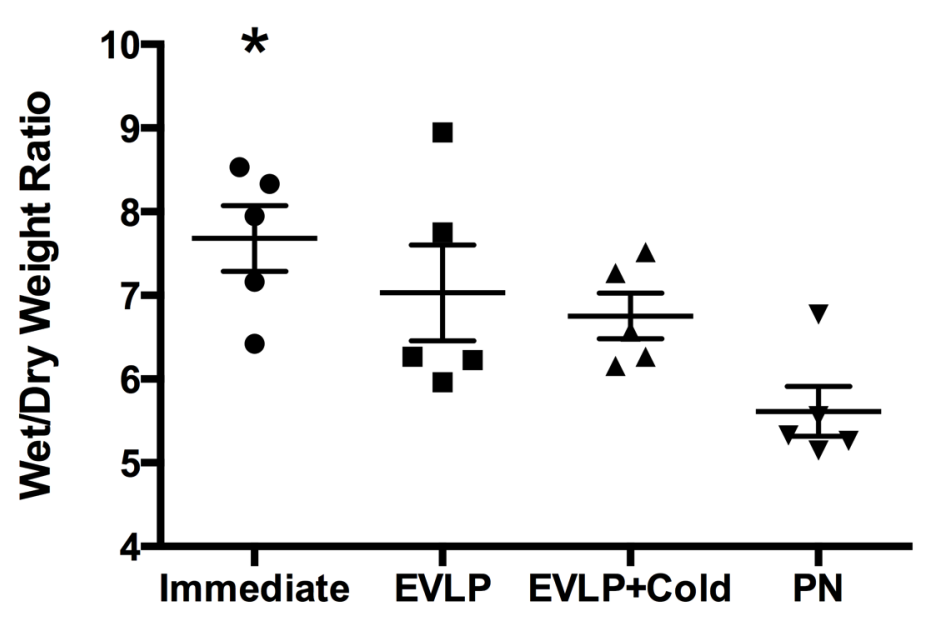

Figure 7: Pulmonary edema after reperfusion determined by tissue wet/dry weight ratios. EVLP, transplantation at the completion of EVLP; EVLP+Cold, 6-hours cold preservation after EVLP and prior to transplantation; Immediate, immediate transplantation without EVLP; $P N$, recipient pneumonectomy. ${ }^{*} \mathrm{p}=0.01$ versus $\mathrm{PN}$.

\section{Histopathologic Lung Injury}

Histological differences were observed between EVLP and EVLP+Cold groups compared with Immediate and PN groups (Figure 8). When lung injury severity scores were quantified, a trend towards less injury was identified in groups EVLP and EVLP+Cold compared with Immediate (EVLP: $3.2 \pm 0.8$, EVLP+Cold: $3.4 \pm 0.9$ versus Immediate: $4.4 \pm 0.5, \mathrm{p}>0.05$ ).

Recipient PN lung (1.8 \pm 0.9$)$ was used as negative control. No significant differences in the components or in composite score of lung injury severity were observed between groups EVLP and EVLP+Cold. 


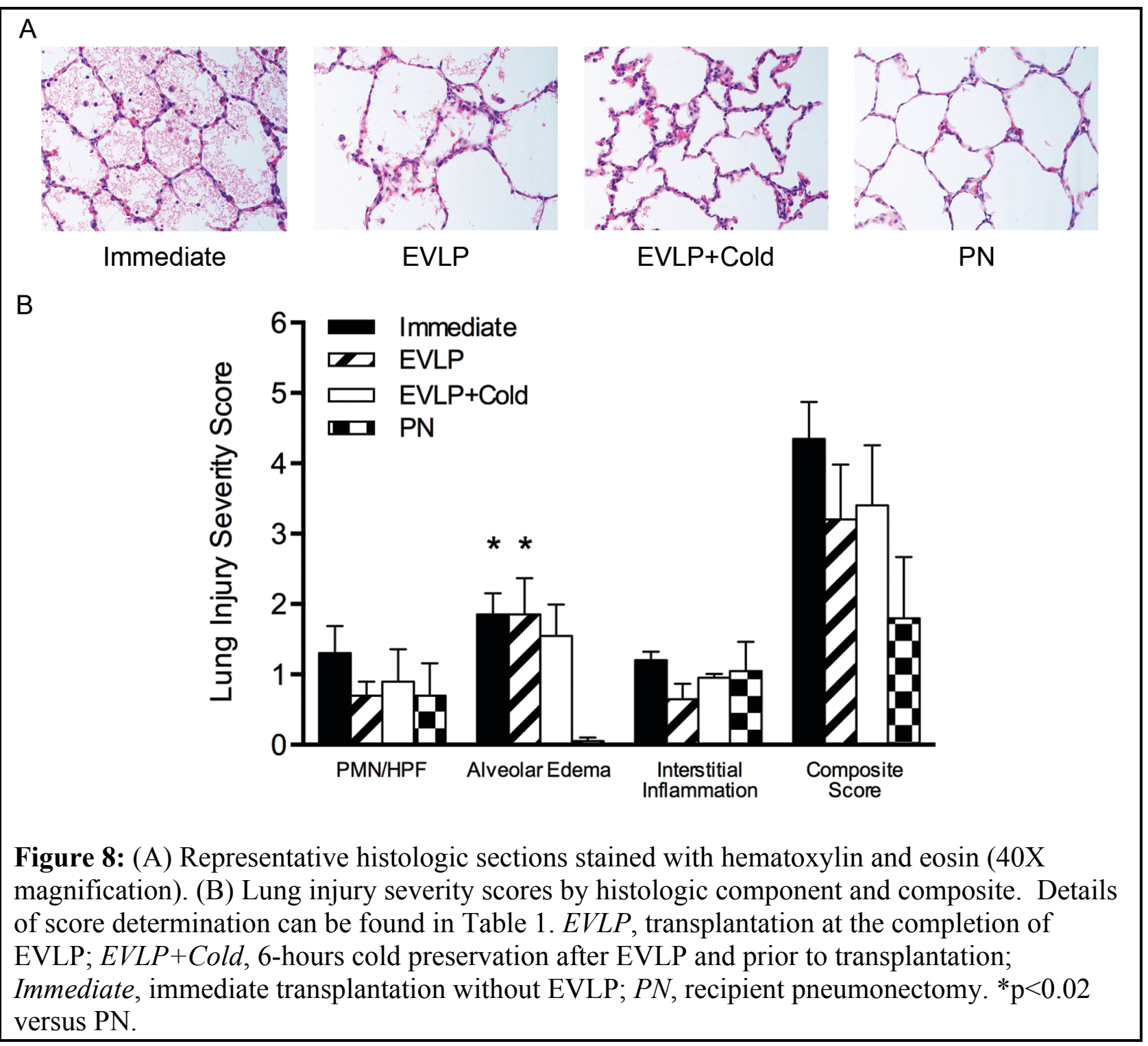

Significantly fewer neutrophils were observed in groups EVLP and PN compared with Immediate (EVLP: $39.6 \pm 5.6, \mathrm{PN}: 37.3 \pm 6.9$ versus Immediate: $103.4 \pm 18.5$, $<<0.01$ ) (Figure 9). No significant difference in the number of neutrophils per high-powered field was observed

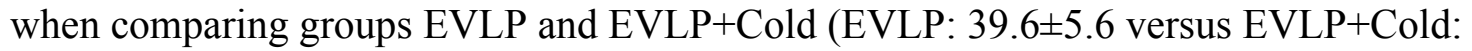
$62.3 \pm 10.7, \mathrm{p}>0.5)$. 


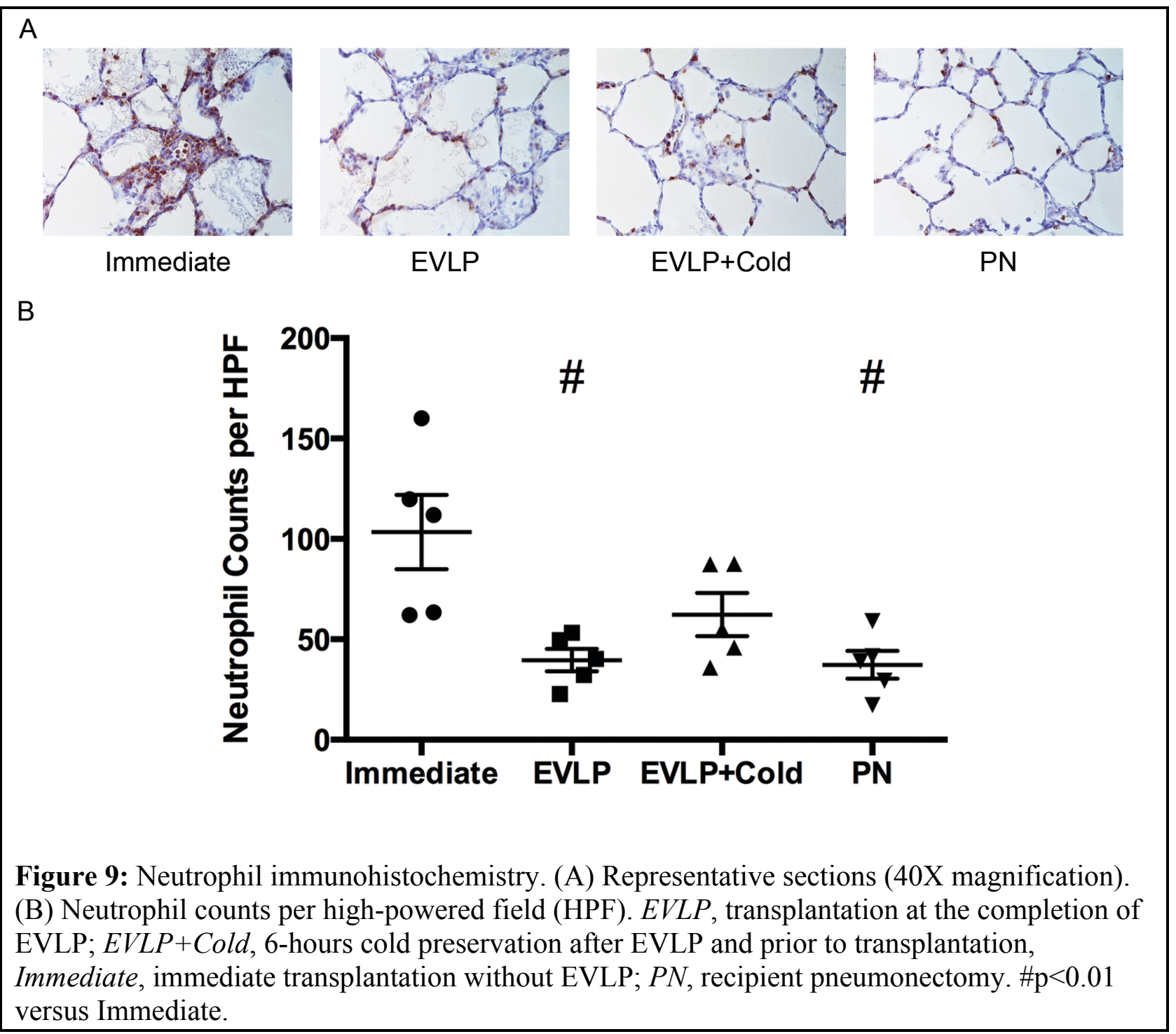

\section{Discussion}

The present study used a porcine model of DCD lung transplantation to evaluate the effect of prolonged cold preservation after EVLP on ischemia-reperfusion (IR) injury and graft function. The results demonstrate lung performance after transplantation of organs subjected to an additional 6-hours of cold ischemia time after EVLP is not inferior to lungs that are transplanted immediately after completion of EVLP. Additionally, both EVLP treatment arms of the study resulted in superior lung function compared with immediate transplantation of DCD 
lungs (control). These results were observed in both lung function (oxygenation and dynamic compliance) and in post-reperfusion analyses (tissue inflammatory cytokine expression and infiltration of neutrophils).

Current transplant centers utilizing EVLP have adopted either organ procurement with EVLP performed at the hospital of the recipient or early-initiation of mobile EVLP at the donor hospital. [35] Considering that not all transplants centers have the support necessary to perform EVLP, the current study identifies a role for cold preservation after EVLP, which may allow rehabilitated lungs to be shipped to appropriate recipients without additional graft deterioration. Lungs are often rejected for procurement due to imperfect donor characteristics (oxygenation capacity, chest radiograph findings, DCD status) or lack of an appropriate local recipient. The ultimate goal is to decrease wait list times and improve outcomes after transplantation. Encouraging more aggressive lung procurement (including DCD donors), allowing the determination of transplant suitability to occur during EVLP, and delivering rehabilitated lungs to the most appropriate recipient, will maximize the number of lungs transplanted.

This strategy of remote EVLP rehabilitation was clinically successful as described in a case report published by Wigfield $\mathrm{CH}$ and colleagues. [49] Our findings support this concept of "Regional Organ Assessment and Repair Centers," where strategically located EVLP centers function as the hub for organ assessment, rehabilitation, and distribution. [50] Increasing procurement rates will also increase the number of human lungs available for research, as not all lungs will improve to a level suitable for transplantation. This influx of human lungs into research will help expedite advances in using EVLP as a rehabilitative platform for gene therapy and targeted drug delivery. 
The results of the current study, using a DCD model of lung transplantation, suggest that an additional 6-hours of cold preservation after EVLP does not worsen the resultant IR injury. Most notably there were no significant differences in the levels of inflammatory cytokines between groups EVLP and EVLP+Cold. Additionally, the amount of pulmonary edema after reperfusion, the number of infiltrating neutrophils, and the extent of lung injury present on histologic examination were not different between groups EVLP and EVLP+Cold. The effect of hypothermia on lung tissue upon completion of EVLP involves many mechanisms, including decreasing infiltration of neutrophils, lowering cellular metabolism, and inhibition of apoptosis. [51]

Previous work in our lab identified the benefit of hypothermia prior to EVLP on subsequent lung function after transplantation [38] and the current study supports the addition of hypothermic storage after EVLP, if necessary, to increase lung utilization. Our findings using DCD donors support work published on the effects of prolonged cold preservation after EVLP using a heart-beating porcine model of transplantation. [52] The model used in the present study was previously used in our lab to assess the effect of EVLP after 12-hours cold preservation of DCD porcine lungs. [40] Additionally, our lab has shown that EVLP can rehabilitate DCD lungs exposed to 60-minutes of warm ischemia in a preclinical porcine model. [38] These DCD models used in our lab complement studies assessing the benefits of EVLP using heart-beating models of lung transplantation.

The current study is limited by the relatively short period of warm ischemia after circulatory death and does not account for the compounding effects of cold ischemia time after EVLP with prolonged warm ischemia time prior to donor lung procurement. Additionally, the findings from this large animal model are limited by the natural heterogeneity between animals 
and the low sample size per group. Measuring indicators of IR injury after 2 hours of in vivo reperfusion also limit the conclusions and may be more meaningful if assessed after 12-24 hours. Further investigation into the molecular effects of cold ischemia after normothermic EVLP is needed to better understand the long-term consequences on graft function and survival.

The findings of this study support future research directed towards identifying the limits of pre- and post-EVLP cold preservation. The 6-hour window supported by this study is sufficient to deliver lungs almost anywhere in the U.S., but extending that period of time may still be helpful. Further understanding of how to determine lung suitability for transplantation during EVLP will allow an appropriate cold preservation time after EVLP to be assigned accordingly to each set of lungs. Eventually a comprehensive algorithm accounting for preEVLP characteristics (DCD status, ischemia time), indicators of performance and rehabilitation while on EVLP, and the necessary post-EVLP transport time, will help maximize donor lung utilization.

In conclusion, the addition of 6-hours of cold preservation after EVLP does not result in significant differences compared to lungs transplanted immediately following EVLP. This study demonstrates EVLP followed by cold preservation may be an effective strategy for improving organ utilization through the development of lung rehabilitation centers. The current mismatch between lung transplant candidates awaiting a suitable organ and the number of acceptable donor organs procured and allocated may be alleviated with adoption of this approach. Expanding the donor pool and optimizing lung utilization may lead to an improvement in wait list mortality and increase the rate of successful lung transplantation. 


\title{
PROJECT \#2
}

\section{Ex Vivo Assessment of Donation after Circulatory Death Lungs Exposed to Increasing Warm Ischemia Time}

\begin{abstract}
Eric J Charles MD ${ }^{1}$ J. Hunter Mehaffey MD${ }^{1}$, Mary E Huerter MD MA Ashish K Sharma MBBS $\mathrm{PhD}^{1}$, Yunge Zhao MD PhD ${ }^{1}$, Mark H Stoler MD², James M Isbell MD MSCI ${ }^{1}$, Mark E Roeser MD¹, Dustin M. Walters MD,
\end{abstract} Christine L Lau MD MBA ${ }^{1}$, Curtis G Tribble MD ${ }^{1}$, Irving L Kron MD ${ }^{1}$, Victor E Laubach PhD $^{1}$

Departments of ${ }^{1}$ Surgery and ${ }^{2}$ Pathology, University of Virginia, Charlottesville, Virginia

Status of Research: MANUSCRIPT IN PREPARATION 


\section{Abstract}

Background: Increased utilization of donation after circulatory death (DCD) lungs may help alleviate the supply/demand mismatch between available donor organs and lung transplant candidates.

Methods: Using an established porcine DCD model, we sought to determine the effect of increasing warm ischemia time after circulatory arrest on lung function during ex vivo lung perfusion (EVLP). Donors underwent hypoxic cardiac arrest, followed by 60, 90, or 120 minutes of warm ischemia time (WIT) prior to procurement and 4 hours of EVLP. Oxygenation, pulmonary artery and airway pressures, and compliance were measured hourly. Lung injury scores and neutrophil counts were assessed histologically.

Results: After EVLP, all three groups met all the criteria for transplantation, except for 90minute WIT lungs, which had a mean PA pressure increase greater than $15 \%$. There were no significant differences between groups as assessed by final oxygenation capacity, as well as change in PA pressure, airway pressure, and compliance. There were also no differences in lung injury scores or neutrophil counts.

Conclusions: These results suggest that longer WIT alone (within 60-120 min) does not predict worse lung function at the conclusion of EVLP. Expanding acceptable WIT after circulatory death may eventually allow for inclusion of uncontrolled DCD lungs in procurement protocols. 


\section{Introduction}

Over the past 10 years, lung transplant volume in the United States increased by $66 \%$, from 1405 transplants in 2006, to 2327 transplants in 2016. Unfortunately, wait list additions have increased at a similar rate, with 2,789 new registrations in 2016. [2] Various centers are now performing transplants using Maastricht category III controlled donation after circulatory death (DCD) lungs, in an effort to maximize the number of transplants performed. [53] As the supply of traditional donation after brain death (DBD) lungs remains stagnant and wait list mortality continues to rise, DCD lungs have become an important source of additional organs. [3] Studies to date suggest that outcomes with Maastricht category III DCD lungs are similar to outcomes with DBD lungs. [8-10] Even with inclusion of DCD lungs in transplant protocols, overall utilization remains low, and the supply of acceptable organs has not fulfilled the everincreasing demand. $[11,12]$

Uncontrolled DCD lungs (Maastricht categories I and II) are an additional source of available organs but are currently not utilized for transplantation in the United States. [11, 54] There are several reasons for this, including ethical concerns, logistical barriers, and graft function uncertainty. Warm ischemia time (WIT) varies greatly depending on whether the patient dies in the hospital or out in the community, and whether or not adequate, timely cardiopulmonary resuscitation is performed. With the availability of ex vivo lung perfusion (EVLP) as a platform for graft assessment and potential rehabilitation, uncontrolled DCD lungs may one day become a valuable source of transplantable organs. [11]

Our laboratory has experience studying DCD lung transplantation using a porcine model of hypoxic cardiac arrest. [38] Using this model, we have evaluated the effect of cold ischemia time before and after EVLP, ventilation strategies during EVLP, and ex vivo lung rehabilitation 
with adenosine pathway modulation. [55-58] Building on our previous work, the objective of this study was to determine the effect of increasing WIT after circulatory arrest on lung function during EVLP. We anticipate that EVLP will be standard practice for the assessment of uncontrolled DCD lungs prior to transplantation allowing use of these lungs to become a reality. Therefore, we sought to understand how differences in WIT affect commonly used lung function parameters for determining whether or not to transplant lungs at the conclusion of EVLP. We hypothesized that DCD lungs exposed to increasing WIT up to 120 minutes after circulatory arrest would still meet transplant criteria after 4 hours of normothermic EVLP.

\section{Materials and Methods}

All animals received humane care in compliance with the 2011 Guide for the Care and Use of Laboratory Animals, $8^{\text {th }}$ edition as recommended by the US National Institutes of Health. The University of Virginia Animal Care and Use Committee approved this study.

\section{Animals and Hypoxic Cardiac Arrest Procedure}

Mature domestic swine of both sexes $(27-34 \mathrm{~kg})$ were used. Animals were anesthetized (intramuscular injection: $6 \mathrm{mg} / \mathrm{kg}$ tiletamine/zolzazepam, $2 \mathrm{mg} / \mathrm{kg}$ xylazine), intubated, weighed, and ventilated ( $3 \%$ isoflurane, tidal volume $8 \mathrm{~mL} / \mathrm{kg}$, respiratory rate $18-20$ breaths $/ \mathrm{min}$, positive end-expiratory pressure [PEEP] $5.0 \mathrm{cmH}_{2} \mathrm{O}$, and 1.0 fraction of inspired oxygen $\left[\mathrm{FiO}_{2}\right]$ ), as

previously described. [45] Systemic heparin was not administered. A baseline arterial blood gas (ABG) sample was obtained and continuous electrocardiogram monitoring initiated. The endotracheal tube was clamped mid-inspiration to initiate hypoxia. Every 1-2 minutes thereafter, an $\mathrm{ABG}$ sample was obtained to characterize the physiologic changes taking place during 
hypoxia. $\mathrm{pH}$, partial pressure of arterial carbon dioxide $\left(\mathrm{PaCO}_{2}\right)$, partial pressure of arterial oxygen $\left(\mathrm{PaO}_{2}\right)$, bicarbonate level $\left(\mathrm{HCO}_{3}\right)$, base excess, and lactic acid concentration were recorded with each sample. Mean arterial pressure (MAP) and heart rate were recorded continuously until asystole, at which point the WIT began.

\section{Randomization}

After hypoxic cardiac arrest, animals were randomized to 60 (60 WIT, n=3), 90 (90 WIT, $\mathrm{n}=6)$, or 120 (120 WIT, $\mathrm{n}=6)$ min of warm ischemia prior to standard lung procurement. Subsequently, all lungs underwent 4 hours of normothermic EVLP according to the Toronto protocol. [47] Exclusion criteria according to the Toronto protocol were used in this study to determine transplant suitability (partial pressure of oxygen to fraction of inspired oxygen ratio $\left[\mathrm{PO}_{2} / \mathrm{FiO}_{2}\right]<400 \mathrm{mmHg}$, greater than $15 \%$ increase of pulmonary artery $[\mathrm{PA}]$ pressure, and greater than 15\% deterioration of airway pressures and compliance). [59] Group 60 WIT served as the control arm of the study based on previous data from our laboratory showing reasonable $e x$ vivo lung function after hypoxic cardiac arrest and 60 min of warm ischemia. [38]

\section{Donor Lung Procurement}

At completion of the prescribed WIT, ventilation was resumed at the pre-arrest settings. The thorax was accessed via median sternotomy, the pericardium and pleura were incised, and the main PA was cannulated with a cardioplegia cannula (Terumo Heart, Ann Arbor, MI). Prostaglandin-E1 (500 $\mu \mathrm{g}$, Pfizer, New York, NY) followed by $1.5 \mathrm{~L}$ of $4^{\circ} \mathrm{C}$ Perfadex (XVIVO Perfusion, Englewood, CO) with 15,000 IU heparin (Hospira Inc., Lake Forest, IL) was flushed through the lungs and drained via left atrial (LA) appendage atriotomy. The venae cavae were 
ligated and the lungs were cooled with topical ice slush. After completion of the flush, the trachea was clamped mid-inspiration and the heart-lung bloc procured.

\section{Lung Preparation and Initiation of EVLP}

After back-table removal of the heart and preparation of the trachea, main PA, and LA cuff, an endotracheal (ET) tube and yellow and green cannulas (XVIVO Perfusion, Englewood, CO) were sutured into place to set up a closed atrial system, as described by the Toronto group. $[46,47]$ The lungs were maintained cold on a bed of ice during preparation. A retrograde flush was performed with $500 \mathrm{~mL}$ of $4^{\circ} \mathrm{C}$ Perfadex prior to placing the lungs on the EVLP circuit. The target cold ischemic period from end of WIT to initiation of EVLP was 1 hour.

EVLP was performed as previously described. [38] The circuit was primed with $2 \mathrm{~L}$ of acellular Steen Solution (XVIVO Perfusion, Englewood, CO) at room temperature, supplemented with $500 \mathrm{mg}$ cefazolin (APP Pharmaceuticals, Schaumburg, IL), $500 \mathrm{mg}$ methylprednisolone (Pfizer, New York, NY), and 10,000 IU heparin. [47, 59] The lungs were transferred to the EVLP chamber and flow was initiated through the LA cannula to remove air from the pulmonary vasculature and PA cannula. The inflow tubing was then connected to the PA cannula and forward flow was initiated at $0.2 \mathrm{~L} / \mathrm{min}$. Over the first 30 minutes, the perfusate was warmed to $37^{\circ} \mathrm{C}$ using a heat exchanger. At $32^{\circ} \mathrm{C}$, ventilation was initiated (tidal volume 8 $\mathrm{mL} / \mathrm{kg}$, respiratory rate 8 breaths/min, PEEP $5.0 \mathrm{cmH}_{2} \mathrm{O}, \mathrm{FiO}_{2} 0.21$ ). Over the subsequent 30 minutes, flow was increased to a target rate of $40 \%$ of estimated cardiac output $(100 \mathrm{~mL} / \mathrm{kg}$ donor body weight). A tri-gas mixture ( $86 \%$ nitrogen, $8 \%$ carbon dioxide, $6 \%$ oxygen) was used to de-oxygenate the perfusate with a target inflow partial pressure of carbon dioxide $\left(\mathrm{PCO}_{2}\right)$ of 
$35-45 \mathrm{mmHg}$. LA pressures were maintained between 2 and $5 \mathrm{mmHg}$ by adjusting the height of the perfusate reservoir.

\section{Lung Function Assessment}

EVLP was run continuously for 4 hours. At each hour, recruitment maneuvers were performed and the $\mathrm{FiO}_{2}$ was increased to 1.0 for 15 minutes. Inflow (PA) and outflow (LA) perfusate samples were obtained to measure the functional partial pressure of oxygen $\left(\mathrm{PO}_{2}\right)$. Peak and plateau airway pressures $\left(\mathrm{cm} \mathrm{H}_{2} \mathrm{O}\right)$ were recorded and used to calculate dynamic and static compliance $\left(\mathrm{mL} / \mathrm{cm} \mathrm{H}_{2} \mathrm{O}\right)$. After 4 hours, the lungs were removed from the circuit and flushed antegrade with $500 \mathrm{~mL}$ of $4^{\circ} \mathrm{C}$ Perfadex.

\section{Pulmonary Edema Assessment}

Three samples of fresh tissue (from the same anatomic locations on each set of lungs) were obtained at the end of EVLP and weighed individually. Following desiccation in a vacuum oven, dry tissue weights were obtained and wet-to-dry weight ratios calculated. For each set of lungs, all three ratios were averaged together to obtain one wet-to-dry weight ratio per set of lungs that assessed overall edema accumulation.

\section{Histology}

The lower lobe of each left lung was fixed in formalin overnight. Four tissue samples per lung were obtained, paraffin-embedded, and sectioned. One section from each sample was stained with hematoxylin-eosin (H\&E) and used to calculate an average lung injury severity score. A second section from each sample was stained to quantify neutrophil numbers by 
immunohistochemistry analysis. H\&E sections were evaluated by a masked pathologist and lung injury severity scores were based on polymorphonuclear cells per 40X high-powered field (HPF) $(0=<5,1=6-10,2=11-20,3=>20)$, alveolar edema $(0=<5 \%, 1=6-25 \%, 2=26-50 \%, 3=>50 \%)$, and interstitial inflammation $(0=$ none, $1=$ minimal, $2=$ moderate, $3=$ severe $)$, as previously described. [60] For neutrophil immunohistochemistry, mouse monoclonal anti-porcine neutrophil antibody (MBA Biomedicals, Augst, Switzerland) was used, followed by donkey anti-mouse IgG (Jackson ImmunoResearch Laboratories Inc., West Grove, PA). [48] Slides were incubated with 3,3diaminobenzidine tetrahydrochloride (Dako Inc., Carpinteria, CA) to produce brown precipitate and counterstained with hematoxylin. Neutrophils in five 40X microscopic photographs per slide were counted by a masked reviewer and averaged.

\section{Statistical Analysis}

Statistical comparisons between groups were conducted using one-way analysis of variance with Bonferroni's correction for multiple comparisons or Student's t-test depending on the number of groups in each analysis. Data are reported as mean \pm standard error of the mean, with p-value for significance of 0.05 . Linear and nonlinear (exponential, sigmoidal, and polynomial) regression were used to generate best-fit lines with $95 \%$ confidence interval bands for data shown in Figures 10 and 11. All four models were compared with goodness of fit testing used to determine which model best represented each dataset. All statistical analyses were performed using Prism 7 (GraphPad Software, La Jolla, CA). 


\section{Results}

Donor Animal Characteristics and Elapsed Time

There were no significant differences between groups in terms of donor animal weight, baseline $\mathrm{PaO}_{2} / \mathrm{FiO}_{2}$, or elapsed time from ET tube clamp to asystole, with a mean weight for all animals of $30 \pm 0.7 \mathrm{~kg}$, baseline $\mathrm{PaO}_{2} / \mathrm{FiO}_{2}$ of $494 \pm 22 \mathrm{mmHg}$, and time from clamp to asystole of 22.2 $\pm 1.7 \mathrm{~min}$. Donor animal characteristics and the elapsed time at each stage of the experiment are shown in Table 2. Since groups were randomized based on duration of WIT, there were significant differences in time from ET tube clamp to cold flush (60 WIT: $80 \pm 0$ vs. 90 WIT: 114.5 \pm 3.4 vs. 120 WIT: $141 \pm 2.6 \mathrm{~min}, \mathrm{p}<0.0001)$, time from ET tube clamp to initiation of EVLP (60 WIT: $143.7 \pm 1.3$ vs. 90 WIT: $179.3 \pm 4.0$ vs. 120 WIT: $203.8 \pm 6.1 \mathrm{~min}, \mathrm{p}<0.0001$ ), and total elapsed time from ET tube clamp to completion of EVLP (60 WIT: $383.7 \pm 1.3$ vs. 90 WIT:

$419.3 \pm 4.0$ vs. 120 WIT: $443.8 \pm 6.1 \mathrm{~min}, \mathrm{p}<0.0001)$.

Table 2: Donor animal characteristics and elapsed time during experiment

\begin{tabular}{|c|c|c|c|c|c|}
\hline & $\begin{array}{c}60 \text { WIT } \\
(n=3)\end{array}$ & $\begin{array}{c}90 \text { WIT } \\
(n=6)\end{array}$ & $\begin{array}{c}120 \text { WIT } \\
(\mathrm{n}=6)\end{array}$ & p-value & $\begin{array}{l}\text { All Animals } \\
(n=15)\end{array}$ \\
\hline \multicolumn{6}{|l|}{ Donor characteristics } \\
\hline Animal weight (kg) & $29.2 \pm 1.2$ & $29.8 \pm 1.0$ & $30.6 \pm 1.5$ & 0.78 & $30.0 \pm 0.7$ \\
\hline Initial $\mathrm{PaO} 2 / \mathrm{FiO} 2$ & $494 \pm 54$ & $509 \pm 23$ & $481 \pm 46$ & 0.87 & $494 \pm 22$ \\
\hline \multicolumn{6}{|l|}{ Elapsed time (min) } \\
\hline ET tube clamp to asystole & $20 \pm 0$ & $24.5 \pm 3.4$ & $21.0 \pm 2.6$ & 0.57 & $22.2 \pm 1.7$ \\
\hline Warm ischemia & 60 & 90 & 120 & - & - \\
\hline ET tube clamp to cold flush & $80 \pm 0$ & $114.5 \pm 3.4$ & $141 \pm 2.6$ & $<0.0001$ & - \\
\hline \multicolumn{6}{|l|}{ Cold ischemia (min) } \\
\hline Cold flush to lungs out & $15 \pm 0.6$ & $19.8 \pm 1.3$ & $18.8 \pm 1.2$ & 0.10 & $18.5 \pm 0.8$ \\
\hline Lungs out to EVLP start & $48.7 \pm 1.9$ & $45 \pm 3.1$ & $44 \pm 5.1$ & 0.78 & $45.3 \pm 2.3$ \\
\hline Total cold ischemia & $63.7 \pm 1.3$ & $64.8 \pm 3.9$ & $62.8 \pm 4.5$ & 0.94 & $63.8 \pm 2.3$ \\
\hline Time prior to EVLP (min) & $143.7 \pm 1.3$ & $179.3 \pm 4.0$ & $203.8 \pm 6.1$ & $<0.0001$ & - \\
\hline Normothermic EVLP (min) & 240 & 240 & 240 & - & 240 \\
\hline Total elapsed time (min) & $383.7 \pm 1.3$ & $419.3 \pm 4.0$ & $443.8 \pm 6.1$ & $<0.0001$ & - \\
\hline
\end{tabular}

Groups compared using one-way analysis of variance. Mean \pm standard error of the mean reported. ET: endotracheal; EVLP: ex vivo lung perfusion; PaO2/FiO2: ratio of partial pressure of oxygen to fraction of inspired oxygen. 


\section{Physiologic Changes During Hypoxia}

ABG samples were obtained every 1-2 min during hypoxia. Changes over time from ET tube clamp to asystole for each parameter are shown in Figure 10. Changes in MAP and heart rate are shown in Figure 11. The normal range is indicated with horizontal shading to identify the time at which the parameter became abnormal. The mean time of death was $22.2 \mathrm{~min}$ (vertical line, Figures 10 and 11), which displays how abnormal each parameter was on average upon initiation of the prescribed warm ischemia period. Within 3 min of inducing hypoxia, $\mathrm{pH}$, $\mathrm{PaCO}_{2}, \mathrm{HCO}_{3}$, and base excess were all abnormal. After 6 min, MAP and lactic acid levels were abnormal, and within 8 min, $\mathrm{PO}_{2}$ values fell below the normal range. Heart rate remained within the normal range until 14 min on average. 
A.

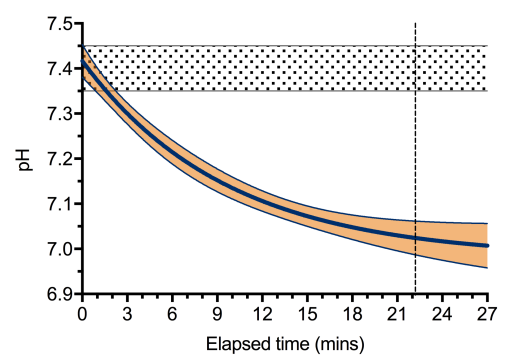

D.

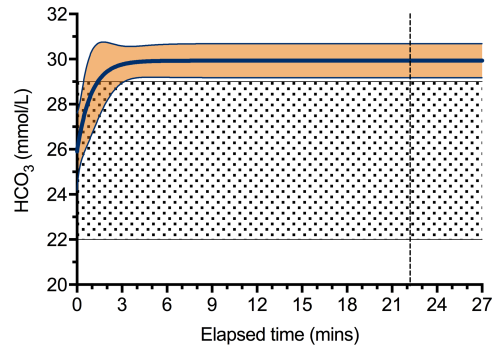

B.

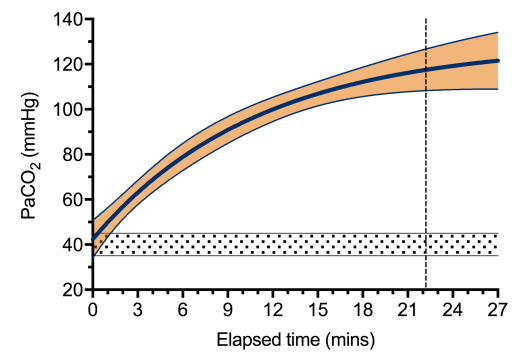

E.

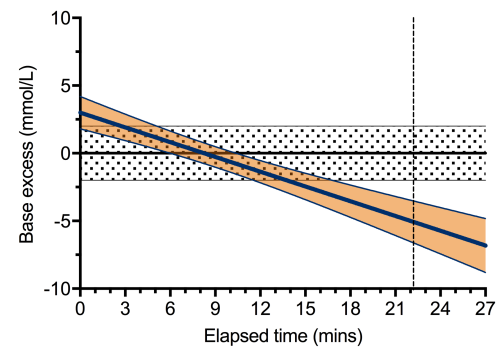

C.

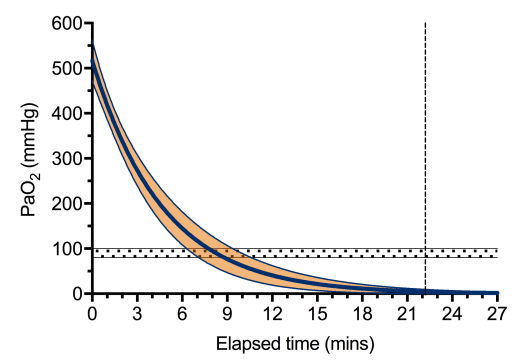

F.

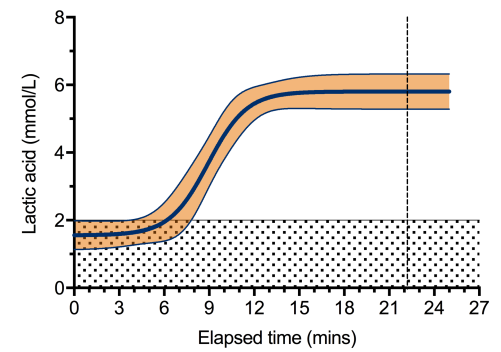

Figure 10: Physiologic changes during hypoxia from endotracheal tube clamp to circulatory arrest. The horizontal shading indicates normal ranges for each parameter. The vertical dashed line indicates the mean time of death (22.2 min). (A) pH. (B) Partial pressure of arterial carbon dioxide $\left(\mathrm{PaCO}_{2}\right)$. (C) Partial pressure of arterial oxygen $\left(\mathrm{PO}_{2}\right)$. (D) Bicarbonate level $\left(\mathrm{HCO}_{3}\right)$. (E) Base excess. (F) Lactic acid level.

A.

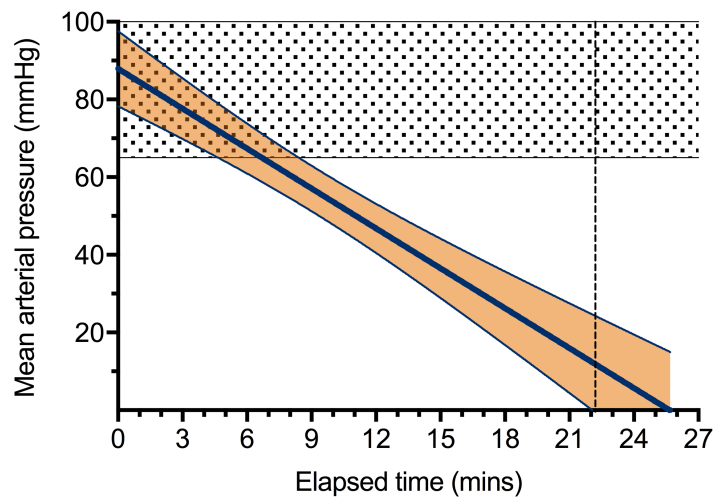

B.

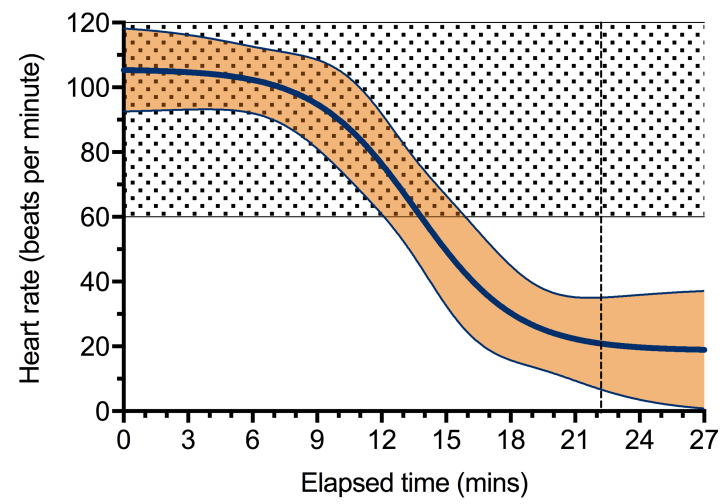

Figure 11: Mean arterial pressure (A) and heart rate (B) during hypoxia from endotracheal tube clamp to circulatory arrest. The horizontal shading indicates normal ranges for each parameter. The vertical dashed line indicates the mean time of death (22.2 min). 


\section{Oxygenation Capacity and PA Pressures During EVLP}

Mean $\mathrm{PO}_{2} / \mathrm{FiO}_{2}$ at each hour during EVLP is shown in Figure 12A. Final $\mathrm{PO}_{2} / \mathrm{FiO}_{2}$ after 4 hours of EVLP was not significantly different between groups (60 WIT: $518.3 \pm 33.7$ vs. 90 WIT: $477.2 \pm 31.7$ vs. $120 \mathrm{WIT}: 427 \pm 36.2 \mathrm{mmHg}, \mathrm{p}=0.28$ ), with all three meeting the oxygenation threshold for transplant suitability (final $\mathrm{PO}_{2} / \mathrm{FiO}_{2}>400 \mathrm{mmHg}$ ). [59] Oxygenation capacity increased during EVLP for all groups, with no significant difference between groups in percent change of $\mathrm{PO}_{2} / \mathrm{FiO}_{2}$ (Figure 12B). EVLP significantly improved the oxygenation capacity of 60 WIT ( $\mathrm{p}=0.01$ ) and 90 WIT ( $\mathrm{p}=0.01$ ) lungs, but did not for 120 WIT lungs $(\mathrm{p}=0.6$ ) (Figure 12C). The $\mathrm{PA}^{\mathrm{PCO}_{2}}$ levels were within the target range of $35-45 \mathrm{mmHg}$ for all animals, with no difference between groups (60 WIT: $41.1 \pm 0.9$ vs. 90 WIT: $40.7 \pm 0.5$ vs. 120 WIT: $42.1 \pm 0.5 \mathrm{mmHg}, \mathrm{p}=0.15)$.

Mean PA pressures during EVLP were different between groups at hours $1(\mathrm{p}=0.02)$ and $2(\mathrm{p}=0.04)$, but not at the completion of EVLP ( 60 WIT: $18.7 \pm 1.7$ vs. 90 WIT: $18.7 \pm 0.9$ vs. 120 WIT: $22 \pm 1.3 \mathrm{mmHg}, \mathrm{p}=0.12$ ) (Figure 12D). PA pressure increased during EVLP for all three groups. The percent change remained below the exclusion criteria of $15 \%$ for groups 60 WIT $(14 \pm 7.7 \%)$ and 120 WIT $(7.7 \pm 6.4 \%)$, but not for group 90 WIT $(30.8 \pm 5.0 \%, \mathrm{p}=0.04)$ (Figure 12E). Final PA pressures were significantly higher compared to starting PA pressures for group 90 WIT $(\mathrm{p}=0.001)$ (Figure 12F). LA pressures were maintained within the target range for all groups (60 WIT: $2.3 \pm 0.5$ vs. 90 WIT: $2.5 \pm 0.3$ vs. 120 WIT: $2.8 \pm 0.2 \mathrm{mmHg}, \mathrm{p}=0.5$ ). 


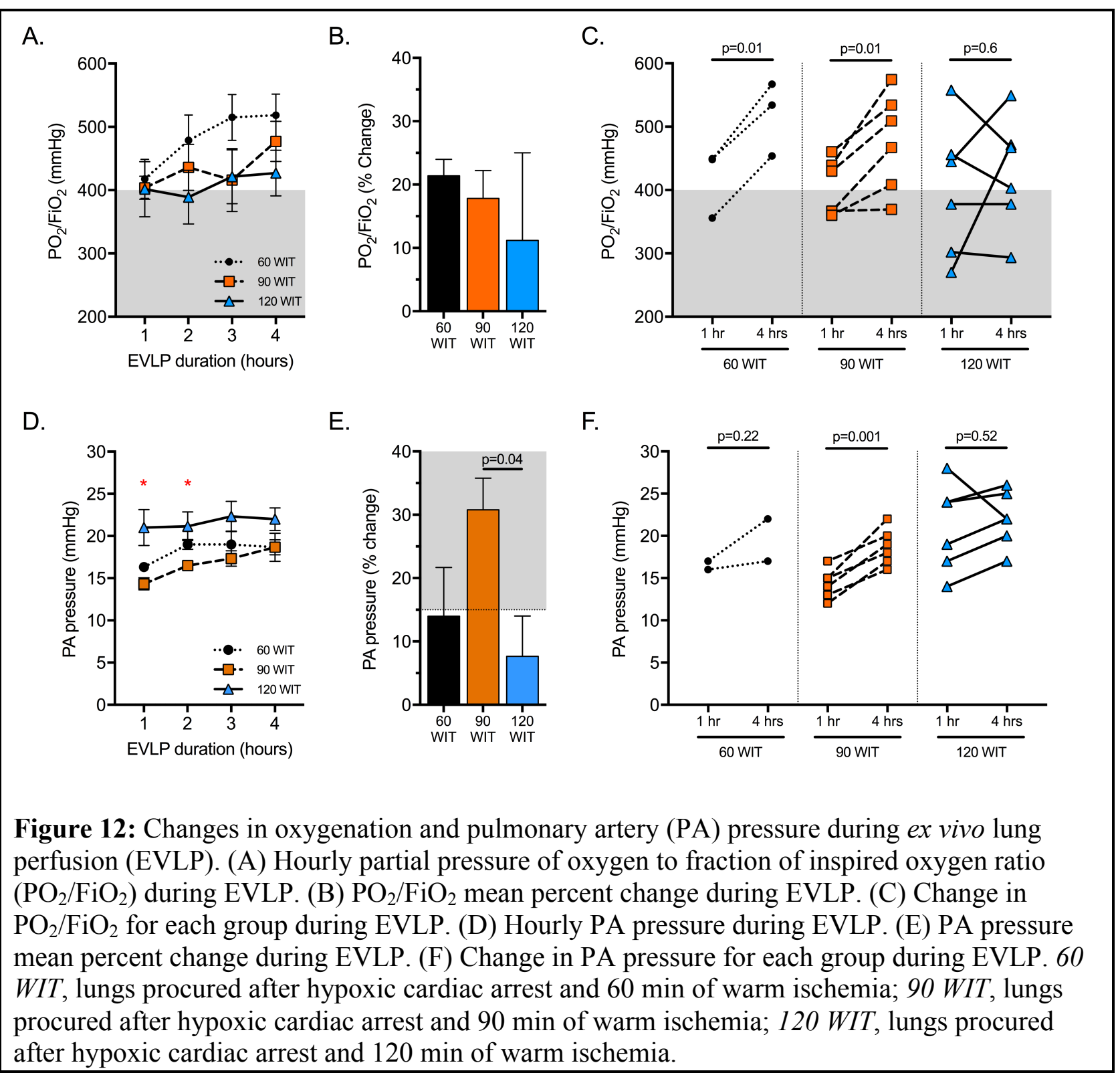

Plateau and Peak Airway Pressures During EVLP

There were no significant differences in plateau or peak airway pressures between groups at each hour of EVLP (Figures 13A, 13D). The percent change in airway pressures was also not different between groups, with all groups having mean changes in plateau and peak airway pressures that fell below the exclusion criteria of 15\% (Figures 13B, 13E). Final airway 
pressures were not significantly different from starting airway pressures in all three groups

\section{(Figures 13C, 13F).}

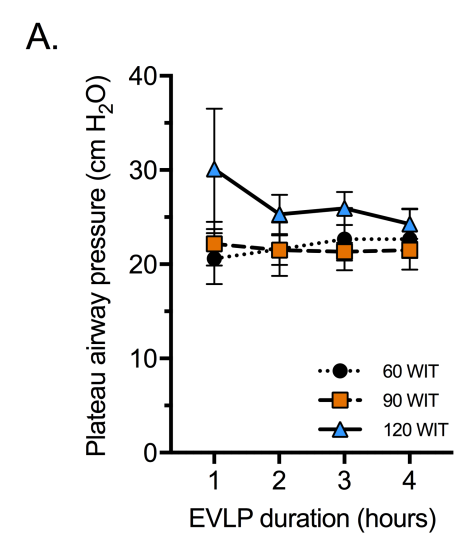

B.

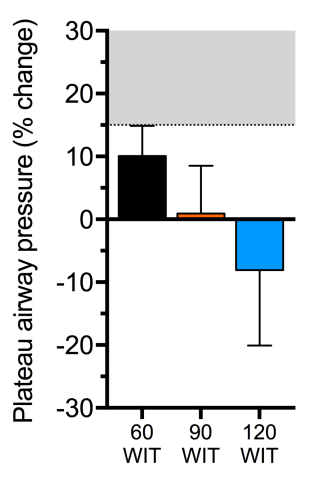

D.

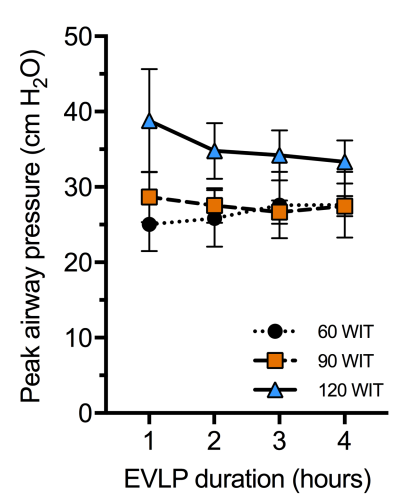

E.

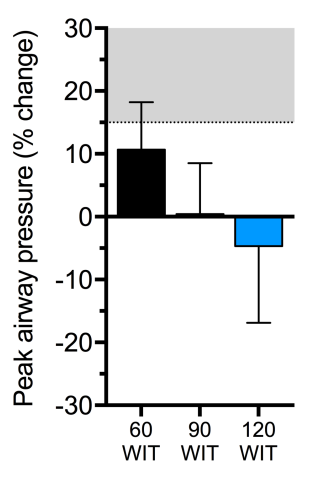

C.

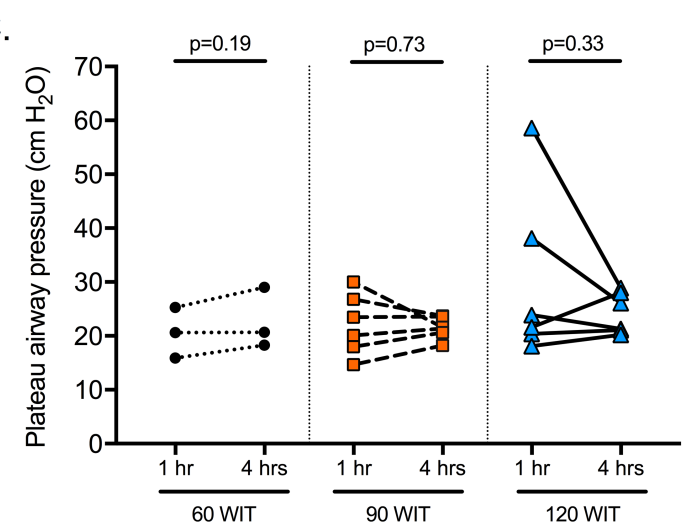

F.

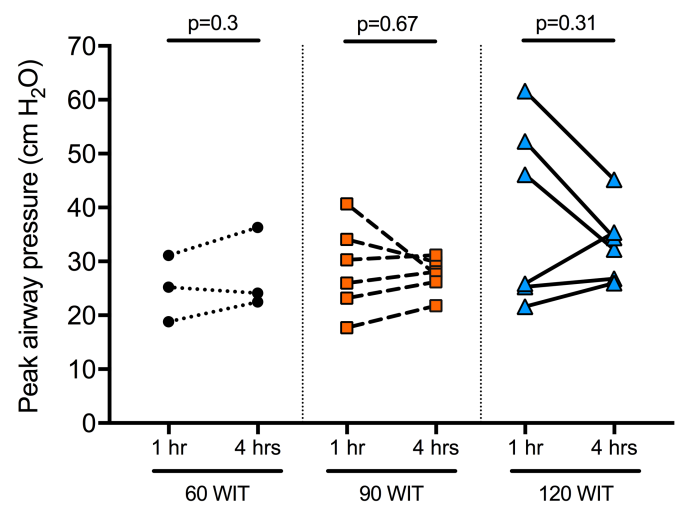

Figure 13: Changes in airway pressures during ex vivo lung perfusion (EVLP). (A) Hourly plateau airway pressures during ex vivo lung perfusion (EVLP). (B) Mean percent change in plateau airway pressure during EVLP. (C) Change in plateau airway pressures for each group during EVLP. (D) Hourly peak airway pressures during EVLP. (E) Mean percent change in peak airway pressure during EVLP. (F) Change in peak airway pressures for each group during EVLP. $60 \mathrm{WIT}$, lungs procured after hypoxic cardiac arrest and $60 \mathrm{~min}$ of warm ischemia; $90 \mathrm{WIT}$, lungs procured after hypoxic cardiac arrest and $90 \mathrm{~min}$ of warm ischemia; $120 \mathrm{WIT}$, lungs procured after hypoxic cardiac arrest and 120 min of warm ischemia.

\section{Static and Dynamic Compliance During EVLP}

Static and dynamic compliance at each hour of EVLP were not significantly different between groups (Figures 14A, 14D). Percent change in static compliance (60 WIT: $-11.4 \pm 5.4 \%$ vs. 90 WIT: $2.6 \pm 11.3 \%$ vs. 120 WIT: $25.2 \pm 23.2 \%, \mathrm{p}=0.43$ ) and dynamic compliance (60 WIT: - 
$10.7 \pm 8.3 \%$ vs. 90 WIT: $4.0 \pm 12.2 \%$ vs. 120 WIT: $15.6 \pm 16.3 \%, p=0.53$ ) were not significantly

different between groups, with all groups having mean changes within the acceptable limit for transplant suitability (less than $15 \%$ deterioration) (Figures 14B, 14E). EVLP did not significantly change static and dynamic compliance values for any group (Figures 14C, 14F).

A.

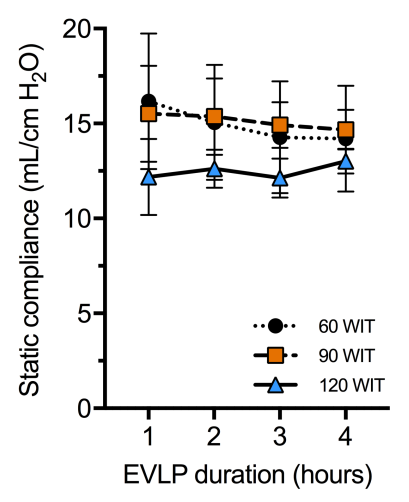

D.

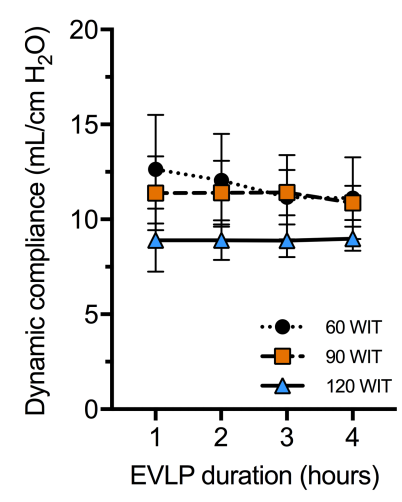

B.

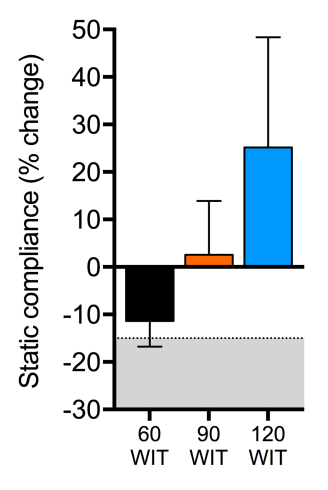

E.

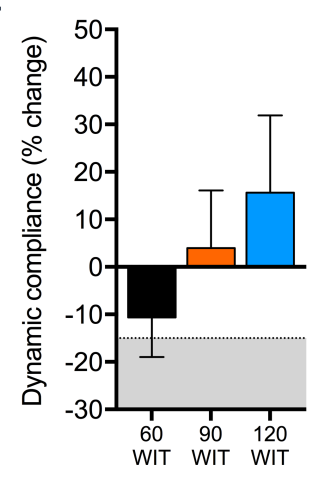

C.

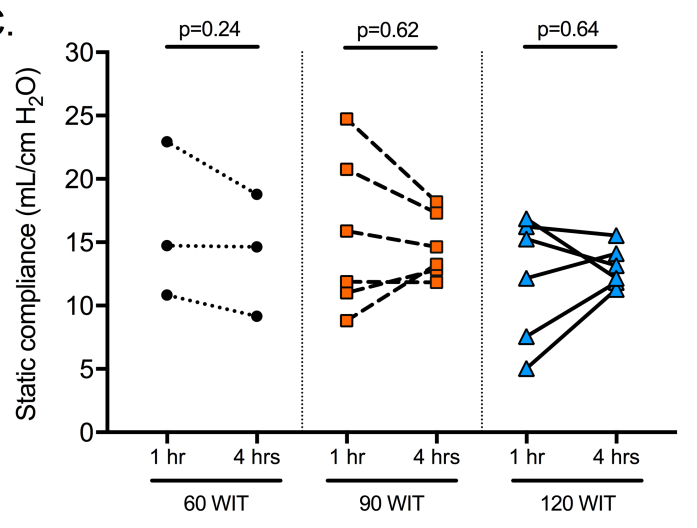

F.

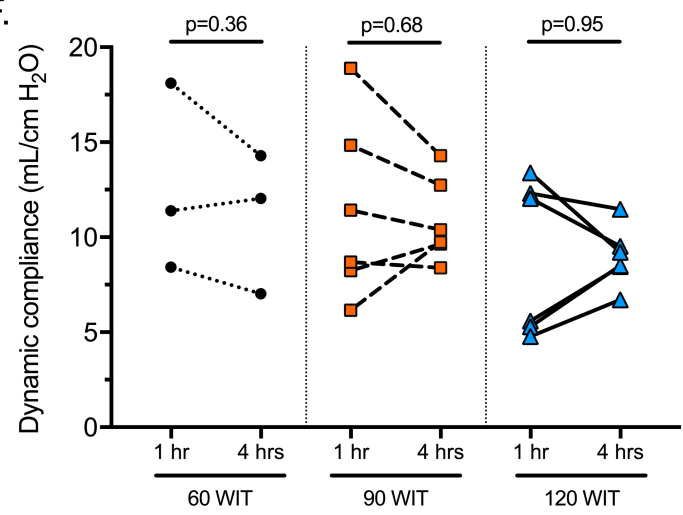

Figure 14: Changes in compliance during ex vivo lung perfusion (EVLP). (A) Hourly static compliance during ex vivo lung perfusion (EVLP). (B) Mean percent change in static compliance during EVLP. (C) Change in static compliance for each group during EVLP. (D) Hourly dynamic compliance during EVLP. (E) Mean percent change in dynamic compliance during EVLP. (F) Change in dynamic compliance for each group during EVLP. 60 WIT, lungs procured after hypoxic cardiac arrest and $60 \mathrm{~min}$ of warm ischemia; $90 \mathrm{WIT}$, lungs procured after hypoxic cardiac arrest and 90 min of warm ischemia; 120 WIT, lungs procured after hypoxic cardiac arrest and 120 min of warm ischemia. 
Pulmonary Edema after EVLP

Wet-to-dry weight ratios were calculated to assess pulmonary edema. There were no significant differences between groups in wet-to-dry weight ratios (60 WIT: $6.3 \pm 0.5$ vs. 90 WIT: $7.1 \pm 0.7$ vs. 120 WIT: $7.4 \pm 0.3, \mathrm{p}=0.36)$.

Histologic Assessment after EVLP

Composite lung injury severity scores were not significantly different between groups (60 WIT: $2.1 \pm 0.5$ vs. 90 WIT: $2.6 \pm 0.7$ vs. 120 WIT: $3.8 \pm 0.3, \mathrm{p}=0.14$ ) (Figures 15A, 15B), however lung injury severity scores did rise as WIT increased. Neutrophil numbers were also not significantly different between groups (60 WIT: $21.5 \pm 0.9$ vs. 90 WIT: $23.5 \pm 3.7$ vs. 120 WIT: 23.0 $\pm 8.0, \mathrm{p}=0.96)$ (Figures 15C, 15D). 

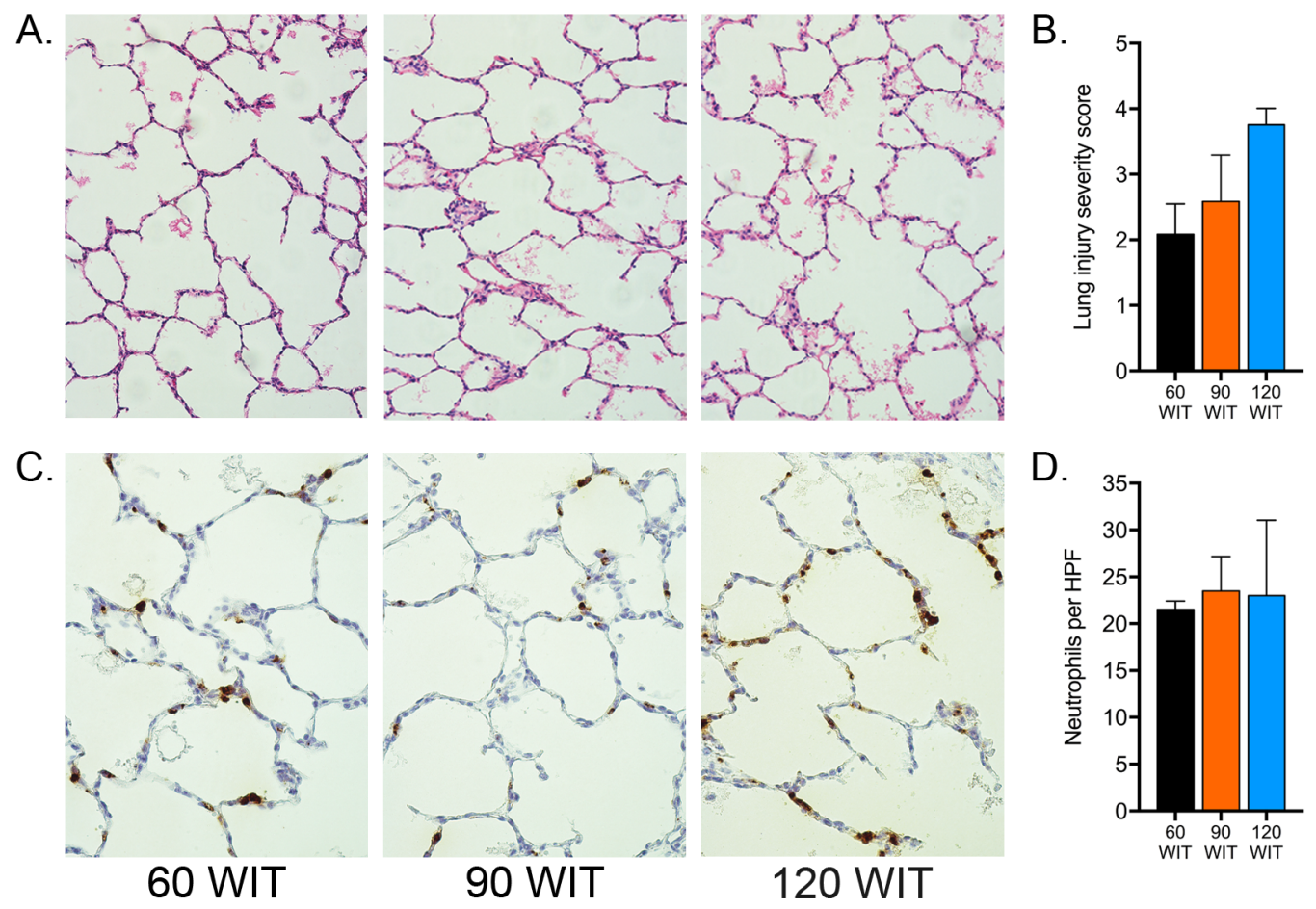

Figure 15: Lung histology and neutrophil counts at the end of ex vivo lung perfusion (EVLP). (A) Representative H\&E sections (20X magnification). (B) Lung injury severity scores. (C) Representative sections immunostained for neutrophils (40X magnification, neutrophils are stained brown). (D) Neutrophil counts per high-powered field (HPF) as quantified from immunostained sections. $60 \mathrm{WIT}$, lungs procured after hypoxic cardiac arrest and $60 \mathrm{~min}$ of warm ischemia; 90 WIT, lungs procured after hypoxic cardiac arrest and 90 min of warm ischemia; 120 WIT, lungs procured after hypoxic cardiac arrest and 120 min of warm ischemia.

\section{Discussion}

The present study sought to evaluate the effect of warm ischemia after circulatory arrest on ex vivo lung function, using a porcine DCD model. After confirming asystole, donor lungs were exposed to 60,90 , or 120 minutes of warm ischemia prior to 4 hours of EVLP. Transplant suitability was determined at the completion of EVLP using the Toronto exclusion criteria $\left(\mathrm{PO}_{2} / \mathrm{FiO}_{2}<400 \mathrm{mmHg}\right.$, greater than $15 \%$ increase in PA pressure, and greater than $15 \%$ deterioration of airway pressures and compliance). [39, 59] All three groups (60 WIT, 90 WIT, and 120 WIT) met the criteria for oxygenation, with no significant difference between groups in 
final $\mathrm{PO}_{2} / \mathrm{FiO}_{2}$. PA pressure increases were acceptable for transplantation in groups 60 WIT and 120 WIT, but not in group 90 WIT $(30.8 \pm 5.0 \%)$. All three groups met the transplant criteria for acceptable changes in airway pressures and compliance. There were also no significant differences between groups in edema accumulation, histologic lung injury severity scores, or neutrophil counts. These results suggest that DCD lungs with WIT up to 120 min still meet transplant criteria after 4 hours of EVLP.

In recent years, many institutions both in the U.S. and internationally have expanded their procurement protocols to accept Maastricht category III "controlled" DCD lungs. [9, 61] These patients undergo planned withdrawal of treatment, often in the operating room, with transplant teams nearby, who begin organ procurement shortly after confirmation of death. [11] "Uncontrolled" Maastricht category I and II donors however have yet to be incorporated into routine transplant protocols due to the associated logistic, physiologic, and ethical challenges, including timely notification of the procurement team, sufficient communication with family members, and proper management of the donor. [62] One center in Spain was been able to navigate the challenges associated with patients who die outside of the hospital and have reported their experience with 5 successful transplants. $[63,64]$

For the present study, we sought to better understand some of the associated physiologic challenges following hypoxic cardiac arrest using our established porcine model of lung procurement. Although this model does not reflect all the various pathways that can lead to circulatory arrest, it does parallel donors who suffer respiratory arrest, which may include prolonged hypoxia from long-standing pulmonary disease and acute respiratory emergencies from airway obstructions and drowning. Published data in a non-EVLP porcine lung transplant 
model show that pre-mortem hypoxia is associated with significant deterioration in graft function. [65]

The present study monitored all 15 animals with frequent ABGs and continuous cardiac monitoring during the hypoxic period in order to better characterize the physiologic changes associated with this model. Certain parameters $\left(\mathrm{pH}, \mathrm{PaCO}_{2}, \mathrm{HCO}_{3}\right.$, and base excess) were abnormal with a few minutes while others remained within the normal range for longer (MAP, lactic acid levels, $\mathrm{PO}_{2}$, and heart rate). We believe this data is valuable in helping to understand the quality of lungs that can be expected after certain types of death. Organs exposed to the same amount of warm ischemia following a $22 \mathrm{~min}$ period of hypoxia with severe physiologic derangements will likely function differently compared to lungs procured after sudden cardiac arrest.

After exposure to increasing amounts of warm ischemia, lungs from all three groups met all transplant criteria after 4 hours of EVLP, except the 90 WIT group on one parameter (PA pressure increase greater than 15\%). Additionally, when oxygenation capacity, airway pressures, and compliance were compared between groups, there were no significant differences after 4 hours of EVLP. This finding highlights the value of an ex vivo assessment of lungs prior to determining transplant suitability as it appears that increasing WIT alone (up to $120 \mathrm{~min}$ ) does not predict lung function after EVLP. For use of uncontrolled DCD lungs to become a reality, additional studies will be necessary to determine a defined upper limit of acceptable WIT. It likely will be prudent to procure all available donor lungs within a large window of warm ischemic exposure and assess them on EVLP prior to determining transplant suitability.

In the present study, EVLP provided a useful platform for assessing graft quality, but did not confer much in terms of reconditioning. EVLP significantly improved oxygenation capacity 
for groups 60 WIT and 90 WIT between hours 1 and 4, but not for group 120 WIT. PA pressures were significantly worse at the completion of EVLP for group 90 WIT, but not different for either 60 WIT or 120 WIT. It is not apparent why PA pressure increased so consistently for group 90 WIT and not for the other two groups. Airway pressures and compliance were not significantly different between hours 1 and 4 of EVLP for all three groups, however group 120 WIT appeared to benefit the most, with static compliance improvements of $25.2 \pm 23.2 \%$ and dynamic compliance improvements of $15.6 \pm 16.3 \%$. Despite the lack of a statistical difference in improvement between hours 1 and 4 of EVLP for most parameters, all parameters at the completion of EVLP except for PA pressures in group 90 WIT were within acceptable limits for transplantation. Additionally, supporting data such as pulmonary edema accumulation, histologic lung injury severity scores, and neutrophil counts were not different between groups. Although the definitive test will be lung function after transplantation, it appears as though increasing warm ischemia alone does not worsen lung function at the completion of 4 hours of ex vivo assessment.

Although Maastricht category I and II lungs exist as a potential solution to the supplydemand mismatch, there are significant hurdles that must be overcome before they can become an acceptable, routine option. [66] Heparin was not administered to animals in the present study, as any pre-mortem interventions will likely not be possible. Clot within the pulmonary vasculature was present in most donor lungs procured after 90 and 120 min of warm ischemia, but was able to be flushed out with Perfadex. Published data on non-heparinzed category III lungs is encouraging but warm ischemia times are shorter under those circumstances. [67, 68] Other uncertainties include the cause of death (i.e. respiratory arrest, cardiac arrest, exsanguination) and any resuscitation efforts that are attempted (rescue breathing, chest 
compressions). This variability in patient presentation makes EVLP a near necessity in order to fully assess graft function and potentially rehabilitate damaged organs. Our laboratory and others have evaluated various treatment options delivered during EVLP that may help recondition injured lungs. [56, 69-71] As these therapies are developed, the likelihood increases that we may one day have protocols that allow for routine, successful transplantation of Maastricht category I and II lungs.

The present study does have limitations. First, although this study was designed to set a baseline for acceptable warm ischemic injury by testing ex vivo lung function, the findings are limited because the organs were not subsequently transplanted into recipient animals and allowed to reperfuse. The effect of ischemia-reperfusion injury after transplant on graft function will be an important next step to investigate. Second, there is inherent variability present in large animal translational studies. Our study was limited to $\mathrm{n}=3$ for the 60 WIT group and $\mathrm{n}=6$ for the 90 and 120 WIT groups, and it is possible that the use of higher numbers of animals per group could have revealed small but significant differences between groups in some parameters (i.e. lung injury scores, Figure 15B). Based on previous work from our laboratory using 60 min of warm ischemia after hypoxic cardiac arrest, we limited the number of animals randomized to group 60 WIT to three. [38] Third, our study was also limited by the hypoxia method used to induce cardiac arrest. While this may not reflect all methods by which potential human uncontrolled DCD donors die, it provided consistent, reproducible injury for this experiment. Finally, our findings are limited by not having a group that failed to meet transplant criteria after EVLP, which would have helped define the upper limit of acceptable WIT.

In conclusion, for uncontrolled DCD donors to become a realistic source of lungs for transplantation, understanding the effect of increasing WIT after different types of death is 
necessary. The present study shows that DCD lungs exposed to increasing WIT up to $120 \mathrm{~min}$ after hypoxic cardiac arrest still meet transplant criteria after 4 hours of normothermic EVLP. As the field of lung transplantation continues to evolve to meet the ever-growing demand for organs, DCD lungs exposed to warm ischemia up to 120 min may eventually become acceptable for transplantation in conjunction with routine use of EVLP. 


\section{Noninvasive Diagnosis of Lung IR Injury by SPECT Imaging}

The diagnostic utility of in vivo leukocyte labeling with ${ }^{99 \mathrm{~m}}$ Tc-cFLFLF, an FPR1 peptide ligand, is investigated in a murine model of left lung IRI. SPECT imaging is utilized as a noninvasive method to diagnose and monitor resolution of lung IR. Fluorescence imaging with Cy7-cFLFLF is explored as an alternative strategy. 


\section{PROJECT \#3}

Use of cFLFLF, a Formyl Peptide Receptor Ligand, and SPECT Imaging to Noninvasively Diagnose and Monitor Lung Ischemia-Reperfusion Injury

Eric J. Charles MD ${ }^{1}$, Mahendra D. Chordia MBBS ${ }^{2}$, Yunge Zhao MD PhD ${ }^{1}$, J. Hunter Mehaffey MD MSCI ${ }^{1}$, David K. Glover $\mathrm{PhD}^{3}$, Irving L. Kron $\mathrm{MD}^{1}$, Dongfeng Pan $\mathrm{PhD}^{2}$, Ashish K. Sharma MBBS $\mathrm{PhD}^{1}$, Victor E. Laubach $\mathrm{PhD}^{1}$

Departments of ${ }^{1}$ Surgery, ${ }^{2}$ Radiology and Medical Imaging, and ${ }^{3}$ Medicine, University of Virginia, Charlottesville, Virginia

\section{Status of Research: MANUSCRIPT IN PREPARATION}




\begin{abstract}
Background: Primary graft dysfunction (PGD) after lung transplantation is a major cause of morbidity and mortality. Currently, no specific methods exist to diagnose ischemia-reperfusion injury (IRI), the major precursor to PGD. We hypothesized that use of ${ }^{99 m}$ Tc-cFLFLF, a formyl peptide receptor 1 ligand conjugated with technetium-99m, would permit in vivo leukocyte labeling and noninvasive imaging of lung IRI using single-photon emission computed tomography (SPECT).
\end{abstract}

Methods: A murine model of IRI via left lung hilar occlusion was used. Following 1 hour of ischemia, SPECT was performed after 2, 12, or 24 hours of reperfusion, with injection of ${ }^{99 \mathrm{~m}} \mathrm{Tc}-$ cFLFLF 2 hours prior to imaging. Lung maximum intensity projections (MIP) and biodistribution of ${ }^{99 m}$ Tc-cFLFLF were correlated with lung function.

Results: Left-to-right lung ratios of MIP and probe uptake were 3.8- and 2.8-fold higher, respectively, compared with sham animals, which correlated with resolution of injury over time as measured by lung function and histologic presence of neutrophils. Findings were confirmed using Cy7-cFLFLF and fluorescence imaging.

Conclusions: Results suggest that SPECT imaging using ${ }^{99 \mathrm{~m}} \mathrm{Tc}-\mathrm{cFLFLF}$ enables quantifiable, noninvasive diagnosis of lung IRI and permits monitoring of injury resolution over time. Clinical application of ${ }^{99 \mathrm{~m}}$ Tc-cFLFLF as functional imaging probe may permit early, accurate diagnosis of PGD, enabling targeted interventions and improved outcomes after lung transplantation. 


\section{Introduction}

Lung transplantation has advanced dramatically since its inception in 1963, but still lags behind other solid organs in terms of donor organ utilization and transplant success rates.[14, 72] As the supply of acceptable donor lungs remains low, wait list mortality rates continue to rise.[3] One reason that most institutions have strict acceptance criteria for donor lungs is the relatively high rate of primary graft dysfunction (PGD).[73] PGD is a clinical diagnosis that describes acute lung injury that occurs within the early postoperative period after lung transplantation and is characterized by reduced oxygenation capacity and progressive lung failure.[13] PGD occurs in up to $30 \%$ of patients and is the leading cause of early morbidity and mortality.[13-15] Rates of late organ failure (bronchiolitis obliterans and chronic rejection) are also significantly higher in patients diagnosed with PGD.[16]

The underlying pathophysiology responsible for PGD is ischemia-reperfusion injury (IRI).[19] Lung IRI occurs rapidly after transplant and involves disruption of endothelial and epithelial cells as well as robust activation of innate immune cells such as neutrophils, alveolar macrophages, and natural killer T-cells.[20-25] Reactive oxygen species and proinflammatory cytokines (IL-8, IL-12, IL-17, IL-18, TNF- $\alpha$ ) are generated leading to activation of multiple inflammatory pathways involving NADPH oxidase and NF-kB.[22, 26-32] Expression of endothelial cell adhesion molecules is up-regulated, leading to leukocyte attraction and migration into the interstitium and perpetuation of further tissue damage.[33] Clinically, lung IRI is characterized by increased vascular permeability and resistance, accumulation of pulmonary edema, poor gas exchange, reduced oxygenation capacity, and increased pulmonary artery (PA) pressure. [26] 
The International Society for Heart and Lung Transplantation grading system for severity of PGD uses the allograft's oxygenation capacity (ratio of partial pressure of oxygen in arterial blood to the fraction of inspired oxygen $\left.\left[\mathrm{PaO}_{2} / \mathrm{FiO}_{2}\right]\right)$ and the presence of infiltrates of chest radiograph, both of which are general markers of lung dysfunction.[14] There are no clinically available methods for in vivo leukocyte labeling that can identify cell-type specific inflammation occurring in the recently transplanted lung.[18] This inability to diagnose IRI, and thus PGD, early may contribute to high rates of morbidity and mortality. Strategies are needed that allow for early and accurate diagnosis of IRI (prior to onset of PGD) to allow for earlier initiation of targeted treatment strategies as well as to monitor response to therapy and resolution of injury over time.

Our laboratory has published previously on the development of a novel formyl peptide receptor 1 (FPR1) ligand that can be used as an in vivo leukocyte-specific imaging probe when conjugated with a radioisotope or near-infrared fluorescent dye.[74] Cinnamoyl-F-(D)L-F-(D)LF-K (cFLFLF) is a synthetic, unnatural peptide with high affinity for FPR1 that does not induce neutrophil activation and when linked with polyethylene glycol (PEG) has a favorable pharmacokinetic profile. $[74,75]$ FPRs are transmembrane G-protein coupled receptors that are highly expressed on polymorphonuclear leukocytes (PMNs) and monocytes when exposed to pro-inflammatory stimuli and mediate cell trafficking to areas of tissue damage or bacterial presence.[76] When complexed with technetium-99m, cFLFLFK-PEG-HYNIC- ${ }^{99 \mathrm{~m}} \mathrm{Tc}\left({ }^{99 \mathrm{~m}} \mathrm{Tc}-\right.$ cFLFLF) can be visualized using single-photon emission computed tomography (SPECT), allowing for noninvasive visualization of PMN activity at sites of tissue injury or inflammation.[75] The objective of this study was to investigate the diagnostic utility of in vivo leukocyte labeling with ${ }^{99 \mathrm{~m}}$ Tc-cFLFLF in a murine model of left lung IRI. We hypothesized that 
use of ${ }^{99 \mathrm{~m}}$ Tc-cFLFLF with SPECT would permit noninvasive and quantitative imaging of leukocyte activity at sites of tissue injury and allow for monitoring of IRI resolution over time.

\section{Materials and Methods}

The current study conformed to the standards set by the National Institutes of Health in the 2011 Guide for the Care and Use of Laboratory Animals, $8^{\text {th }}$ edition. The study protocol was reviewed and approved by the Animal Care and Use Committee at the University of Virginia.

Imaging Probe: ${ }^{99 m}$ Tc-cFLFLF

Our laboratory has published previously on the synthesis and validation of cFLFLF, a peptide ligand antagonist to FPR1 on PMNs.[74, 75] We have reported the use of cFLFLFKPEG- ${ }^{64} \mathrm{Cu}$ as a novel neutrophil-specific positron emission tomography (PET) imaging agent, as well as the use of cFLFLF-PEG-TKPPR $-{ }^{99 \mathrm{~m}} \mathrm{Tc}$ as a neutrophil targeting heterobivalent SPECT imaging agent.[74, 75] We have also reported on the synthesis of a Cy7-labeled version of cFLFLF (Cy7-cFLFLF) for noninvasive near infrared fluorescence imaging.[77] In the present study, we use PEGylated cFLFLF conjugated with hydrazinonicotinamide (HYNIC) and labeled with ${ }^{99 \mathrm{~m}}$ Tc (cFLFLFK-PEG-HYNIC- ${ }^{99 \mathrm{~m}}$ Tc, abbreviated ${ }^{99 \mathrm{~m}}$ Tc-cFLFLF) for SPECT imaging experiments. We also utilize cFLFLFK-PEG coupled with commercially available Cy5 or Cy7NHS ester (Sigma Aldrich, St. Louis, MO) (abbreviated Cy5-cFLFLF or Cy7-cFLFLF) for confocal microscopy and fluorescence imaging experiments. 


\section{Lung IRI model}

Wild-type mice (C57BL/6, 8-12 weeks old; The Jackson Laboratory, Bar Harbor, ME) were used for all in vivo experiments. An established, reproducible murine model of left lung IRI via temporary hilar occlusion was used as illustrated in Figure 1. [22, 24] Mice were anesthetized with $2 \%$ inhaled isoflurane, intubated, and ventilated with room air at 120 breaths/minute. Heparin (20 U/kg; Hospira Inc., Lake Forest, IL) was administered via the right external jugular vein. A left anterolateral thoracotomy was made at the third intercostal space to expose the hilum of the left lung. Using a small olive tip J-hook cannula, a 6-0 Prolene suture (Ethicon Inc., Somerville, NJ) was passed around the left hilum. A Rummel tourniquet was fashioned with PE60 polyethylene tubing and a surgical clip and was used to temporarily occlude the hilar structures (left PA, left bronchus, left pulmonary veins). The incision was closed with surgical clips, analgesia was administered (intraperitoneal buprenorphine, $0.2 \mathrm{mg} / \mathrm{kg}$ ), and the animal was weaned from anesthesia and extubated when breathing spontaneously (Figure 16A). Animals were returned to their cage for the prescribed 1-hour period of left lung ischemia. Animals were then re-anesthetized with $2 \%$ isoflurane and the Rummel tourniquet was removed from the hilum of the left lung to permit reperfusion (Figure 16B). Animals were returned to their cages and underwent 2, 12, or 24 hours of reperfusion. Sham animals underwent all steps described except for application of the Rummel tourniquet to occlude the hilum of the left lung. Two hours prior to completion of the reperfusion period, animals received a tail-vein injection of the cFLFLF imaging probe (labeled with ${ }^{99 \mathrm{~m}} \mathrm{Tc}$ or $\mathrm{Cy} 7$, Figure 16C). At the conclusion of the reperfusion period, animals underwent functional evaluation followed by SPECT/CT or fluorescence imaging (Figure 16D). 


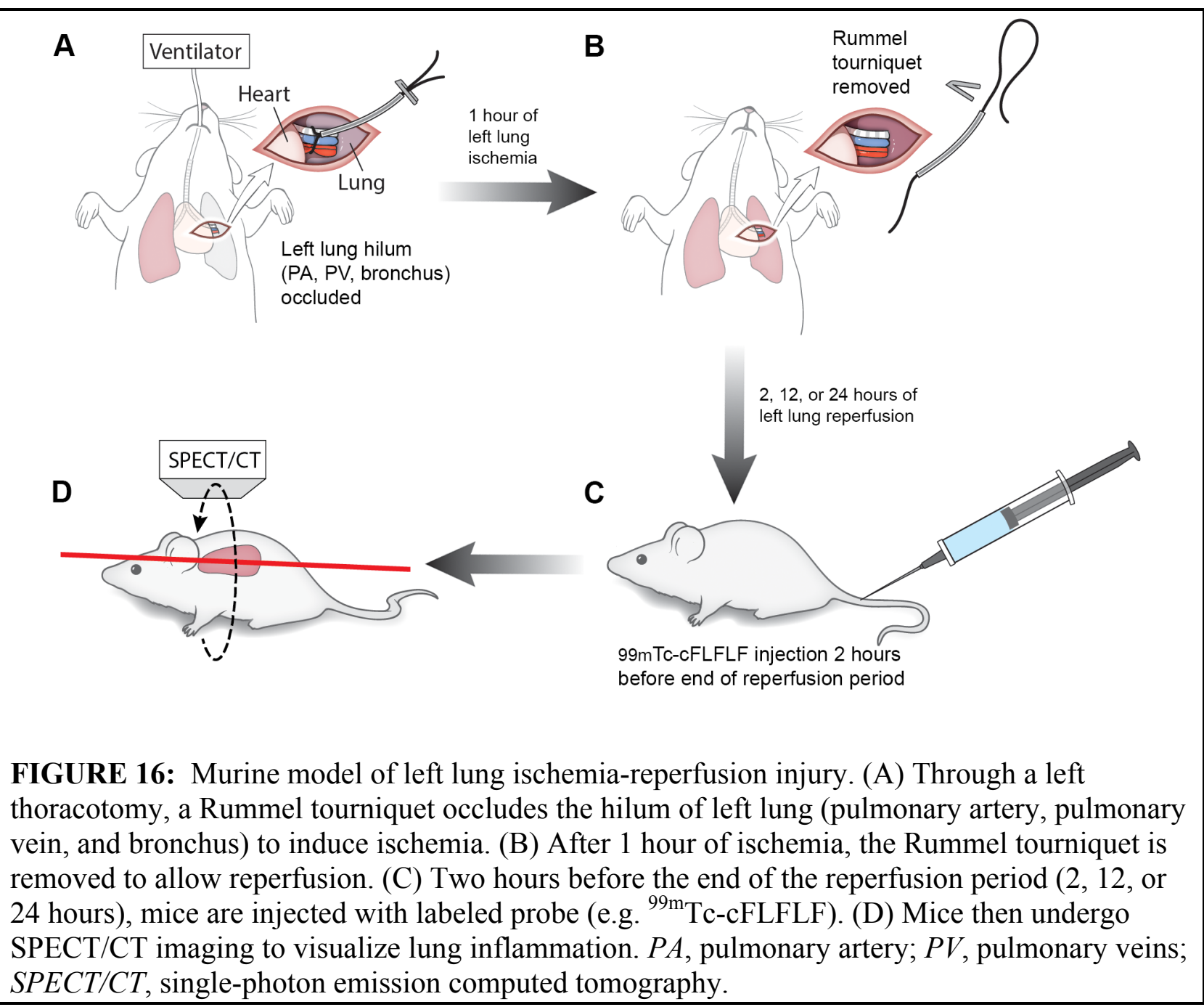

\section{Pulmonary Function}

After the reperfusion period, pulmonary function (compliance, airway resistance, and PA pressure) was measured in sham and IR animals ( $\mathrm{n}=5-10 /$ group) using an isolated, bufferperfused lung apparatus (Hugo Sachs Elektronik, March-Huggstetten, Germany) as previously described. [78] Briefly, mice were anesthetized with a mixture of ketamine/xylazine, intubated via tracheotomy and ventilated (tidal volume: $7 \mu \mathrm{l} / \mathrm{g}$ body weight, rate: 100 ventilations/minute, positive end-expiratory pressure: $2 \mathrm{cmH}_{2} \mathrm{O}$ ), and then exsanguinated via transection of the abdominal aorta and inferior vena cava. The main PA via the right ventricle was cannulated to 
allow for perfusion of the lungs with Krebs-Henseleit buffer (flow rate: $60 \mu \mathrm{l} / \mathrm{g}$ body

weight/minute) and a left ventriculotomy was made to drain perfusate. After a 5-minute period of equilibration, lung function data was captured over an acquisition period of 5 minutes using PULMODYN data acquisition software (Hugo Sachs Elektronik).

\section{SPECT/CT Imaging}

Separate groups of sham and IR animals ( $\mathrm{n}=6 /$ group) were used for SPECT imaging with

${ }^{99 \mathrm{~m}}$ Tc-cFLFLF. Two hours prior to the end of the reperfusion period, animals received a $200 \mu \mathrm{l}$ tail vein injection of ${ }^{99 \mathrm{~m}}$ Tc-cFLFLF (40-60 MBq; Figure 16C). After 2 hours, animals were anesthetized with $1 \%$ isoflurane and $360^{\circ} \mathrm{SPECT} / \mathrm{CT}$ imaging was performed (Figure 16D). A microSPECT/CT scanner designed and built at the University of Virginia was used.[75, 79] Briefly, the anesthetized mouse was positioned supine on a 1-inch diameter carbon fiber halfcylinder tube located at the axis of rotation of the scanner gantry. Anesthesia was maintained with continuous inflow of isoflurane via a nose cone. Sequential CT and SPECT imaging scans were acquired by transitioning the animal from one subsystem to the other along the axis of rotation, allowing for consistent fusion of CT and SPECT images using stored offset parameters. A custom-written interactive data language program was used with the $\mathrm{CT}$ images for detector sensitivity uniformity correction and dark count subtraction. The images were reconstructed using a Feldkamp three-dimensional filtered back projection algorithm (COBRA, Exxim, Inc., Pleasanton, CA). A custom-written interface using Kmax software (Sparrow Corp., Port Orange, FL) was used to acquire SPECT images with a gamma camera set to $10 \mathrm{~cm} \times 10 \mathrm{~cm}$ view. A custom-written maximum-likelihood expectation-maximization algorithm was used to reconstruct SPECT images.[75] Maximum intensity projections (MIP) were obtained from ten 
random slices through each lung and used to calculate an average MIP value per lung. Values are reported as the ratio of left lung MIP to right lung MIP.

At the completion of SPECT/CT imaging, animals were euthanized in a $\mathrm{CO}_{2}$ chamber. All major organs (lungs, heart, kidneys, liver, and spleen) were harvested, weighed, and placed in a gamma-well counter (PerkinElmer Inc., Waltham, MA). Relative radioactivity of each organ was normalized to injected dose, tissue weight, and animal body weight. Organ biodistribution of ${ }^{99 \mathrm{~m}}$ Tc-cFLFLF is expressed as a percentage of total observed radioactivity. Lung ${ }^{99 \mathrm{~m}} \mathrm{Tc}$ uptake is reported as the ratio of left lung to right lung relative radioactivity.

Near-infrared Fluorescence Imaging with Cy7-cFLFLF

Separate groups of sham and IR (2 hours reperfusion) animals were injected with Cy7cFLFLF and underwent fluorescence imaging. All animals received a $100 \mu$ l tail vein injection of 2nM Cy7-cFLFLF upon reperfusion. After 2 hours, animals were euthanized in a $\mathrm{CO}_{2}$ chamber and both lungs were harvested. Due to the limited penetration and scattering of fluorescence signal in black fur mice, lungs were imaged ex vivo using an IVIS Spectrum Imaging System (excitation 745 nm, emission 775 nm; PerkinElmer, Inc., Waltham, MA). The images were processed and analyzed with Living Imaging software, version 4.0 (PerkinElmer, Inc.). Total fluorescence radiance was obtained for each lung by designating a region of interest that incorporated the entire lung field. One additional animal per group was shaved on the back and the animal was imaged under anesthesia (1\% isoflurane via nose cone) using the IVIS Spectrum Imaging System. 
Neutrophil Immunohistochemistry

Left lungs from separate groups of sham and IR animals were harvested and fixed with $10 \%$ buffered formalin for 24 hours and then placed in 70\% ethanol, paraffin-embedded and sectioned. Vectastain ABC kit (Vector Laboratories, Burlingame, CA) was used to immunostain neutrophils as previously described. $[41,78]$ Primary antibody used was rat anti-mouse neutrophil antibody (AbD Serotec, Raleigh, NC) and secondary antibody used was alkaline phosphatase-conjugated anti-rat IgG (Sigma Aldrich). Fast-Red (Sigma Aldrich) was used to detect neutrophil signal. Purified normal rat IgG (eBioscience, San Diego, CA) was used as a negative control. Five semistandardized fields per lung were counted in a blinded fashion at 20X magnification and averaged to obtain a final neutrophil count per high-powered field per left lung.

In vitro Neutrophil Incubation with Cy5-cFLFLF

Spleens were harvested from wild-type mice (C57BL/6, 8-12 weeks old; The Jackson Laboratory, Bar Harbor, ME) and dissociated into a single-cell suspension using a gentleMACS ${ }^{\mathrm{TM}}$ Dissociator (Miltenyi Biotec, Auburn, CA). Neutrophils were isolated via positive selection using a murine Neutrophil Isolation Kit (Miltenyi Biotec, Auburn, CA) with MACS Columns and Separator (Miltenyi Biotec, Auburn, CA). Neutrophils were then plated $\left(1 \times 10^{5}\right.$ cells per well) onto chamber slides (Thermo Fisher Scientific, Waltham, MA). Phorbol 12-myristate 13-acetate (PMA, Sigma-Aldrich) was added to select wells (final concentration of $32 \mathrm{nM}$ ) to stimulate neutrophil activation. Slides were then incubated for 2 hours at $37^{\circ} \mathrm{C}$ with $5 \% \mathrm{CO}_{2}$. All cells were then fixed using a 50:50 mixture of methanol and acetone and washed 
three times with PBS. Cy5-cFLFLF (1nM), anti-Ly6G-FITC (5 $\mu \mathrm{g} / \mathrm{mL}$; Biolegend, San Diego, CA), and DAPI ( $1 \mu \mathrm{g} / \mathrm{mL}$; Invitrogen, Carlsbad, CA) were added to all wells, along with blocking buffer to reduce non-specific binding (5\% Blotto in PBS, $0.5 \mathrm{~mL} /$ well, Santa Cruz Biotechnology, Dallas, TX). Confocal microscopy was performed and images were captured at $200 X$.

Immunofluorescence Staining with Cy7-cFLFLF

Whole-lung tissue specimens after sham and IR 2-hr were immediately fixed in $4 \%$ freshmade paraformaldehyde (PFA) for 24 hours. The fixed samples were embedded in paraffin and sectioned into $5 \mu \mathrm{m}$ slices. The double immunofluorescence staining was performed as described in our previous methods $[80,81]$. Briefly, paraffin was removed from the sections with xylene, then washed and rehydrated with $100 \%, 95 \%$ and $70 \%$ ethanol. The tissue sections were incubated with anti-mouse Ly6G-FITC antibody (1:100), and Cy7-cFLFLF peptide (2nM, 1:100) at $4^{\circ} \mathrm{C}$ overnight. The sections were then sealed with ProLong Gold Antifade Mountant with DAPI (Invitrogen, Carlsbad, CA). Images were obtained with an Olympus IX81 inverted confocal microscope (Olympus Corp., Tokyo, Japan) with a CCD camera at 200X and 400X magnification.

\section{Statistical Analysis}

Groups were compared using two-tailed Student's t test or one-way analysis of variance (ANOVA) with a post-hoc Bonferroni correction for multiple comparisons. $\mathrm{P}<0.05$ was used to determine statistical significance. All values are reported as mean \pm standard error of the mean. Prism 7 (GraphPad Software, La Jolla, CA) was used for all statistical analyses. 


\section{Results}

Lung Function after IRI

Pulmonary function measurements (compliance, airway resistance, and PA pressure)

after IR demonstrated peak injury after 2 hours of reperfusion with resolution of injury over time.

Pulmonary compliance was significantly reduced after IR compared with sham (IR 2-hr: 3.3 \pm 0.1

vs. Sham: $6.5 \pm 0.4 \mu \mathrm{L} / \mathrm{cmH}_{2} \mathrm{O}, \mathrm{p}<0.0001$ ) and improved with increasing reperfusion time (IR 12hr: $3.9 \pm 0.2 \mu \mathrm{l} / \mathrm{cmH}_{2} \mathrm{O}, \mathrm{p}>0.05$ vs. IR 2-hr; IR 24-hr: 5.0 $0.2 \mu \mathrm{l} / \mathrm{cmH}_{2} \mathrm{O}, \mathrm{p}=0.02$ vs. IR 12-hr,

Figure 17A). Airway resistance was significantly higher after IR compared with sham (IR 2-hr:

$1.9 \pm 0.1$ vs. Sham: $\left.1.1 \pm 0.04 \mathrm{cmH}_{2} \mathrm{O} / \mu \mathrm{L} / \mathrm{s}, \mathrm{p}<0.0001\right)$ and improved with increasing reperfusion time (IR 12-hr: $1.5 \pm 0.1 \mathrm{cmH}_{2} \mathrm{O} / \mu \mathrm{L} / \mathrm{s}, \mathrm{p}=0.0005$ vs. IR 2-hr; IR 24-hr: $1.3 \pm 0.1 \mathrm{cmH}_{2} \mathrm{O} / \mu \mathrm{L} / \mathrm{s}$, p $>0.05$ vs. Sham, Figure 17B). PA pressure was significantly higher after IR compared with sham (IR 2-hr: $11.8 \pm 0.4$ vs. Sham: $5.7 \pm 0.2 \mathrm{cmH}_{2} \mathrm{O}, \mathrm{p}<0.0001$ ) and improved with increasing reperfusion time (IR 12-hr: $8.9 \pm 0.7 \mathrm{cmH}_{2} \mathrm{O}, \mathrm{p}=0.0004$ vs. IR 2-hr; IR 24-hr: $6.1 \pm 0.4 \mathrm{cmH}_{2} \mathrm{O}$, p $>0.05$ vs. Sham, Figure 17C).
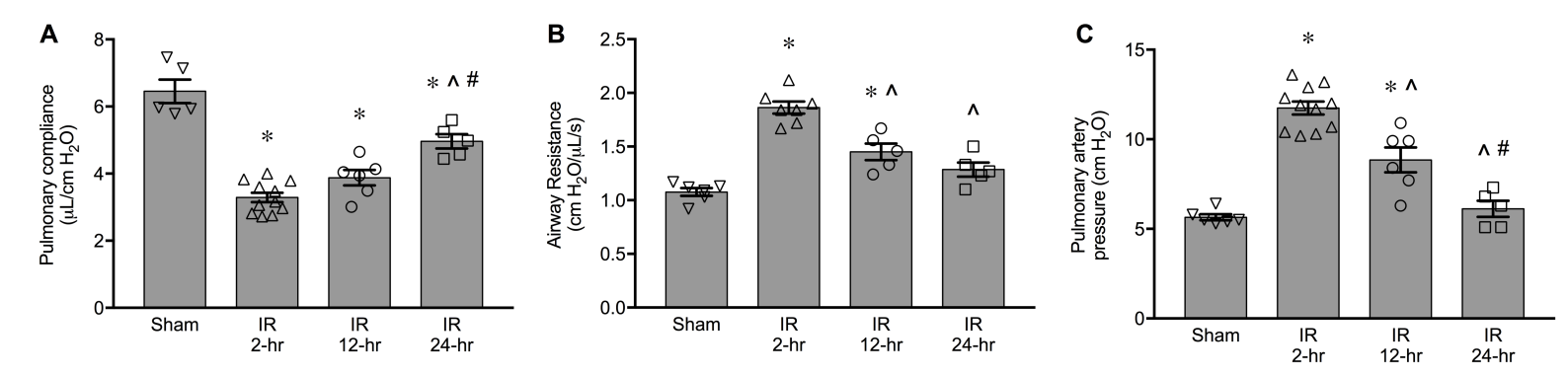

FIGURE 17: Lung function after ischemia-reperfusion (IR) demonstrating peak injury after 2 hours of reperfusion with resolution of injury over time. (A) Pulmonary compliance, (B) airway resistance, and (C) pulmonary artery pressure after 1 hour of left lung ischemia followed by 2 , 12, or 24 hours of reperfusion compared to sham animals. IR 2-hr, mice after 1 hour of left lung ischemia and 2 hours of reperfusion; IR 12-hr, mice after 1 hour of left lung ischemia and 12 hours of reperfusion; IR 24-hr, mice after 1 hour of left lung ischemia and 24 hours of reperfusion. ${ }^{*} \mathrm{p}<0.05$ vs. Sham, ${ }^{\wedge} \mathrm{p}<0.05$ vs. IR 2 -hr, $\# \mathrm{p}<0.05$ vs. IR $12-\mathrm{hr}$. 
SPECT/CT Imaging of Lung IRI with ${ }^{99 m}$ Tc-cFLFLF

In vivo leukocyte labeling with ${ }^{99 \mathrm{~m}} \mathrm{Tc}-\mathrm{cFLFLF}$ and SPECT imaging allowed for noninvasive diagnosis of left lung IRI after 2, 12, and 24 hours of reperfusion. Signal intensity demonstrated peak injury after 2 hours of reperfusion with reduction in signal intensity over time. Representative axial and coronal sections are shown in Figure 18A. Maximum intensity projections (MIP) in left lungs after 2 and 12 hours of reperfusion were significantly higher than sham mice (IR 2-hr: $4.2 \pm 0.3$ vs $1.1 \pm 0.04, \mathrm{p}<0.001$; IR 12 -hr: $2.1 \pm 0.2$ vs $1.0 \pm 0.04, \mathrm{p}<0.001$, Figure 18B). By 24 hours of reperfusion, there was no significant difference in MIP between IR and sham animals $\left(1.4 \pm 0.2\right.$ vs $1.2 \pm 0.04, \mathrm{p}=0.2$, Figure 18B). Total uptake of ${ }^{99 \mathrm{~m}} \mathrm{Tc}$-cFLFLF in left lungs was significantly higher after IRI at all three time-points compared with sham animals (IR 2-hr: $2.8 \pm 0.7$ vs $1.0 \pm 0.04, \mathrm{p}=0.02$; IR 12-hr: $1.8 \pm 0.1$ vs $1.0 \pm 0.1, \mathrm{p}<0.001$; IR 24-hr: $1.4 \pm 0.04$ vs $1.1 \pm 0.1, \mathrm{p}=0.003$, Figure 18C). Left-to-right lung ratios of MIP and probe uptake were 3.8and 2.8-fold higher, respectively, compared with sham animals, with both ${ }^{99 \mathrm{~m}}$ Tc-cFLFLF uptake and MIP diminishing with increasing reperfusion time, correlating with pulmonary function results and neutrophil counts. 

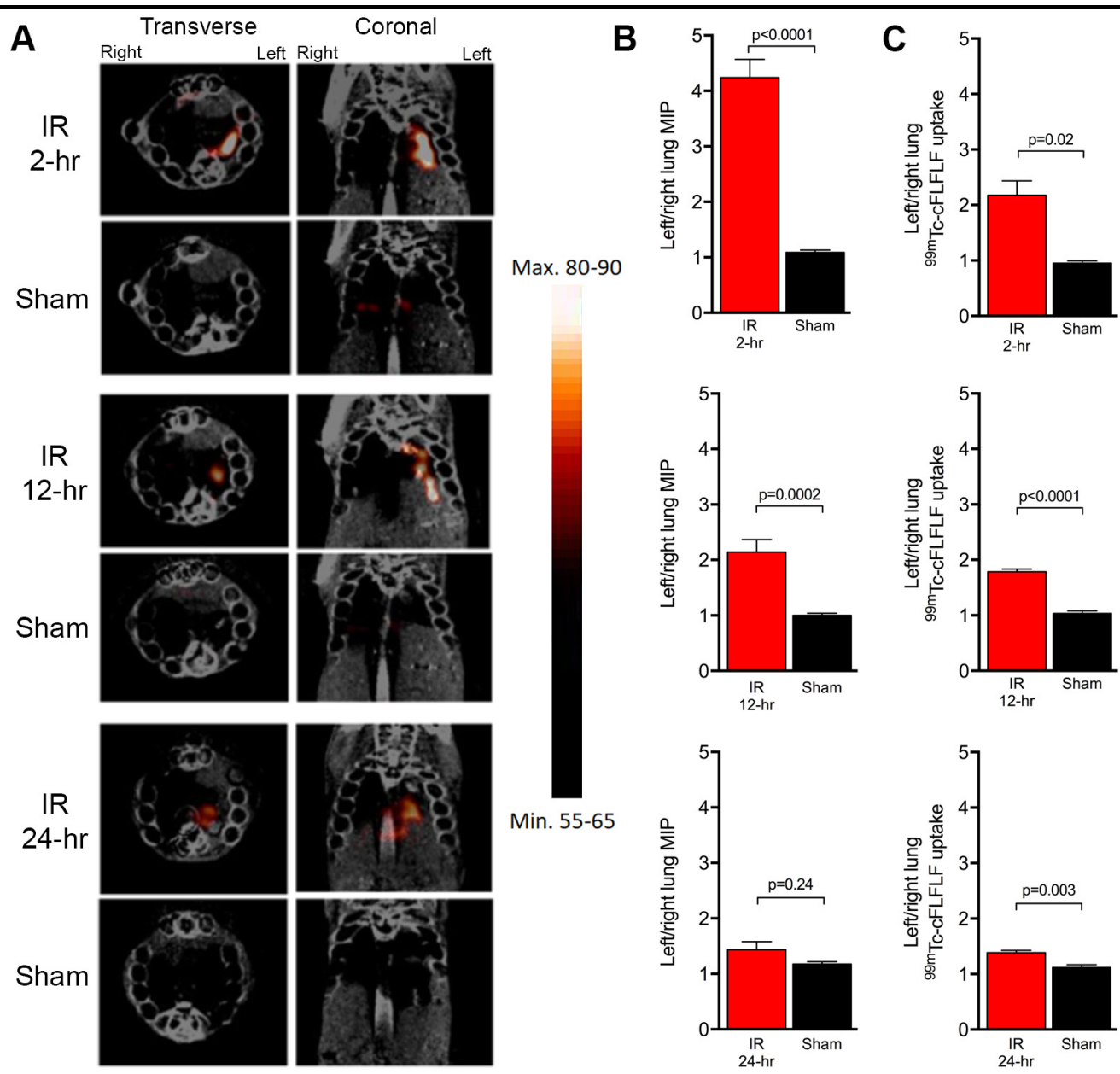

FIGURE 18: SPECT/CT imaging after lung ischemia-reperfusion (IR) demonstrating peak signal after 2 hours of reperfusion. (A) Representative SPECT/CT images after 1 hour of left lung ischemia followed by 2,12 , or 24 hours of reperfusion. Mice were injected with ${ }^{99 \mathrm{~m}} \mathrm{Tc}-$ cFLFLF two hours before the end of reperfusion. (B) Quantification of SPECT maximum intensity projections (MIP), reported as the ratio of left to right lung values. (C) Quantification of relative radioactivity in lungs measured ex vivo with a gamma-well counter, reported as the ratio of left to right lung ${ }^{99 \mathrm{~m}}$ Tc-cFLFLF uptake. $I R$ 2- $h r$, mice after 1 hour of left lung ischemia and 2 hours of reperfusion; IR 12-hr, mice after 1 hour of left lung ischemia and 12 hours of reperfusion; IR 24-hr, mice after 1 hour of left lung ischemia and 24 hours of reperfusion; MIP, maximum intensity projection.

\section{Organ Biodistribution of ${ }^{99 m}$ Tc-cFLFLF}

Organ biodistribution of ${ }^{99 \mathrm{~m}}$ Tc-cFLFLF was measured in mice undergoing 2 hours of reperfusion (Figure 19). Uptake was highest overall in kidneys representing excretion of excess probe. There was a significant difference in left lung uptake after IR compared with sham 
animals ( $12.4 \pm 1.3$ vs. $5.6 \pm 2.1$ relative $\%$ radioactivity, $\mathrm{p}=0.02)$ and a significant difference between left and right lung uptake in IR 2-hr animals (12.4 \pm 1.3 vs. $3.9 \pm 0.4$ relative $\%$ radioactivity, $\mathrm{p}<0.0001)$. Uptake was not significantly different between sham and IR animals in right lungs and in all other organs.

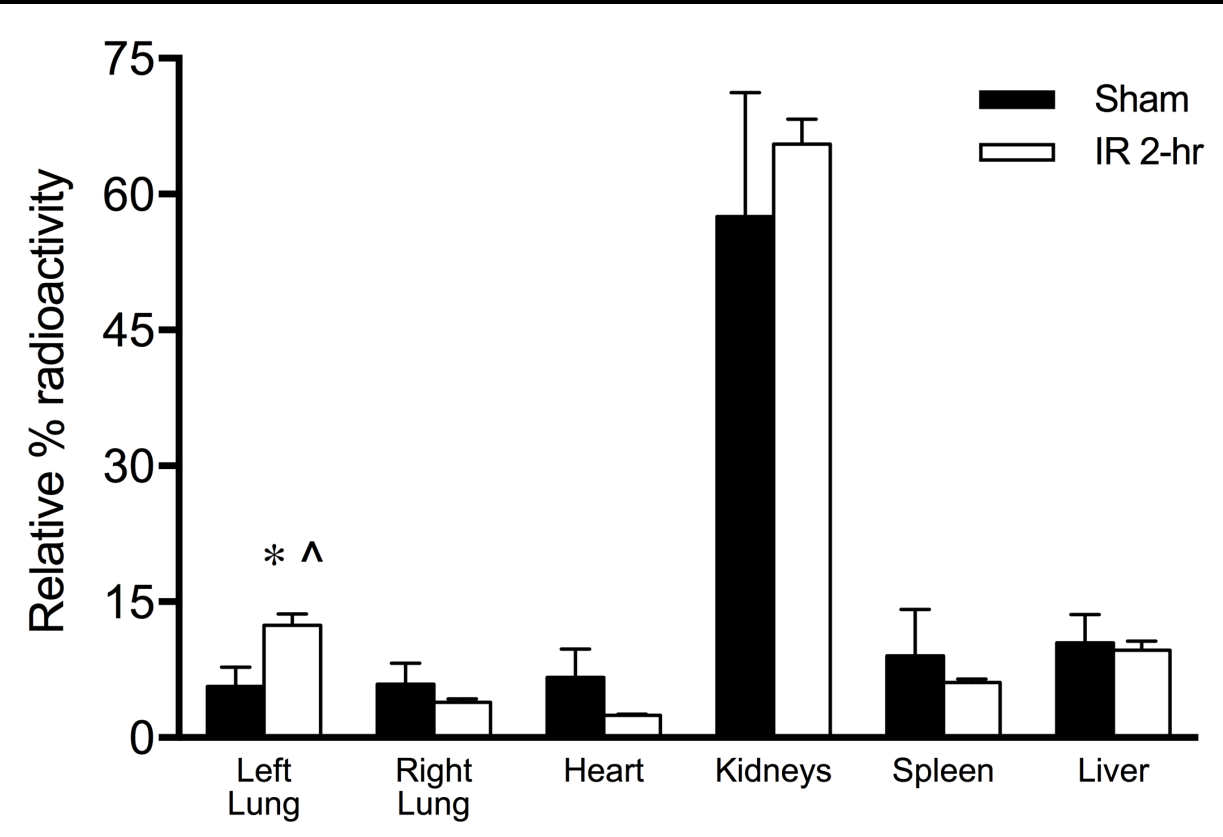

FIGURE 19: Organ biodistribution of ${ }^{99 \mathrm{~m}}$ Tc-cFLFLF uptake, reported as a percentage of total observed radioactivity in organs harvested and normalized to injected dose, tissue weight, and animal body weight demonstrating significant increase in left lung uptake in IR 2-hr animals. $I R$ 2 - $h r$, mice after 1 hour of left lung ischemia and 2 hours of reperfusion. * $\mathrm{p}=0.02 \mathrm{vs.} \mathrm{Sham} \mathrm{left}$ lung, ${ }^{\wedge} \mathrm{p}<0.0001$ vs. IR 2 -hr right lung.

Near-infrared Fluorescence Imaging of Lung IRI with Cy7-cFLFLF

SPECT imaging findings were confirmed using near-infrared Cy7-cFLFLF and fluorescence imaging. Active inflammation in left and right lungs after 2 hours of reperfusion was visualized ex vivo (Figure 20A). Signal quantification demonstrated total radiance efficiency that was significantly higher in IR 2-hour mice compared with sham animals (1.7 \pm 0.1 vs. $0.9 \pm 0.04$ left/right lung total radiance efficiency, $\mathrm{p}<0.0001$, Figure 20B). Although depth of 
fluorescence signal penetration is limited, in vivo imaging of left lung IRI in shaved, anesthetized mice was possible (Figure 20C).

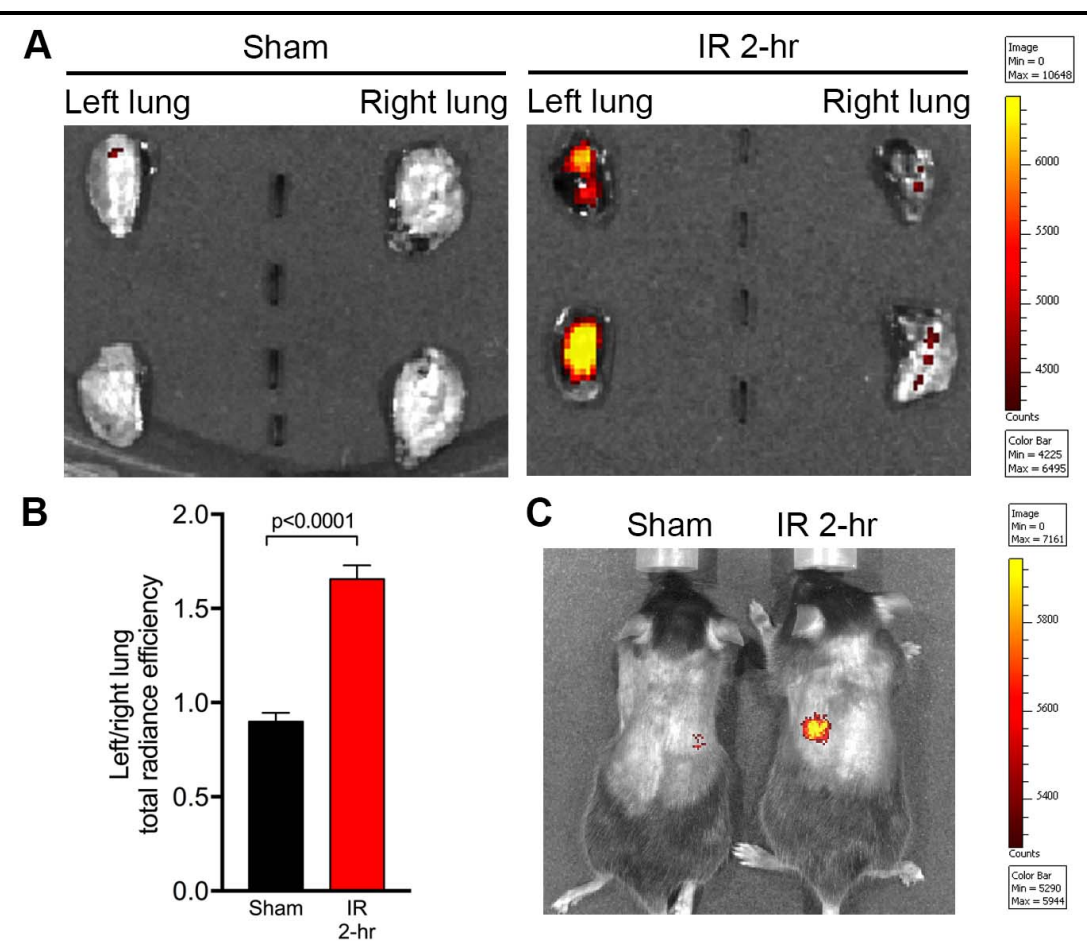

FIGURE 20: Fluorescence imaging of lung ischemia-reperfusion injury (IRI) demonstrating increased fluorescence signal in left lungs of IR 2-hr animals. Mice were injected with Cy7cFLFLF 2 hours prior to imaging. (A) Representative near-infrared (NIR) fluorescence images of ex vivo lungs from mice that underwent sham surgery or left lung IR with 2 hours of reperfusion. (B) Quantification of fluorescence intensities reported as ratio of left to right lung total radiance efficiency. (C) Representative fluorescence images of live, shaved, anesthetized mice demonstrating visualization of left lung IRI. IR 2-hr, mice after 1 hour of left lung ischemia and 2 hours of reperfusion.

Neutrophil Infiltration After IR

Immunostaining of left lung sections demonstrated significantly greater neutrophil infiltration after IR compared with sham animals ( $60 \pm 9$ neutrophils/HPF) at all three time points (IR 2-hr: $219 \pm 16$ neutrophils/HPF, p<0.0001; IR 12-hr: 179 23 neutrophils/HPF, p<0.0001; IR 24-hr: $154 \pm 9$ neutrophils/HPF, $\mathrm{p}<0.0001$, Figure 21). Neutrophil infiltration was highest after 2 hours of reperfusion and decreased over time. There was a significant difference between IR 2-hr and IR 24-hr IR animals ( $\mathrm{p}=0.01$, Figure 21). 


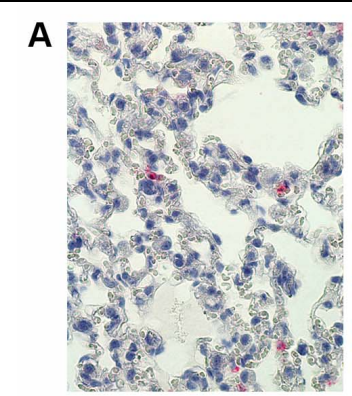

Sham

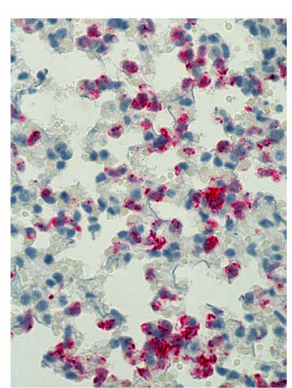

IR 2-hr

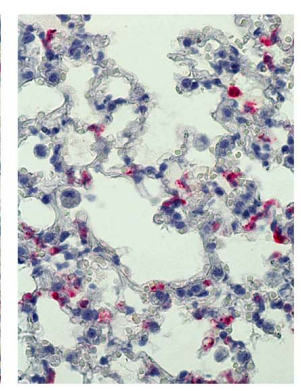

IR 12-hr

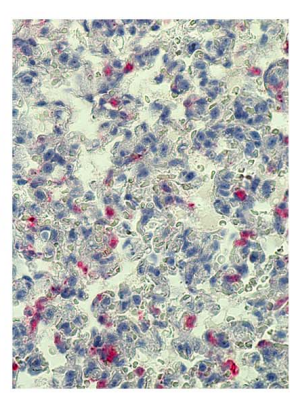

IR 24-hr

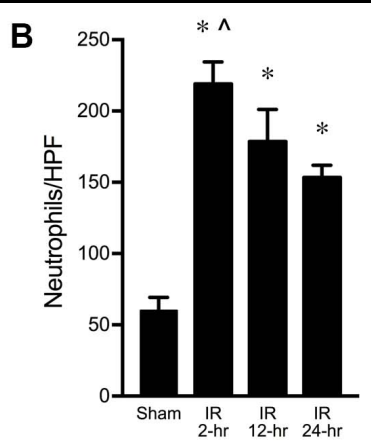

FIGURE 21: Neutrophil infiltration after ischemia-reperfusion (IR) demonstrating increased neutrophils in lung tissue sections from IR 2-hr animals. (A) Representative histology slides immunostained for neutrophils (red color, 40X magnification). (B) Quantification of neutrophils per high-powered field (HPF). IR 2-hr, mice after 1 hour of left lung ischemia and 2 hours of reperfusion; IR 12-hr, mice after 1 hour of left lung ischemia and 12 hours of reperfusion; IR 24$h r$, mice after 1 hour of left lung ischemia and 24 hours of reperfusion. ${ }^{*} \mathrm{p}<0.0001 \mathrm{vs}$. Sham, $\wedge$ $\mathrm{p}=0.01$ vs. $24-\mathrm{hr}$.

\section{Activated Neutrophils Bind cFLFLF}

Isolated murine neutrophils activated by PMA demonstrated significant Cy5-cFLFLF

binding that co-localized with DAPI and anti-Ly6G-FITC while unactivated neutrophils displayed minimal to no binding (Figure 22).

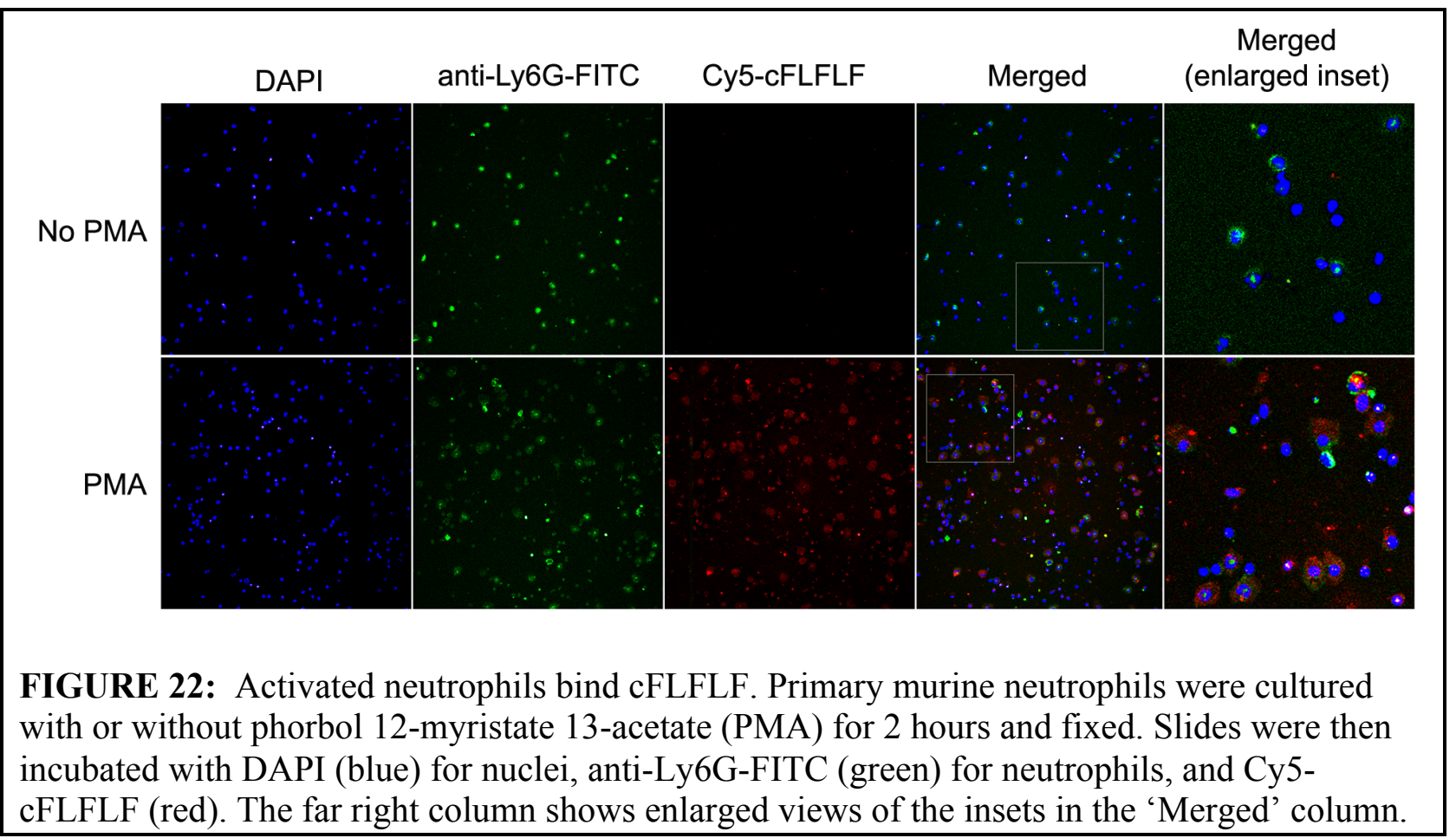




\section{Cy7-cFLFLF Signal Correlates with Neutrophils}

Immunofluorescence staining of lung tissue sections after IR 2-hr demonstrated that increased neutrophil activation and infiltration after IRI corresponds with increased Cy7-cFLFLF signal, compared with sham lung tissue that demonstrated few neutrophils and minimal Cy7cFLFLF signal (Figure 23).

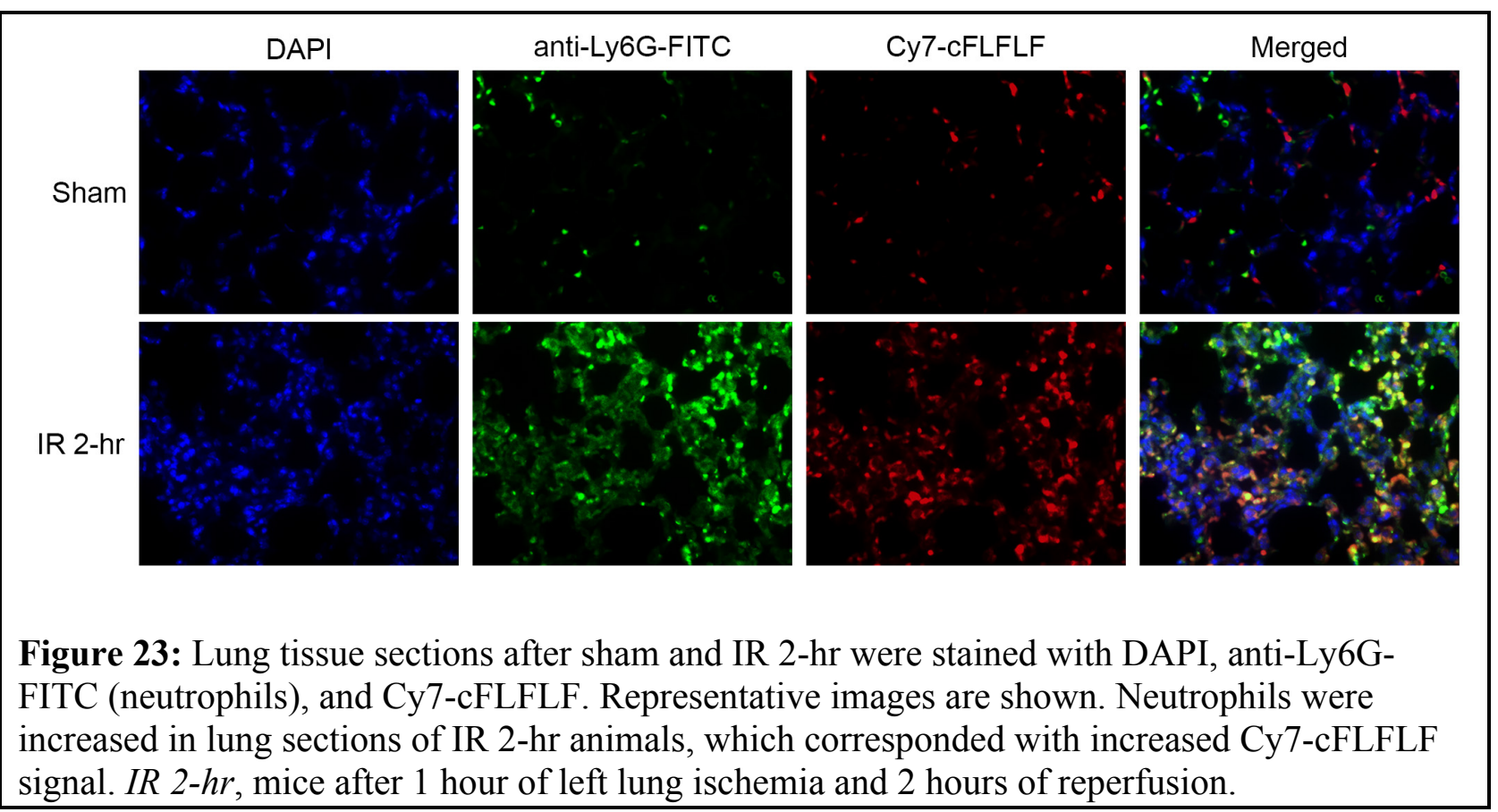

\section{Discussion}

The present study investigated the diagnostic utility of in vivo leukocyte labeling with an FPR1-specific ligand (cFLFLF) to noninvasively diagnose and monitor lung IRI. Injection of ${ }^{99 \mathrm{~m}} \mathrm{Tc}$-cFLFLF allowed for visualization of leukocyte activity after IR via SPECT imaging. ${ }^{99 \mathrm{~m}} \mathrm{Tc}$-cFLFLF uptake in injured lungs and SPECT signal intensity correlated with changes in pulmonary function over time, allowing resolution of injury to be monitored. Changes in pulmonary function (compliance, airway resistance, and PA pressure) demonstrated peak lung injury after 2 hours of reperfusion, with lung function improving over 12 and 24 hours of 
reperfusion. Neutrophil infiltration was correlated with pulmonary dysfunction and SPECT/CT imaging results, with peak neutrophil infiltration occurring after 2 hours of reperfusion. Organ biodistribution revealed significant differences in ${ }^{99 \mathrm{~m}} \mathrm{Tc}-\mathrm{cFLFLF}$ uptake in injured left lungs compared with sham animals. The specificity of cFLFLF to allow for SPECT imaging of left lung IRI was confirmed using a Cy7-labeled cFLFLF and near infrared fluorescence imaging.

Lung transplantation is often a life-saving operation for patients with end-stage lung disease, but successful outcomes are less common compared to other solid organ transplants. [14] The risk of lung IRI leading to PGD in the immediate postoperative period drives transplant surgeons to be conservative in their acceptance of donor organs. [19] Unfortunately, mortality for patients on the lung transplant waiting list continues to rise as the demand for organs far outweighs the supply of "acceptable" organs. [3] When PGD occurs, early morbidity and mortality rises, as does the rate of long-term complications such as bronchiolitis obliterans. [1316] Transplant clinicians are limited in their ability to diagnose and monitor PGD, having to rely mainly on nonspecific chest radiographs and arterial oxygenation values.

Providing clinicians with the ability to noninvasively image active inflammation with cell-specific molecular probes may allow for earlier diagnosis, earlier initiation of targeted therapies, and a method to monitor resolution or progression of PGD. Lung IRI is a complex inflammatory process that entails rapid activation of proinflammatory resident alveolar macrophages and iNKT cells. [22, 25] Subsequent release of HMGB1, TNF- $\alpha$, IL-1 $\beta$, and IL-17 leads to rapid chemokine production (e.g. IL-8 from epithelium), neutrophil recruitment and a significant ramping up of the inflammatory process. [21, 82] Activated neutrophils express FPR1, a chemotactic G-protein-coupled surface receptor that binds mitochondrial N-formyl peptides, which are known damage-associated molecular patterns (DAMPs). [76, 83] Our 
laboratory developed a cFLFLF as a ligand with high binding affinity for FPR1 and allows for noninvasive in vivo imaging of leukocytes, predominantly activated PMNs, during acute inflammation. [74] As seen in Figure 23, increased cFLFLF signal corresponds with neutrophil infiltration in lung tissue after IRI, but that cFLFLF also binds other cells expressing FPR1. Macrophages are known to express FPR1 and we have evidence that macrophages bind cFLFLF, therefore we hypothesize that the additional ${ }^{99 \mathrm{~m}} \mathrm{Tc}$-cFLFLF signal seen in our model is likely from the presence of macrophages. $[84,85]$

The present study demonstrated the feasibility of using ${ }^{99 \mathrm{~m}}$ Tc-cFLFLF to label activated PMNs in vivo allowing us to image sites of active inflammation with SPECT. Using a mouse model of lung IRI that showed peak injury at 2 hours, with resolution over time out to 24 hours, SPECT imaging results showed a 4-fold difference in left lung signal intensity at 2 hours, a 2fold difference at 12-hours, and a non-significant difference at 24 hours (which correlated with improvement in lung function and reduced neutrophil counts) compared with sham animals.

Conjugation of cFLFLF with various isotopes and fluorescence dyes allows for visualization of the probe with different imaging modalities, such as positron emission tomography (PET), SPECT, optical imaging, and MRI. [74, 75, 77, 86] Regardless of probe label and imaging modality, cFLFLF is quite specific for detection of leukocyte-mediated inflammation. In a rat model of acute osteomyelitis, ${ }^{99 \mathrm{~m}}$ Tc-labeled cFLFLF with SPECT imaging was found to be superior to ${ }^{99 \mathrm{~m}} \mathrm{Tc}-\mathrm{methylene}$ diphosphonate bone scanning and ${ }^{18} \mathrm{~F}$ fluorodeoxyglucose (FDG) PET/CT for diagnosis based on specificity and image quality, and that ${ }^{99 \mathrm{~m}} \mathrm{Tc}-\mathrm{cFLFLF}$ was effective at evaluating the therapeutic response to treatment. [87] The results of our current study support these findings. ${ }^{99 \mathrm{~m}} \mathrm{Tc}-\mathrm{cFLFLF}$ is a useful imaging agent that can be applied broadly to different disease processes involving acute inflammation and appears 
to be more specific than other nuclear medicine imaging techniques. Although ${ }^{18} \mathrm{~F}-\mathrm{FDG}-\mathrm{PET}$ is used extensively to evaluate primary and recurrent forms of cancer, including breast, head/neck, and lung, it has not been shown to be particularly effective for other forms of inflammation. [8890] Metabolically active cells, especially tumor cells, take up ${ }^{18}$ F-FDG, and thus ${ }^{18}$ F-FDG does not allow for delineation of specific cell-types, which limits its utility for accurate diagnosis of inflammatory processes such as lung IRI.

A consistent method of labeling activated leukocytes in vivo would be a significant breakthrough for the field of nuclear medicine as most successful methods of leukocyte labeling thus far have required ex vivo radiolabeling. [18] Although currently the gold standard for radionuclide imaging of inflammation and infection, ex vivo methods are labor-intensive and have a high risk of contamination, requiring blood or bone marrow extraction, ex vivo leukocyte labeling, and injection of the cells back into the patient. Given these limitations, attempts have been made using antigranulocyte antibodies for in vivo leukocyte labeling, but there has been limited success. Agents that have been investigated include a monoclonal IgG1 antibody to the NCA-95 antigen on leukocytes (BW 250/183, Granuloscint; CISBio International, Yvette, France), a fragment of a monoclonal antibody that binds NCA-90 antigen (Sulesomab, Leukoscan, Immunomedics, Morris Plains, NJ), and ${ }^{99 \mathrm{~m}}$ Tc-Fanolesomab (NeutroSpec, Palatin Technologies, Cranbury, NJ), an IgM antibody that binds CD15. [91-94] Varying sensitivities and specificities have limited the adoption of these agents into clinical practice.

The present study demonstrates the effectiveness of using ${ }^{99 \mathrm{~m}}$ Tc-cFLFLF to label leukocytes in vivo for the noninvasive diagnosis and monitoring of lung IRI by SPECT. Our study is limited by the fact that cFLFLF only targets FPR1 positive leukocytes; largely PMNs and possibly macrophages. $[75,76]$ Although leukocyte infiltration is an important component of 
lung IRI, the ability to noninvasively image the activation of all major cell populations involved (including non-immune cells) may be needed for this diagnostic modality to positively impact lung transplantation outcomes. For example, development and implementation of specific M1 and M2 macrophage probes would further enhance our ability to noninvasively characterize the inflammatory state of transplanted lungs and to monitor resolution of injury. The ability to monitor changes in proinflammatory cell populations, such as M1 macrophages and infiltrating PMNs, as compared with anti-inflammatory cell populations, such as M2 macrophages, may allow for an accurate understanding of the different stages of lung IRI and PGD, and allow clinicians to intervene with appropriate treatments. Additionally, since our model is a hilar clamp IR model, the findings need to be confirmed in a mouse or large animal lung transplant model. In conclusion, using a reproducible murine model of left lung IRI, the present study shows that administration of ${ }^{99 \mathrm{~m}}$ Tc-cFLFLF permits quantitative, noninvasive diagnosis of IRI via SPECT imaging. Importantly, changes in ${ }^{99 \mathrm{~m}}$ Tc-cFLFLF uptake and SPECT signal intensity correlated with changes in lung function parameters over time, suggesting that resolution of IRI over time can be monitored with ${ }^{99 \mathrm{~m}} \mathrm{Tc}-\mathrm{cFLFLF}$ and SPECT imaging. In vivo leukocyte labeling with cell-specific molecular imaging probes such as cFLFLF may allow for earlier and more accurate diagnosis of IRI, early initiation of targeted therapeutic interventions, and improved outcomes after lung transplantation. 


\section{Molecular Targets to Attenuate Lung IR Injury}

Three molecular targets for pharmacologic therapies are investigated. In a preclinical porcine model of lung transplantation, enhanced EVLP with adenosine A2B receptor antagonist is tested. The role of TRPV4 receptors during lung IRI as an additional target is explored. Panx1 endothelial cell signaling mechanisms and Panx1 antagonism are evaluated as a novel therapeutic strategy to prevent lung IRI. 


\section{PROJECT \#4}

\section{Lungs Donated After Circulatory Death and Prolonged Warm Ischemia Are Successfully Transplanted After Enhanced Ex Vivo Lung Perfusion Using Adenosine A2B Receptor Antagonism}

Eric J. Charles MD ${ }^{1}$, J Hunter Mehaffey $\mathrm{MD}^{1}$, Ashish K Sharma MBBS PhD ${ }^{1}$, Yunge Zhao MD PhD ${ }^{1}$, Mark H Stoler $\mathrm{MD}^{2}$, James M Isbell MD MSCI ${ }^{1}$,

Christine L Lau MD MBA ${ }^{1}$, Curtis G Tribble MD, ${ }^{1}$ Victor E Laubach $\mathrm{PhD}^{1}$, Irving L Kron MD ${ }^{1}$

Departments of ${ }^{1}$ Surgery and ${ }^{2}$ Pathology, University of Virginia, Charlottesville, Virginia

\section{Status of Research: PUBLISHED}

Charles EJ, et al. Journal of Thoracic and Cardiovascular Surgery. Apr 12, 2017;

[Epub ahead of print]. PMID: 28483262 


\section{Abstract}

Background: The current supply of acceptable donor lungs is not sufficient for the number of patients awaiting transplantation. We hypothesized that ex vivo lung perfusion (EVLP) with targeted drug therapy would allow successful rehabilitation and transplantation of donation after circulatory death (DCD) lungs exposed to 2-hours of warm ischemia.

Methods: Donor porcine lungs were procured after 2-hours of warm ischemia post-cardiac arrest, and subjected to 4-hours of cold preservation or EVLP. ATL802, an adenosine $A_{2 B}$ receptor antagonist, was administered to select groups. Four groups $(n=4 /$ group $)$ were randomized: cold preservation (Cold), cold preservation with ATL802 during reperfusion (Cold+ATL802), EVLP (EVLP), and EVLP with ATL802 during ex vivo perfusion (EVLP+ATL802). Lungs were subsequently transplanted, reperfused, and assessed by measuring dynamic lung compliance and oxygenation capacity.

Results: EVLP+ATL802 significantly improved dynamic lung compliance compared with EVLP $\left(25.0 \pm 1.8\right.$ vs $\left.17.0 \pm 2.4 \mathrm{~mL} / \mathrm{cmH}_{2} \mathrm{O}, \mathrm{p}=0.04\right)$, and compared with cold preservation (Cold: 12.2 $\pm 1.3, \mathrm{p}=0.004 ;$ Cold+ATL802: $\left.10.6 \pm 2.0 \mathrm{~mL} / \mathrm{cmH}_{2} \mathrm{O}, \mathrm{p}=0.002\right)$. Oxygenation capacity was highest in EVLP (440.4 \pm 37.0 vs Cold: $174.0 \pm 61.3 \mathrm{mmHg}, \mathrm{p}=0.037)$. No differences in oxygenation or pulmonary edema were observed between EVLP and EVLP+ATL802. A significant decrease in IL-12 expression in tissue and bronchoalveolar lavage was identified between groups EVLP and EVLP+ATL802, along with less neutrophil infiltration.

Conclusions: Severely injured DCD lungs subjected to 2-hours of warm ischemia are successfully transplanted after enhanced EVLP with targeted drug therapy. Increased utilization of lungs following uncontrolled donor cardiac death and prolonged warm ischemia may be possible and may improve transplant wait list times and mortality. 


\section{Introduction}

Lung transplantation is a life-saving operation for patients with end-stage lung disease, but is accompanied by significant hurdles that must be overcome to ensure a positive result. Central to the discussion is the shortage of acceptable donor lungs, given the conservative acceptance criteria used by most surgeons and the increased risk of primary graft dysfunction (PGD) with transplantation of marginal lungs. [19] Considering outcomes after lung transplantation are the worst of any solid organ, strategies to optimize lung utilization and increase the likelihood of a successful outcome are needed. [3]

The expansion of donor acceptance criteria to include donation after circulatory death (DCD) lungs is one such approach that may substantially increase the pool of available organs. [42] Currently, DCD lungs are used in less than $2 \%$ of lung transplants per year due to the increased risk of ischemia-reperfusion (IR) injury, a major cause of PGD and a risk factor for the development of chronic graft rejection. $[3,95]$ The use of ex vivo lung perfusion (EVLP) prior to transplantation may help alleviate the resultant IR injury. [17] Using EVLP to perform donor lung assessment followed by targeted therapeutic rehabilitation may allow for successful transplantation of otherwise unacceptable lungs.

Targeting various cellular receptors in the lung, such as adenosine and sphingosine-1phosphate receptors, our laboratory has shown improved outcomes and attenuation of IR injury after transplantation. [41, 57] Adenosine, a bioactive nucleoside, plays a significant role in purinergic signaling during inflammation through its interaction with four G-protein-coupled receptors $\left(\mathrm{A}_{1}, \mathrm{~A}_{2 \mathrm{~A}}, \mathrm{~A}_{2 \mathrm{~B}}, \mathrm{~A}_{3}\right)$. [96] Altering the level of adenosine receptor signaling with targeted drug delivery during EVLP may help improve outcomes after lung transplantation. The role of adenosine $\mathrm{A}_{2 \mathrm{~B}}$ receptor $\left(\mathrm{A}_{2 \mathrm{~B}} \mathrm{R}\right)$ activation has been found to be both pro-inflammatory and anti- 
inflammatory in different experimental models of lung injury. [48, 97-99] Our laboratory demonstrated that it is pro-inflammatory and that selective antagonism of $\mathrm{A}_{2 \mathrm{~B}} \mathrm{R}$ attenuates IR injury. [100]

The objective of the current study was to determine if lungs procured following donor cardiac death and prolonged warm ischemia could be rehabilitated with EVLP and successfully transplanted, using a porcine model of lung transplantation. We hypothesized that use of EVLP with addition of an $\mathrm{A}_{2 \mathrm{~B}} \mathrm{R}$ antagonist, as targeted drug therapy during the 4-hour ex vivo perfusion period, would allow for successful rehabilitation and transplantation of DCD lungs exposed to 2hours of warm ischemia. The use of lungs for transplantation following uncontrolled donor cardiac death in the field could increase the pool of available organs, increase the number of lung transplants per year, and decrease wait list times and mortality.

\section{Materials and Methods}

Animals and Study Groups

This study complied with the 1996 Guide for the Care and Use of Laboratory Animals as recommended by the US National Institutes of Health. The University of Virginia Animal Care and Use Committee approved the study protocol and all animals received humane care.

Mature domestic swine of both sexes (19-42 kg) underwent hypoxic cardiac arrest, followed by 2-hours of warm ischemia prior to cold preservation flush and procurement of the lungs. Two hours of warm ischemia time reflects the estimated amount of time necessary to procure lungs following uncontrolled donor cardiac death in the field. Donor lungs were randomized to four groups ( $\mathrm{n}=4$ /group): 4-hours of cold preservation at $4^{\circ} \mathrm{C}$ (Cold), 4-hours of cold preservation at $4{ }^{\circ} \mathrm{C}$ with the addition of ATL802 during the reperfusion period 
(Cold+ATL802), 4-hours of normothermic EVLP with Steen Solution ${ }^{\mathrm{TM}}$ (XVIVO Perfusion Inc, Englewood, CO) (EVLP), or 4-hours of EVLP supplemented with ATL802 (EVLP+ATL802)

(Figure 24). In all groups, the left lung was subsequently transplanted into a size-matched recipient and reperfused for 4 hours in vivo. ATL802 (Lewis and Clark Pharmaceuticals, Charlottesville, VA), a selective $A_{2 B} R$ antagonist, was given to groups Cold+ATL802 (via external jugular vein at start of reperfusion period) and EVLP+ATL802 (added to Steen at start of ex vivo perfusion period) as targeted anti-inflammatory drug therapy at a bolus dose of 1 $\mathrm{mg} / \mathrm{kg}$, based on previous studies. [100, 101] ATL802 is currently being used for preclinical studies only to determine the efficacy of $\mathrm{A}_{2 \mathrm{~B}} \mathrm{R}$ antagonism.

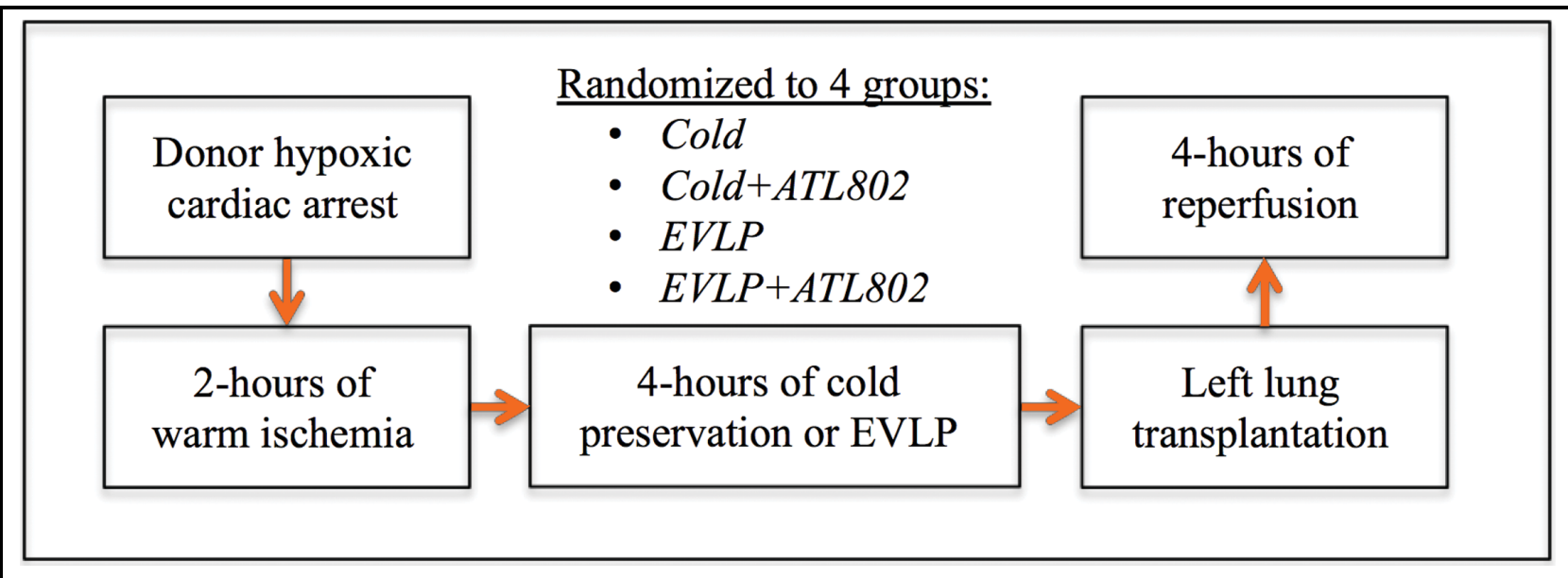

Figure 24: Schematic of experiment. Cold, transplantation after 4-hours of cold preservation; Cold + ATL802, transplantation after 4-hours of cold preservation with administration of ATL802 during reperfusion; $E V L P$, transplantation after 4-hours of EVLP; EVLP $+A T L 802$, transplantation after 4-hours of EVLP with administration of ATL802 during ex vivo perfusion.

\section{Donor Procurement Procedure}

Procurement of donor lungs was performed as previously described. [45] Ketamine (50 $\mathrm{mg} / \mathrm{kg}$ ) and xylazine $(5 \mathrm{mg} / \mathrm{kg})$ were used for induction of anesthesia and intubation. Isoflurane $(3 \%)$ and 1.0 fraction of inspired oxygen $\left(\mathrm{FiO}_{2}\right)$ were used to maintain anesthesia. Animals were ventilated (tidal volume $8 \mathrm{~mL} / \mathrm{kg}$, respiratory rate 16-20 breaths/min, positive end-expiratory 
pressure $5 \mathrm{cmH}_{2} \mathrm{O}$ ) and an initial arterial blood gas (ABG) sample was analyzed. All donor animals received systemic heparin (200 U/kg, Hospira Inc., Lake Forest, IL) and had continuous electrocardiogram monitoring in place prior to clamping the endotracheal tube and inducing hypoxic cardiac arrest. Donor animals underwent 2-hours of warm ischemia from the time asystole was confirmed on the cardiac monitor.

A median sternotomy was then performed and a cardioplegia cannula (Terumo Heart Inc., Ann Arbor, MI) was inserted into the main pulmonary artery (PA) to deliver ProstaglandinE1 (500 $\mu$ g, Pfizer Inc., New York, NY) and cold Perfadex ${ }^{\circledR}$ (XVIVO Perfusion Inc., Englewood, CO). A total of 1.5 liters of Perfadex supplemented with 15,000 IU of heparin was flushed through the lungs after venting the left atrial (LA) appendage and ligating the vena cava. Ice slush was introduced into the thorax to rapidly cool the lungs. The trachea was clamped midinspiration and the heart-lung bloc was explanted.

For groups Cold and Cold+ATL802, the heart and right-lung were removed on the backtable and preparation of the left bronchus, PA, and LA cuff was completed. Retrograde flush with an additional $500 \mathrm{~mL}$ of cold Perfadex with heparin was completed and the lungs were placed in a protective plastic bag and stored at $4^{\circ} \mathrm{C}$ for 4-hours. For groups EVLP and EVLP+ATL802, the heart was removed on the back-table and the trachea, main PA, and LA cuff were prepared to allow for placement of the EVLP cannulas.

\section{EVLP}

Lungs randomized to groups EVLP and EVLP+ATL802 underwent 4-hours of normothermic EVLP. [46, 47] A yellow cannula (XVIVO Perfusion Inc., Englewood, CO) was placed in the main PA, a green cannula (XVIVO Perfusion Inc., Englewood, CO) was sutured to 
the LA cuff, and a 7-0 endotracheal tube was placed into the trachea. An additional $500 \mathrm{~mL}$ of cold Perfadex was flushed retrograde.

EVLP was initiated on both lungs as previously described. [38] The circuit was primed with 2L Steen supplemented with cefazolin (500 mg, APP Pharmaceuticals, Schaumburg, IL), methylprednisolone (500 mg, Pfizer Inc., New York, NY), and heparin (10,000 IU). ATL802 was added to the ex vivo perfusate for lungs randomized to EVLP+ATL802 and dimethyl sulfoxide (vehicle) was added for group EVLP. Surgeons were blinded as to whether the perfusate was supplemented with the treatment drug or vehicle. Flow was initiated $(0.2 \mathrm{~mL} / \mathrm{min})$ and LA pressures maintained between $0-5 \mathrm{mmHg}$. The perfusate was warmed to $37^{\circ} \mathrm{C}$ over 30 minutes. Flow was titrated up to $40 \%$ of estimated cardiac output $(100 \mathrm{~mL} / \mathrm{kg}$ donor body weight) and when the perfusate reached $32^{\circ} \mathrm{C}$, ventilation was started (tidal volume $8 \mathrm{~mL} / \mathrm{kg}$,

respiratory rate 8 breaths/minute, positive end-expiratory pressure $5.0 \mathrm{cmH}_{2} \mathrm{O}, \mathrm{FiO}_{2} \mathrm{0.21}$ ). A trigas mixture ( $86 \%$ nitrogen, $8 \%$ carbon dioxide, $6 \%$ oxygen) was used to deoxygenate the perfusate.

Samples from the PA inflow and LA outflow were collected hourly following a 15minute challenge with $1.0 \mathrm{FiO}_{2}$ to measure the partial pressure of oxygen $\left(\mathrm{PaO}_{2}\right)$. Airway pressures were measured hourly and used to calculate dynamic compliance. After 4-hours of EVLP, lungs were flushed anterograde with cold Perfadex $(500 \mathrm{~mL})$ and the left lung was separated off and prepared for transplantation.

\section{Lung Transplant Procedure}

Recipient animals were anesthetized and ventilated similar to donors. A multi-access cathether with PA cathether and an arterial catheter were placed. The animal was administered 
lidocaine $(50 \mathrm{mg}$ ) and heparin (5000 IU). The transplant procedure was performed as previously described: left lateral thoracotomy, left pneumonectomy, left lung transplant (running sutures for anastomoses: end-to-end bronchial, end-to-end PA, and LA cuff to recipient LA appendage). [57] At the start of reperfusion, ATL802 was administered via external jugular vein injection for lungs randomized to group Cold+ATL802.

\section{Post-transplant Reperfusion}

In vivo reperfusion was maintained for 4-hours. Airway pressure measurements and systemic ABGs were performed every 30-minutes. Low-pressure lung recruitment was performed prior to each set of measurements. Superior and inferior pulmonary vein blood samples were obtained after 2-hours and at the completion of 4-hours of reperfusion for direct left lung $\mathrm{PaO}_{2}$ measurements. Adequate hemodynamics and acid/base status were maintained with use of normal saline, epinephrine, and sodium bicarbonate to meet the following goals: $\mathrm{pH}$ 7.35-7.45, base excess $>-5$, and mean arterial pressure $>55 \mathrm{mmHg}$. The lung was explanted after 4-hours of reperfusion and the animal euthanized.

\section{Pulmonary Edema}

Left lungs were weighed just prior to transplant and immediately after explant. Percent gross weight change from pre-transplantation to post-reperfusion was calculated to determine amount of pulmonary edema.

Cytokine Measurements

After explantation, two fresh tissue samples were obtained (upper and lower portion of lower lobe), flash frozen in liquid nitrogen, and stored at $-80^{\circ} \mathrm{C}$. FastPrep ${ }^{\circledR}-24$ (MP 
Biomedicals, Santa Ana, CA) was used to homogenize tissue. Bicinchoninic acid protein assay (Pierce, Rockford, IL) was used to determine total protein concentration in the supernatant of each homogenized tissue sample. Bronchoalveolar lavage (BAL) of the upper lobe was performed with $30 \mathrm{~mL}$ of normal saline, centrifuged, and stored at $-80^{\circ} \mathrm{C}$. A commercially available multiplex immunosorbent assay (EMD Millipore, Billerica, MA) was used to measure levels of interleukin (IL)-1 $\alpha, 1 \beta, 4,6,8,10,12,18$, and TNF- $\alpha$ in tissue supernatant (normalized to equal protein concentrations) and BAL.

\section{Histopathologic Assessment}

The lower lobe was instilled with $10 \%$ buffered formalin via the bronchus after all fresh tissue sampling was complete, and then submerged in formalin. After storage overnight, peripheral lung tissue samples ( $\mathrm{n}=4 / \mathrm{lung}$ ) were obtained, paraffin-embedded and sectioned. One histology slide from each sample was stained with hematoxylin-eosin and two histology slides were used for immunohistochemistry evaluation of neutrophil infiltration.

The H\&E stained slides were assessed by a masked pathologist for presence of lung injury. Each slide was scored based on the following three components: polymorphonuclear cells per 40X high-powered field (HPF) $(0=<5,1=6-10,2=11-20,3=>20)$, alveolar edema $(0=<5 \%, 1=6-25 \%, 2=26-50 \%, 3=>50 \%)$, and interstitial inflammation $(0=$ none, $1=$ minimal, $2=$ moderate, $3=$ severe), as previously described. [57]

For neutrophil immunohistochemistry staining, the primary antibody used was mouse monoclonal anti-porcine neutrophil antibody (MBA Biomedicals, Augst, Switzerland) and secondary antibody used was donkey anti-mouse IgG (Jackson ImmunoResearch Laboratories Inc., West Grove, PA). After addition of avidin-biotin complex, slides were incubated for 30- 
minutes at room temperature, followed by incubation with 3,3-diaminobenzidine tetrahydrochloride (Dako Inc., Carpinteria, CA) to produce a brown precipitate, and finally counterstained with hematoxylin. [48] Five microscopic photographs of each slide were taken at 40X magnification. The number of neutrophils per HPF was counted by a masked reviewer and averaged per tissue sample to account for potential injury heterogeneity.

\section{Statistical Analysis}

One-way analysis of variance with Bonferroni's multiple comparisons test and Student's t-test were used to determine statistical significance between groups. Prism 6 (GraphPad Software Inc., La Jolla, CA) was used to perform statistical calculations and all data were reported as mean \pm standard error of the mean, with p-value for significance of 0.05 .

\section{Results}

Lung Function

After transplantation and 4-hours of in vivo reperfusion, dynamic compliance was significantly higher in group EVLP+ATL802 compared with the vehicle EVLP group (25.0 1.8 vs. $17.0 \pm 2.4 \mathrm{~mL} / \mathrm{cmH}_{2} \mathrm{O}, \mathrm{p}=0.04$ ), and compared to both non-EVLP groups (Cold: $12.2 \pm 1.3$, $\mathrm{p}=0.004$ and Cold $\left.+\mathrm{ATL} 802: 10.6 \pm 2.0 \mathrm{~mL} / \mathrm{cmH}_{2} \mathrm{O}, \mathrm{p}=0.002\right)($ Figure 25A). Oxygenation capacity of the transplanted lung after 4-hours of reperfusion was highest in group EVLP (440.4 $\pm 37.0 \mathrm{mmHg}$ ) and significant compared to Cold $(174.0 \pm 61.3 \mathrm{mmHg}, \mathrm{p}=0.037)$ (Figure 25B). There were no significant differences in final $\mathrm{PaO}_{2} / \mathrm{FiO}_{2}$ ratios between groups Cold, Cold+ATL802, and EVLP+ATL802. 


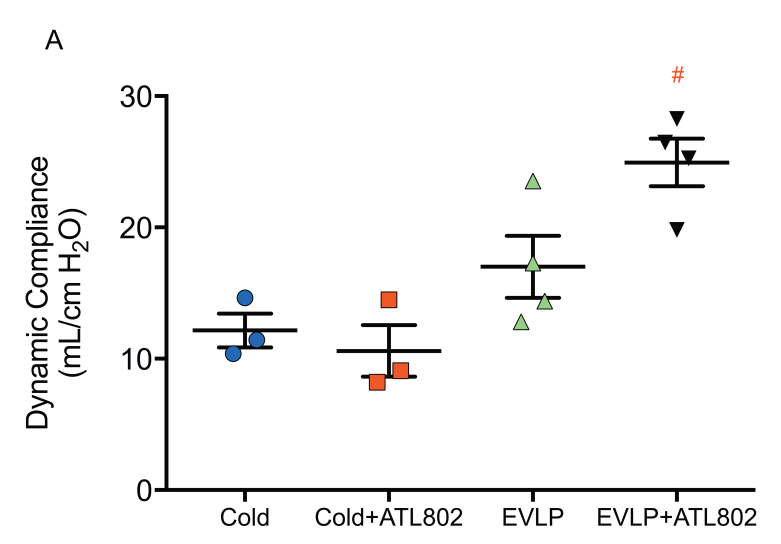

B

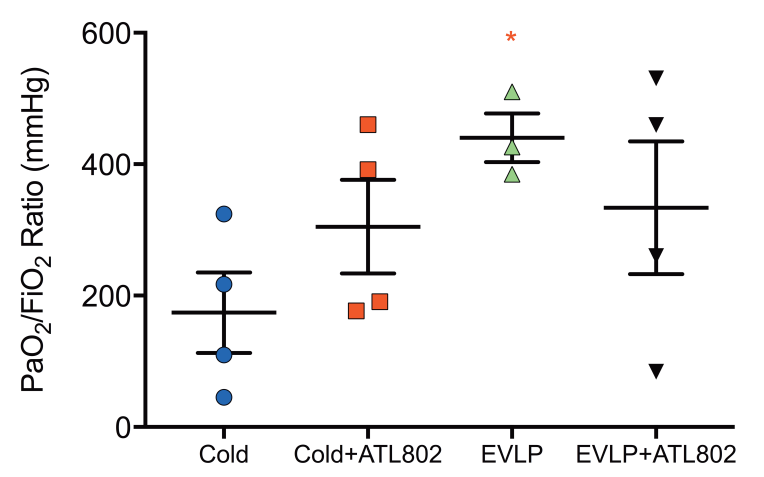

Figure 25: (A) Final dynamic compliance and (B) oxygenation $\left(\mathrm{PaO}_{2} / \mathrm{FiO}_{2}\right)$ at the completion of 4-hours of reperfusion. Cold, transplantation after 4-hours of cold preservation; Cold +ATL802, transplantation after 4-hours of cold preservation with administration of ATL802 during reperfusion; $E V L P$, transplantation after 4-hours of EVLP; EVLP+ATL802, transplantation after 4-hours of EVLP with administration of ATL802 during ex vivo perfusion; $\mathrm{PaO} 2 / \mathrm{FiO}$, partial pressure of oxygen/fraction of inspired oxygen. $\# \mathrm{p}=0.04$ vs EVLP, $p=0.002$ vs Cold+ATL802, and $\mathrm{p}=0.004$ vs Cold, ${ }^{*} \mathrm{p}=0.037$ vs Cold.

Prior to inducing hypoxic cardiac arrest, donor animals from all groups had similar $\mathrm{PaO}_{2} / \mathrm{FiO}_{2}$ ratios (Cold: 501.7 \pm 28.9 , Cold+ATL802: 510.2 \pm 29.9, EVLP: $454.8 \pm 17.0$, EVLP+ATL802: $490.3 \pm 39.8 \mathrm{mmHg}, \mathrm{p}=0.59)$ with an overall mean donor $\mathrm{PaO}_{2} / \mathrm{FiO}_{2}$ ratio of 489.3 $\pm 14.5 \mathrm{mmHg}$. After clamping the endotracheal tube, the mean time to death for all donor animals was $22.6 \pm 2.0$ minutes, with no difference between groups $(\mathrm{p}=0.2)$.

During the 4-hour EVLP period, no significant differences in oxygenation capacity or dynamic compliance were observed between groups EVLP and EVLP+ATL802, although the hourly mean values for both parameters were consistently higher in EVLP+ATL802 (Figure 26). By hour 1 of EVLP, the mean $\mathrm{PaO}_{2} / \mathrm{FiO}_{2}$ ratios for both groups were above $300 \mathrm{mmHg}$ (EVLP: 375 \pm 23.3, EVLP+ATL802: $427.2 \pm 83.6 \mathrm{mmHg}$ ) and remained elevated at the completion of 4-

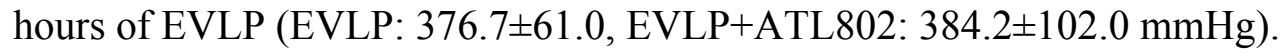



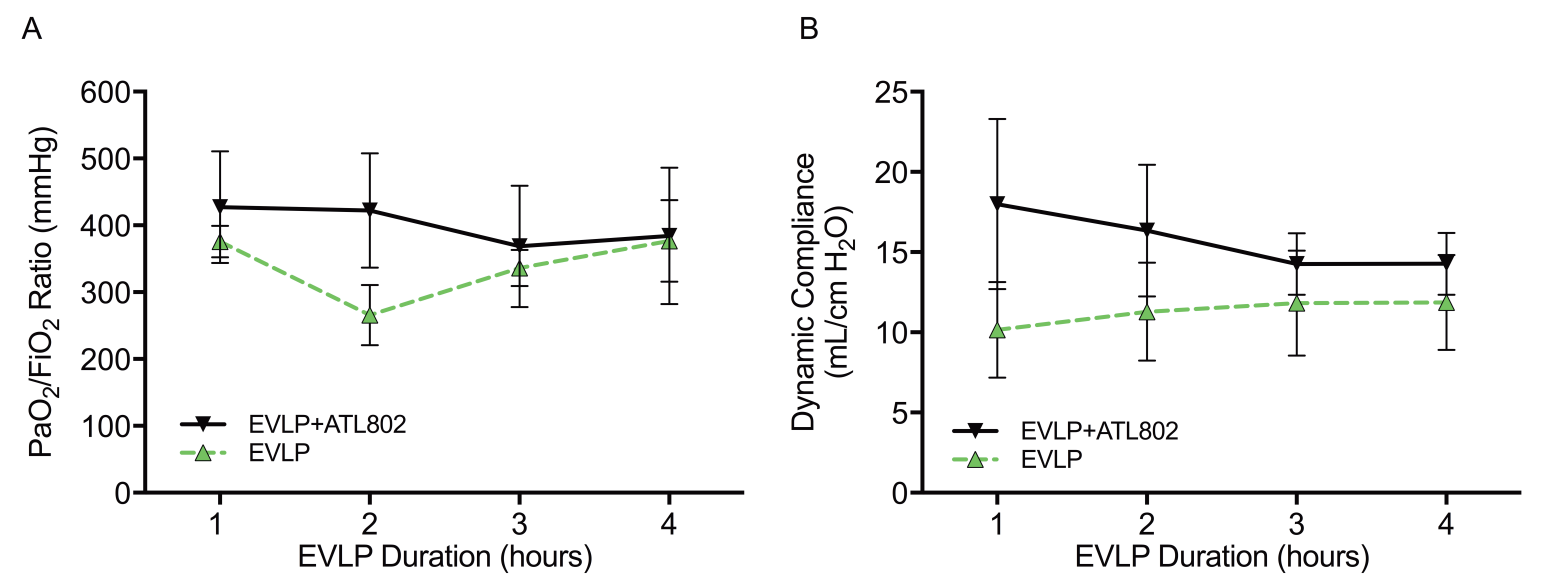

Figure 26: (A) Oxygenation $\left(\mathrm{PaO}_{2} / \mathrm{FiO}_{2}\right)$ and (B) dynamic compliance recorded hourly during EVLP. Cold, transplantation after 4-hours of cold preservation; Cold + ATL802, transplantation after 4-hours of cold preservation with administration of ATL802 during reperfusion; EVLP, transplantation after 4-hours of EVLP; $E V L P+A T L 802$, transplantation after 4-hours of EVLP with administration of ATL802 during ex vivo perfusion; $\mathrm{PaO} 2 / \mathrm{FiO} 2$, partial pressure of oxygen/fraction of inspired oxygen.

The mean $\mathrm{PaO}_{2} / \mathrm{FiO}_{2}$ ratios from the start of the experiment to conclusion of the 4-hour reperfusion period for the two untreated groups (Cold and EVLP) are shown in Figure 27. The Cold group decreased significantly from an initial donor $\mathrm{PaO}_{2} / \mathrm{FiO}_{2}$ ratio of $501.7 \pm 28.9 \mathrm{mmHg}$ to a post-reperfusion mean of $174.0 \pm 61.3 \mathrm{mmHg}(\mathrm{p}=0.003)$, while the final post-reperfusion $\mathrm{PaO}_{2} / \mathrm{FiO}_{2}$ ratio for the EVLP group was not significantly different compared with the donor prehypoxia ratio $(440.4 \pm 37.0$ vs. $454.8 \pm 17.0, \mathrm{p}=0.71)$.

\section{Hemodynamic Support}

During the reperfusion period, intravenous normal saline, epinephrine, and sodium bicarbonate were used judiciously to maintain adequate hemodynamics and appropriate acid/base status. Recipient animals in EVLP+ATL802 were the most stable, requiring the least amount of 
fluids $(0.7 \pm 0.1 \mathrm{~L})$, epinephrine $(1.2 \pm 0.4 \mathrm{mg})$, and sodium bicarbonate $(1.0 \pm 0.0 \mathrm{amps})$ during the reperfusion period (Table 3).

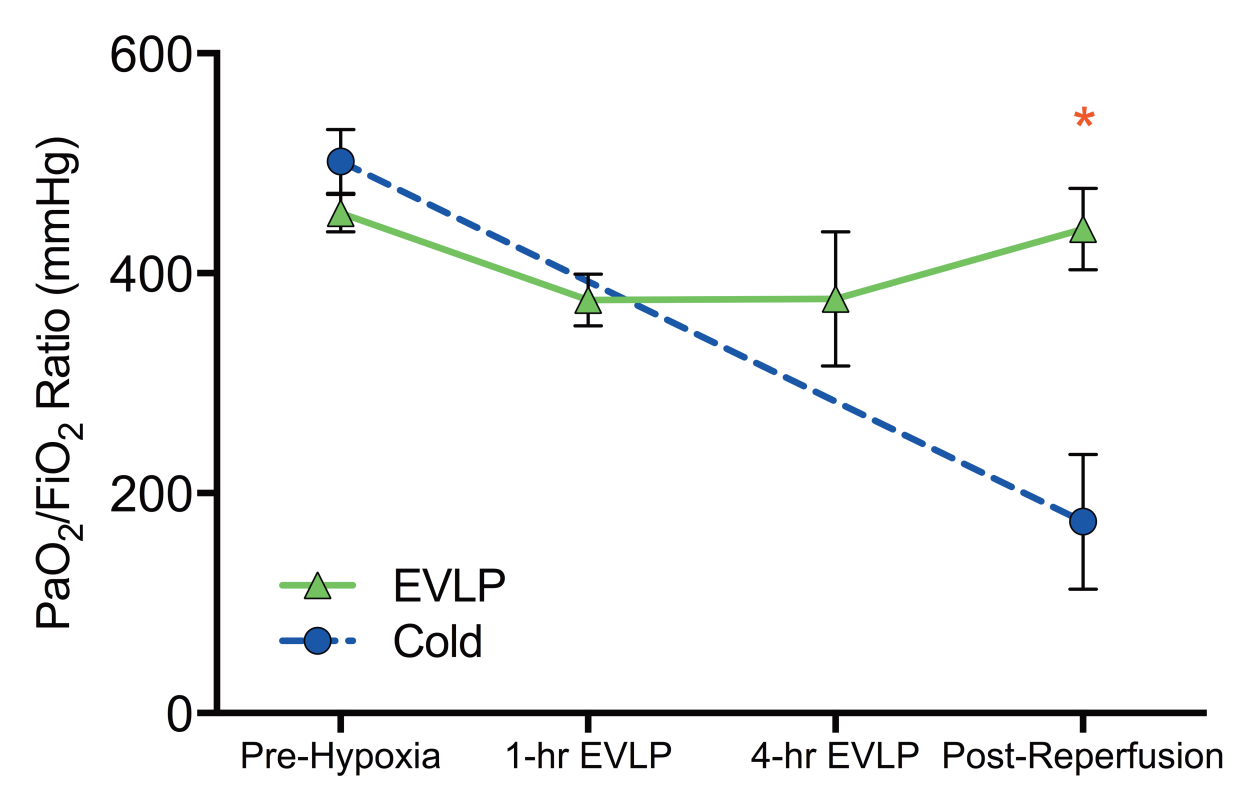

Figure 27: Oxygenation capacity $\left(\mathrm{PaO}_{2} / \mathrm{FiO}_{2}\right)$ of groups without drug treatment recorded at four time points: donor animal prior to hypoxia (Pre-hypoxia), 1-hour into EVLP (1-hour EVLP), at the completion of EVLP (4-hour EVLP), and recipient after 4-hours of reperfusion (PostReperfusion). Cold, transplantation after 4-hours of cold preservation; EVLP, transplantation after 4-hours of EVLP; $\mathrm{PaO} / \mathrm{FiO}$, partial pressure of oxygen/fraction of inspired oxygen. * $\mathrm{p}=0.037$.

Table 3: Intravenous fluid, epinephrine, and sodium bicarbonate requirements during 4hour reperfusion period (mean $\pm \mathrm{SE}$ )

\begin{tabular}{|l|c|c|c|}
\hline & Intravenous Fluid (L) & Epinephrine (mg) & Sodium Bicarbonate (amp) \\
\hline Cold & $1.2 \pm 0.3$ & $7.9 \pm 4.2$ & $1.0 \pm 0.4$ \\
\hline Cold+ATL802 & $2.2 \pm 0.6$ & $14.1 \pm 6.3$ & $3.5 \pm 1.0$ \\
\hline EVLP & $1.0 \pm 0.3$ & $2.1 \pm 0.3$ & $0.8 \pm 0.5$ \\
\hline EVLP+ATL802 & $0.7 \pm 0.1$ & $1.2 \pm 0.4$ & $1.0 \pm 0.0$ \\
\hline
\end{tabular}

Cold, transplantation after 4-hours of cold preservation; Cold + ATL802, transplantation after 4hours of cold preservation with administration of ATL802 during reperfusion; EVLP, transplantation after 4-hours of EVLP; EVLP+ATL802, transplantation after 4-hours of EVLP with administration of ATL802 during ex vivo perfusion. 


\section{Pulmonary Edema}

Compared to Cold, lungs that underwent 4-hours of EVLP without ATL802 had significantly less weight gain from pre-transplantation to post-reperfusion (Cold: $120 \pm 23 \%$ vs. EVLP: $20 \pm 20 \%, p=0.037$ ) and trended towards significance for lungs in EVLP+ATL802 $(28 \pm 10 \%, p=0.056)($ Figure 28A). No significant differences were observed between Cold and Cold+ATL802 (116 $\pm 41 \%)$ or between EVLP and EVLP+ATL802.

\section{Cytokine Expression}

Proinflammatory cytokines were assessed in homogenized tissue supernatant and BAL fluid after the reperfusion period. A significant decrease was identified in levels of IL-12 when comparing EVLP to EVLP+ATL802 (tissue: $132.5 \pm 37.7$ vs. $36.3 \pm 17.5$ pg/mL, p=0.007; BAL: 360.0 \pm 106.7 vs. $85.0 \pm 34.0 \mathrm{pg} / \mathrm{mL}, \mathrm{p}=0.046$ ) (Figure 28B). No other significant differences were identified. 


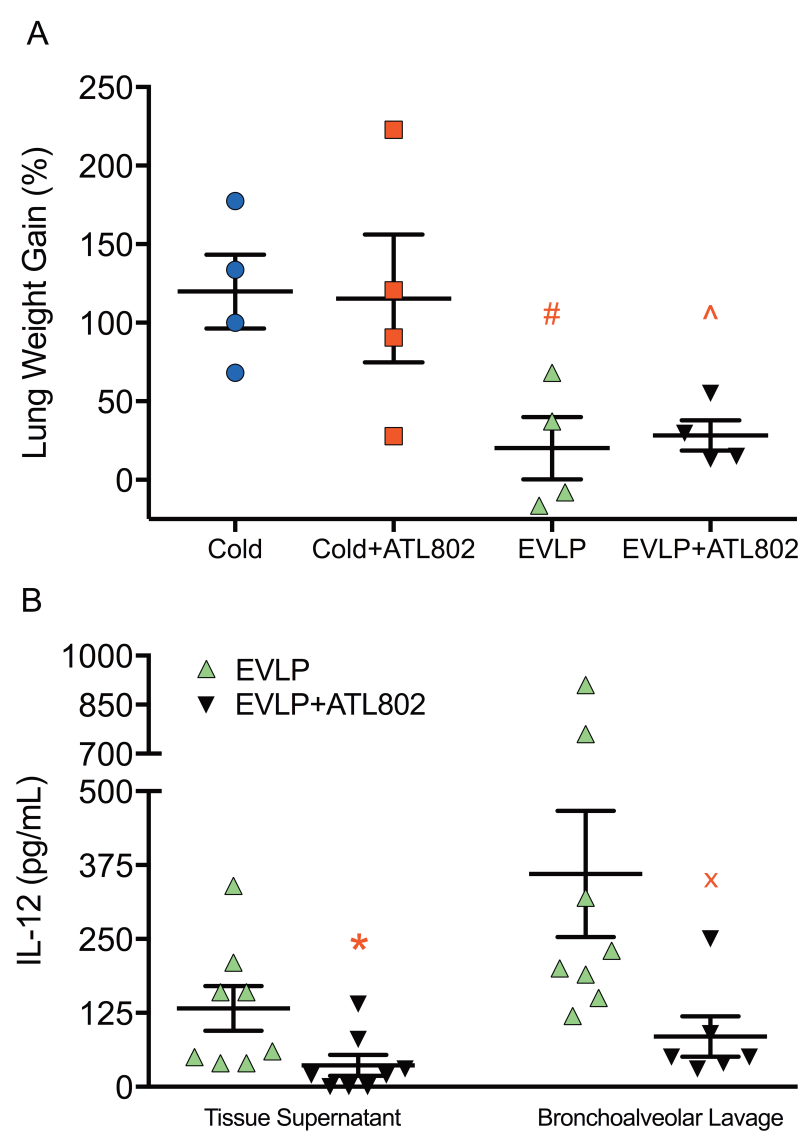

Figure 28: (A) Percentage increase in gross lung weight from pre-transplant to post-reperfusion and (B) tissue supernatant and bronchoalveolar lavage IL-12 levels after reperfusion. Cold, transplantation after 4-hours of cold preservation; Cold + ATL802, transplantation after 4-hours of cold preservation with administration of ATL802 during reperfusion; $E V L P$, transplantation after 4-hours of EVLP; $E V L P+A T L 802$, transplantation after 4-hours of EVLP with administration of ATL802 during ex vivo perfusion. $\# \mathrm{p}=0.037$ vs Cold, ${ }^{\wedge} \mathrm{p}=0.056$ vs Cold, ${ }^{*} \mathrm{p}=0.007, \mathrm{x} \mathrm{p}=0.046$.

\section{Lung Injury Severity Score}

Although EVLP+ATL802 had the lowest mean composite lung injury severity score (comprised of neutrophil infiltration, alveolar edema, and interstitial inflammation), there were no significant differences observed between groups (Cold: 4.31 \pm 0.70 , Cold+ATL802: 3.81 \pm 0.89 , EVLP: 5.94 \pm 0.90, EVLP+ATL802: 2.94 $\pm 1.35, \mathrm{p}=0.24)$. 


\section{Neutrophil Infiltration}

Immunohistochemistry stained slides were compared between all four groups using samples from two distinct lung regions, with five HPF counts per sample. Significantly less neutrophils were seen in group EVLP+ATL802 compared with Cold (56.0 \pm 6.7 vs. $122.8 \pm 16.2$, $\mathrm{p}=0.002)$, and compared with EVLP (56.0 \pm 6.7 vs. $111.7 \pm 10.0, \mathrm{p}=0.012)$ (Figure 29).

A

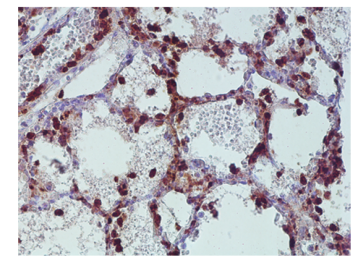

Cold

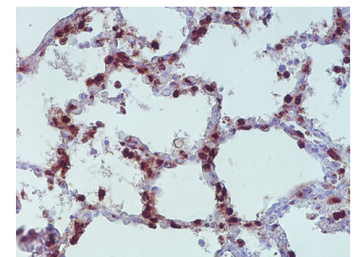

Cold+ATL802

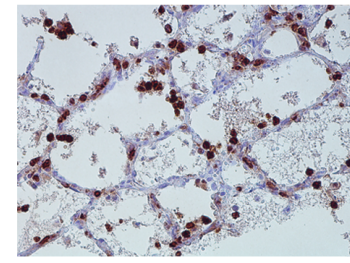

EVLP

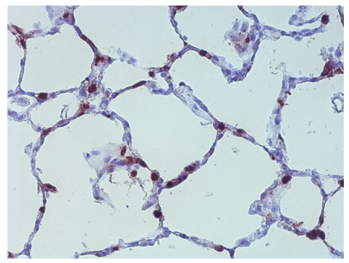

EVLP+ATL802

B

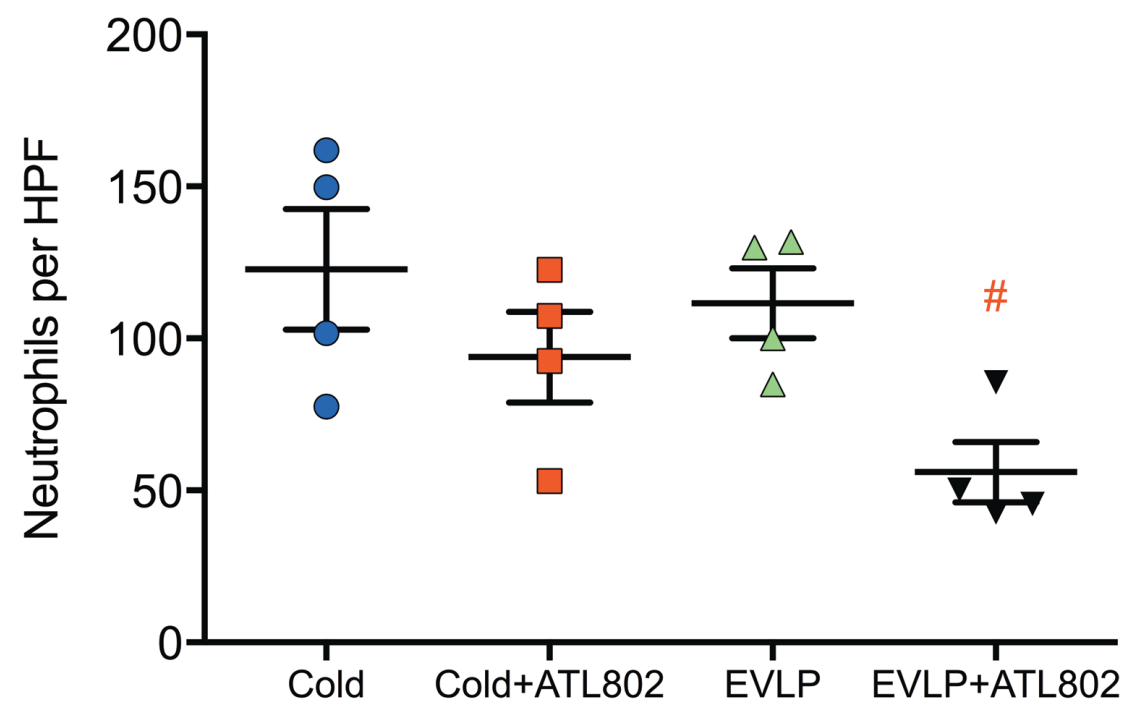

Figure 29: Immunohistochemistry staining for neutrophils. (A) Representative sections (40X magnification). (B) Neutrophils per high-powered field (HPF). Cold, transplantation after 4hours of cold preservation; Cold $+A T L 802$, transplantation after 4-hours of cold preservation with administration of ATL802 during reperfusion; $E V L P$, transplantation after 4-hours of EVLP; EVLP + ATL802, transplantation after 4-hours of EVLP with administration of ATL802 during ex vivo perfusion. $\# \mathrm{p}=0.002$ vs Cold and $\mathrm{p}=0.012$ vs EVLP.

\section{Discussion}

Using a porcine model of left lung transplantation, the present study sought to determine the ability of EVLP, with and without the addition of targeted drug therapy, to rehabilitate DCD 
lungs exposed to 2-hours of warm ischemia. Dynamic compliance 4-hours after reperfusion was improved in lungs treated with 4-hours of EVLP supplemented with an $A_{2 B} R$ antagonist (ATL802), and oxygenation was improved in lungs treated with EVLP alone compared with cold preservation. After the reperfusion period, lungs that received 4-hours of EVLP, with or without ATL802, demonstrated less percent weight gain compared with lungs that underwent 4-hours of cold preservation prior to transplant. The combination of EVLP plus ATL802 significantly reduced neutrophil infiltration after 4-hours of reperfusion, and a decrease in the amount of IL12 expression was observed with the addition of ATL802 compared with EVLP alone. Collectively, DCD lungs exposed to 2-hours of warm ischemia can be transplanted successfully after 4-hours of EVLP as opposed to cold preservation, and targeted drug therapy during the $e x$ vivo perfusion period may further improve outcomes.

Increasing the amount of warm ischemia time acceptable for donor lung procurement would allow for inclusion of uncontrolled DCD lungs from patients who experience cardiac arrest outside of a medical facility, with heparin administration as the only intervention possibly needed before procurement. Further investigation may show that heparin administration is not necessary if lungs are flushed with a fibrinolytic agent prior to EVLP. This strategy for increasing the pool of available donor lungs may shorten wait list times and improve mortality for deteriorating patients with end-stage pulmonary disease, and is gaining support clinically as the supply-demand chasm continues to widen. [102] A case report of successful transplantation after EVLP of human DCD lungs exposed to 4-hours of warm ischemia has been reported. [103] Unfortunately, longer ischemic times increase the risk for IR injury and PGD, but EVLP has been shown to be protective and beneficial when transplanting marginal donor lungs. [104] Our 
results support the use of EVLP compared with cold preservation alone, as the majority of the benefit seen in this study can be attributed to EVLP and not the treatment drug.

The present study employed the use of ATL802, an $\mathrm{A}_{2 \mathrm{~B}} \mathrm{R}$ antagonist, as targeted drug therapy to reduce inflammation and IR injury after transplantation. Both activation and inhibition of $\mathrm{A}_{2 \mathrm{~B}} \mathrm{Rs}$ have been shown to decrease lung inflammation. [48, 97-99] Previous work from our lab implicated resident pulmonary cells as opposed to bone-marrow derived cells as the location of $\mathrm{A}_{2 \mathrm{~B}} \mathrm{R}$ signaling, with a resultant decrease in cytokine expression and neutrophil activation. [97] In a mouse model of acute lung injury, Hogel, S et al. identified alveolar epithelial cells as the location of $\mathrm{A}_{2 \mathrm{~B}} \mathrm{R}$ signaling, which supports the findings from our lab showing attenuation of mouse lung IR with $\mathrm{A}_{2 \mathrm{~B}} \mathrm{R}$ antagonism. $[100,105]$ The present study translated these findings into a large animal transplant model for the first time.

IL-12 is a proinflammatory cytokine secreted by tissue-resident macrophages and dendritic cells, which leads to increased production of interferon- $\gamma$ by T-helper cells. [106] IL12, in conjunction with IL-18, an important component of inflammasome activation, has been implicated in the up-regulation of matrix degrading enzymes and T-cell infiltration in lung injury. [107] Additionally, adenosine signaling has been shown to inhibit IL-12 production, which may further support the use of adenosine receptor-targeted therapies to prevent inflammation. [108] Two IL-12 antagonists, ustekinumab and briakinumab, are currently being tested in clinical trials as targeted drug therapy for immune-mediated inflammatory disease, including graft-vs-host disease. [106] Targeted treatment to down-regulate IL-12 expression may be a possible adjunctive therapy, delivered during the EVLP period, to attenuate IR injury and improve outcomes after lung transplantation. 
Neutrophil activation and infiltration is dependent on various signaling pathways related to both innate and adaptive immunity. We have previously shown that $\mathrm{A}_{2 \mathrm{~A}} \mathrm{R}$ activation significantly attenuates neutrophil infiltration in a mouse model of lung IR injury, as well as after transplantation in a porcine model. [45, 109] Additionally, neutrophil activation has been shown to worsen acute lung injury through the involvement of $A_{3} R s$. [110] The present study shows evidence for a decrease in neutrophil infiltration with ATL802 treatment during EVLP. No significant difference was observed between groups Cold and Cold+ATL802 when ATL802 was administered during reperfusion, as opposed to 4 hours prior to reperfusion (EVLP+ATL802). This timing difference may impact the drug's ability to alter signaling pathways in alveolar epithelial cells and future studies are needed to better understand this effect. The resuscitative requirements of Cold+ATL802 may be due to hemodynamic effects of systemic drug administration.

The findings are limited by low sample size, donor heparin administration, inherent variability with large animals, and the dichotomy between compliance and oxygenation values in EVLP+ATL802. Improved oxygenation was not observed with ATL802 which may be the result of low sample size considering one animal had significantly low oxygenation $\left(\mathrm{PaO}_{2} / \mathrm{FiO}_{2}\right.$ of 84.35). Additionally, post-transplant compliance values represent both lungs. Studies further evaluating the role of $\mathrm{A}_{2 \mathrm{~B}} \mathrm{Rs}$ in acute lung injury are needed prior to making conclusions about the benefit of $\mathrm{A}_{2 \mathrm{~B}} \mathrm{R}$ antagonism as a treatment modality.

The use of EVLP as an assessment and rehabilitation platform to improve organ utilization may lead to a reduction in the critical shortage of donor lungs that meet acceptance criteria. Current functional measurements (oxygenation and compliance) used to assess lung quality may not be sufficient to fully predict success after transplantation, but measuring 
biomarkers, such as endothelin-1 or proinflammatory cytokines, during EVLP may improve our ability to correctly predict outcomes. [111, 112] Additionally, EVLP provides an opportunity to provided targeted rehabilitative strategies based on the needs of each individual set of donor lungs. The administration of inhaled $\beta 2$-agonist and neutrophil elastase during EVLP has been shown to improve outcomes after transplantation. $[113,114]$ Potentially more beneficial is the opportunity to provide drug treatments, such as antibiotics, at concentration levels that would otherwise be detrimental if systemically administered to the recipient. [115]

In summary, the use of EVLP to rehabilitate uncontrolled DCD lungs subjected to prolonged warm ischemia is promising. Our findings of improved outcomes after transplantation with the use of EVLP and targeted drug therapy during the ex vivo perfusion period suggest that it may be possible to successfully transplant lungs procured from patients suffering cardiac death in the field. Expanding current acceptance criteria for donor lung procurement is needed to decrease wait list times and improve wait list mortality for patients with end-stage pulmonary disease. Using EVLP to assess lung quality and administer targeted drug therapies prior to recipient allocation will allow for maximal organ utilization and may improve outcomes after transplantation. 


\section{PROJECT \#5}

\section{TRPV4 Channels Mediate Pulmonary Inflammation \\ During Ischemia-Reperfusion Injury}

Eric J. Charles MD ${ }^{1}$, Yunge Zhao MD PhD ${ }^{1}$, Kwanseok Hong ${ }^{2}$, Corina Marziano ${ }^{2}$, J. Hunter Mehaffey $\mathrm{MD}^{2}$, Irving L. Kron $\mathrm{MD}^{1}$, Swapnil K. Sonkusare $\mathrm{PhD}^{2}$, Victor E. Laubach $\mathrm{PhD}^{1}$, Ashish K Sharma MBBS $\mathrm{PhD}^{1}$

Departments of ${ }^{1}$ Surgery and ${ }^{2}$ Molecular Physiology and Biological Physics University of Virginia, Charlottesville, Virginia

\section{Status of Research: ONGOING EXPERIMENTS}

Summary of data to date are presented below. 


\section{Introduction}

Transient receptor potential vanilloid-4 (TRPV4) cation channels have a preference for calcium, are activated by various inflammatory stimuli, and are thought to be involved in the pathophysiology of acute lung injury. [116-119] In a mouse model of ventilator-induced lung injury, TRPV4 channels were found to contribute to acute vascular permeability by allowing rapid calcium influx. [118] Membrane stretch, mechanical stimuli, heat, and epoxyeicosatrienoic acids, along with other products of inflammation activate TRPV4 channels. [118] TRPV4 channels are fairly ubiquitous, and are expressed in a wide variety of tissues and organs, including the lung and vascular endothelium. $[118,120]$ In this study, we demonstrate a role for TPRV4 channels in the regulation of endothelial permeability and epithelial activation during lung IRI.

\section{Materials and Methods}

Our murine model of left lung hilar occlusion to induce IRI was used with C57BL6 wildtype (WT) mice and TRPV4 $4^{-/}$mice. Mice underwent sham surgery or 1 hour of left lung ischemia, followed by 2 hours of reperfusion. A TRPV4 specific antagonist, GSK2193874 $(1 \mathrm{mg} / \mathrm{kg}$, administered intravenously 1 hour before ischemia) was used in separate groups of WT mice. Lung function was measured as previously described (compliance, airway resistance, and pulmonary artery pressure using an isolated, buffer-perfused ventilation apparatus).

Bronchoaveolar lavage fluid was obtained at the conclusion of the reperfusion period and used to measure cytokine and myeloperoxidase levels. Lung edema was assessed by wet/dry weight and neutrophil infiltration was quantified with immunohistochemistry. Murine alveolar type II epithelial cells (MLE12) and lung primary microvascular endothelial cells (PMVEC) were 
evaluated in vitro using a model of hypoxia/reoxygenation (HR; 3 hours/1 hour) or TNF- $\alpha$ $(50 \mu \mathrm{M})$ administration to assess the role of TRPV4 channels by quantifying CXCL1 production, calcium influx, and neutrophil transmigration.

\section{Results}

Deletion of TRPV channels in our mouse model attenuates lung IRI as demonstrated by increased pulmonary compliance ( $5.1 \pm 0.3$ vs. $\left.2.5 \pm 0.1 \mu \mathrm{l} / \mathrm{cm} \mathrm{H}_{2} \mathrm{O} ; \mathrm{p}<0.01\right)$, decreased airway resistance (1.6 \pm 0.1 vs. $2.1 \pm 0.2 \mathrm{~cm} \mathrm{H}_{2} \mathrm{O} / \mu \mathrm{l} / \mathrm{sec} ; \mathrm{p}=0.03$ ), and decreased pulmonary artery pressure (7.7 \pm 0.2 vs. $12.8 \pm 0.4 \mathrm{~cm} \mathrm{H}_{2} \mathrm{O} ; \mathrm{p}<0.01$, Figure 30A). Likewise, treatment of WT mice with GSK2193874 attenuates lung IR to a similar degree as TRPV4 channel deletion (Figure 30B).

Neutrophil infiltration and myeloperoxidase production were significantly reduced in $\mathrm{TRPV}^{-/}$mice (Figure 31). Proinflammatory cytokine production (CXCL1, IL-17, TNF- $\alpha$, and HMGB1) was also significantly attenuated in $\mathrm{TRPV}^{-/-}$mice (Figure 32). In vitro analyses of the effect of TRPV4 channels on MLE12 cells and PMVECs demonstrated that TRPV4 channels are involved in CXCL1 production from MLE12 cells and calcium influx and neutrophil transmigration in PMVECs and that TRPV4 antagonism attenuates epithelial activation and endothelial permeability (Figure 33). 

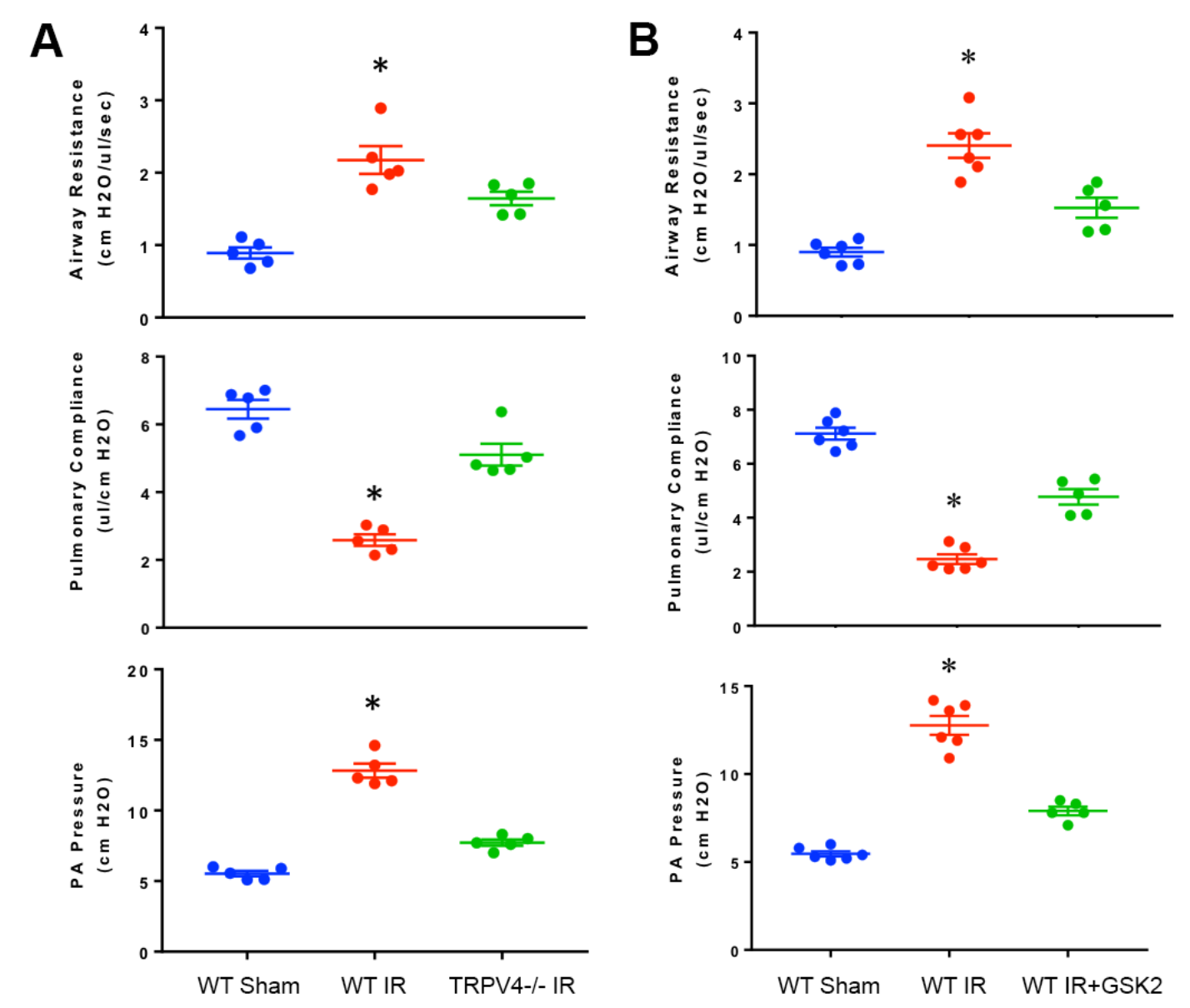

Figure 30: Pulmonary function results (airway resistance, pulmonary compliance, and pulmonary artery pressure) demonstrating attenuation of lung IRI with (A) TRPV4 ${ }^{-/}$IR mice and (B) mice treated with GSK2193874 (WT IR+GSK2) compared with wild-type (WT) sham and WT IR mice. ${ }^{*} \mathrm{p}<0.05$ vs. other groups.
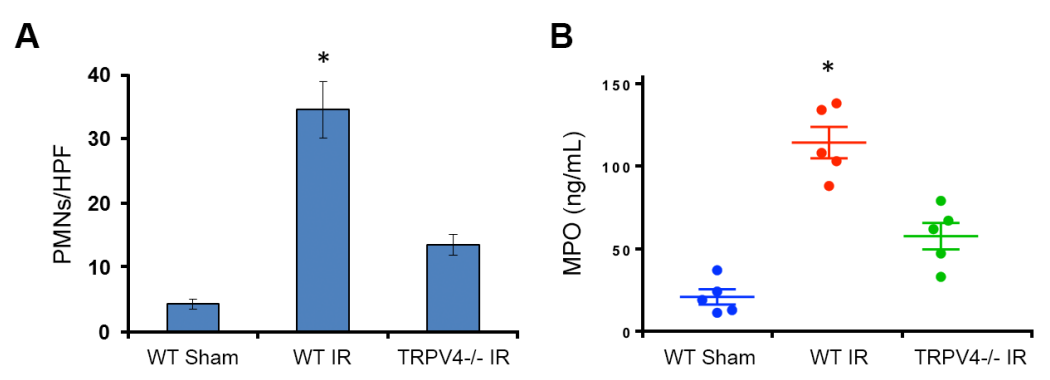

Figure 31: (A) Neutrophil infiltration (neutrophils per histologic high-powered field, PMNs/HPF) and (B) myeloperoxidase production were significantly reduced in TRPV4 ${ }^{-/}$IR mice compared with wild-type sham (WT sham) and wild-type IR mice (WT IR). ${ }^{*} \mathrm{p}<0.05$ vs. other groups. 

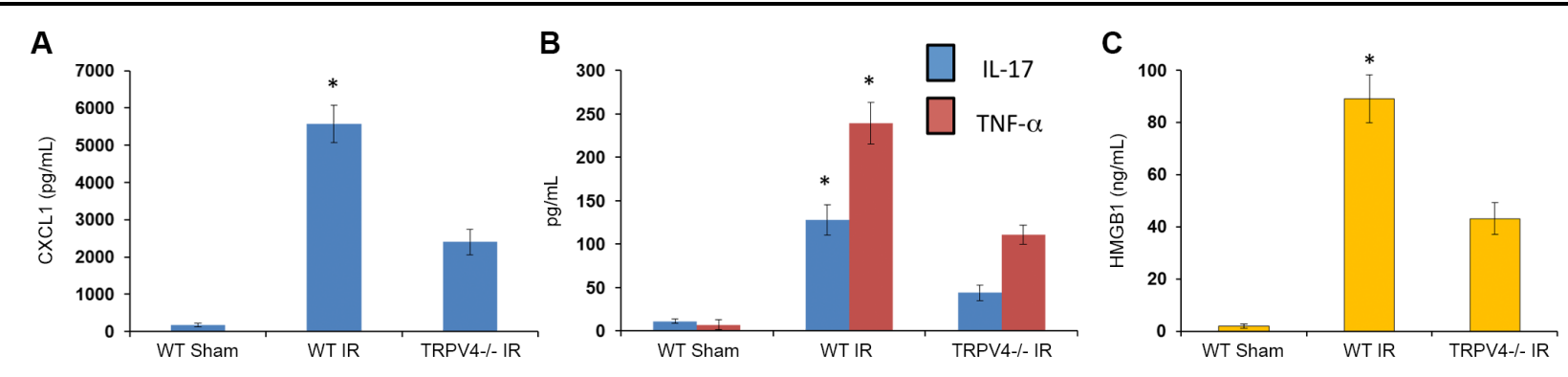

Figure 32: Proinflammatory cytokine production: (A) CXCL1, (B) IL-17 and TNF- $\alpha$, and (C) HMGB1 was significantly attenuated in TRPV4 ${ }^{-/-}$IR mice compared with wild-type IR mice (WT IR). ${ }^{*} \mathrm{p}<0.05$ vs. other groups.
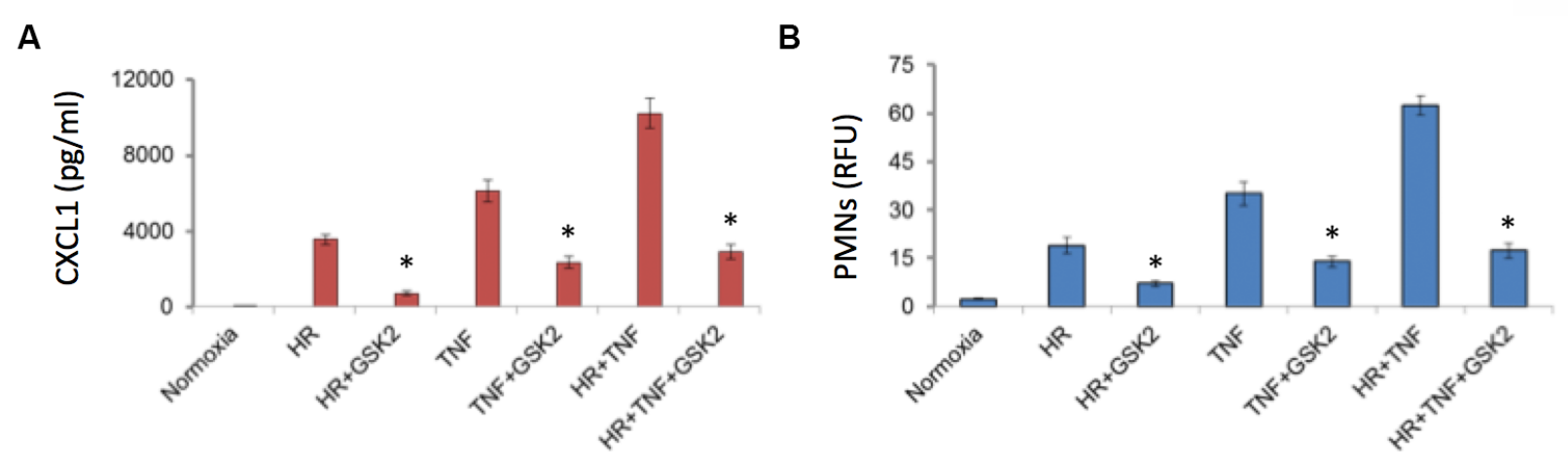

Figure 33: TRPV4 antagonism with GSK2193874 (GSK2) attenuates (A) murine alveolar type II epithelial cell activation as demonstrated by reduced CXCL1 production and (B) primary microvascular endothelial cell permeability as demonstrated by reduced neutrophil transmigration. $H R, 3$ hours hypoxia and 1 hour reoxygenation; $T N F$, stimulation with TNF- $\alpha$ $(50 \mu \mathrm{M}) ; R F U$, relative fluorescence unit. ${ }^{*} \mathrm{p}<0.05$ vs. corresponding group without GSK2.

\section{Conclusions}

TRPV4 cation channels are involved in lung IRI, mediating neutrophil infiltration, proinflammatory cytokine production, and pulmonary dysfunction. TRPV4 antagonism is a potential pharmacologic target to attenuate lung IRI injury. Further experiments are needed to better understand the role of calcium influx via TRPV4 channels and the regulation of vascular integrity and epithelial cell function. 


\title{
PROJECT \#6
}

\section{Pannexin-1 Channels on Endothelial Cells Mediate Vascular Inflammation During Lung Ischemia-Reperfusion Injury}

\author{
Ashish K Sharma MBBS $\mathrm{PhD}^{1}$, Eric J. Charles $\mathrm{MD}^{1}$, Yunge Zhao MD $\mathrm{PhD}^{1}$, \\ Adishesh K. Narahari ${ }^{2}$, Pranav K. Baderdinni ${ }^{2}$, Miranda E. Good PhD ${ }^{3}$, Irving L. Kron MD ${ }^{1}$, \\ Douglas A. Bayliss $\mathrm{PhD}^{2}$, Kodi S. Ravichandran $\mathrm{PhD}^{4}$, Brant E. Isakson $\mathrm{PhD}^{3}$, \\ Victor E. Laubach $\mathrm{PhD}^{1}$
}

Departments of ${ }^{1}$ Surgery, ${ }^{2}$ Pharmacology, ${ }^{3}$ Molecular Physiology and Biological Physics, and

${ }^{4}$ Microbiology, Immunology, and Cancer Biology University of Virginia, Charlottesville, Virginia

\section{Status of Research: MANUSCRIPT CURRENTLY UNDER REVIEW}

My Role: I performed animal surgeries and assisted in generating data for this publication. Summary and data excerpts presented below, along with additional hypotheses that I tested but were not included in the publication. 


\section{Introduction}

Panx1 is a ubiquitously expressed hemichannel that is permeable to many different metabolites, including ATP. [121, 122] After lung IRI, extracellular ATP is one of many different DAMPs that act as a pro-inflammatory signal leading to the recruitment of circulating neutrophils that further perpetuate the inflammatory cascade. [123, 124] Purinergic P2 receptors bind extracellular ATP. There are ATP-gated cation channels, P2X 1-7, and G-protein coupled receptors, $\mathrm{P} 2 \mathrm{Y}$ 1,2,4,6,11-14. P2X receptors are specific for ATP while P2Y receptors can bind ATP, ADP, UDP, and UTP. [125] During lung IRI, the specific mechanisms by which ATP is released and propagates the inflammatory pathways leading to lung injury are not well known. Of the three pannexin channels (Panx 1-3), Panx1 is the most well understood and is known to release intracellular ATP. [121, 126] Additionally, endothelial cell Panx1 has been shown to open in response to TNF- $\alpha$ during acute inflammation and be involved in leukocyte adhesion and emigration through endothelium. [127] Building on these data, we hypothesized that Panx1 plays an important role in purinergic signaling during lung IRI and that the release of extracellular ATP via endothelial cell Panx1 channels is an important signaling mechanism that results in vascular permeability and leukocyte migration into lung tissue (Figure 34). Our results demonstrate that endothelial Panx1 is a major contributor to vascular permeability, pulmonary edema accumulation, inflammation, leukocyte infiltration, and physiologic dysfunction during IRI. The remaining aspects of the Panx1 signaling mechanism in lung IRI are still being explored with ongoing experiments. 


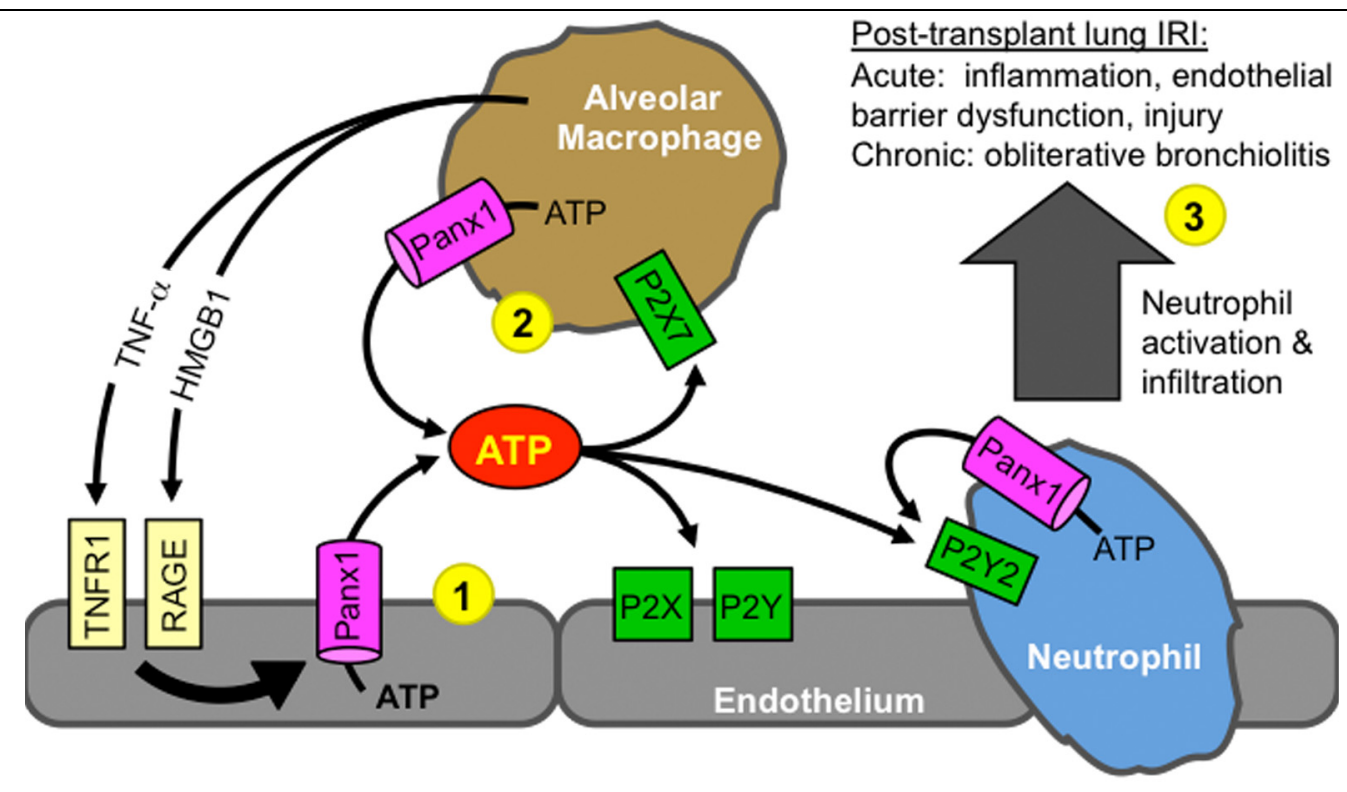

Figure 34: Schematic of proposed Panx1-purinergic signaling pathways involved in lung IRI. (1) ATP release from endothelial cells via Panx1 mediates vascular permeability and leukocyte emigration into tissue. (2) Alveolar macrophages secrete TNF- $\alpha$, which is known to stimulate Panx1 activation on endothelial cells, along with HMGB1. ATP release by macrophages leads to autocrine or paracrine signaling via $\mathrm{P} 2 \mathrm{X}$ and $\mathrm{P} 2 \mathrm{Y}$ receptors. (3) Autocrine Panx1-ATP signaling via P2Y2 on neutrophils leads to neutrophil activation and infiltration, endothelial barrier disruption, vascular permeability, and pulmonary dysfunction.

\section{Methods and Results}

Using our mouse model of left lung hilar clamp to induce IRI, carbenoxolone (CBX, 0.25 $\mathrm{mg} / \mathrm{kg}$ ) and probenecid (PBN, $1.1 \mathrm{mg} / \mathrm{kg})$, two well-established Panx1 inhibitors, were tested to determine the role of Panx1 signaling during acute lung inflammation. [128] Intraperitoneal injections were administered 1 hour prior to ischemia, after which the mice underwent 1 hour of ischemia followed by 2 hours of reperfusion. Both CBX and PBN attenuated lung IRI (as measured by airway resistance, pulmonary compliance, and pulmonary artery pressure) when compared with untreated wild type C57BL6 mice (Figure 35). 
A

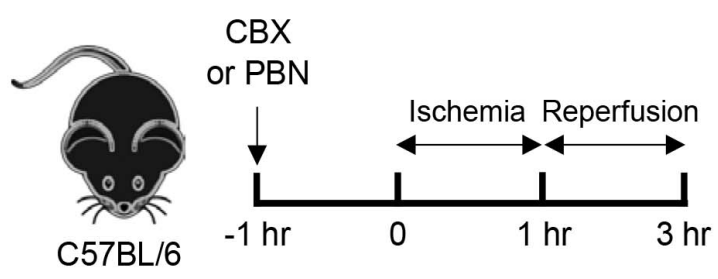

C

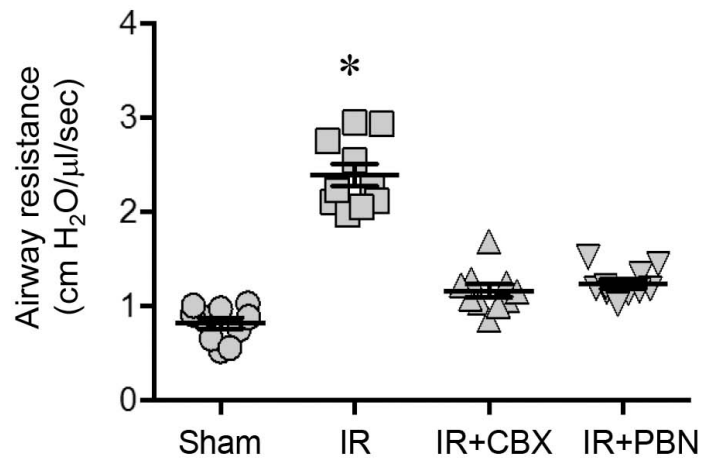

B

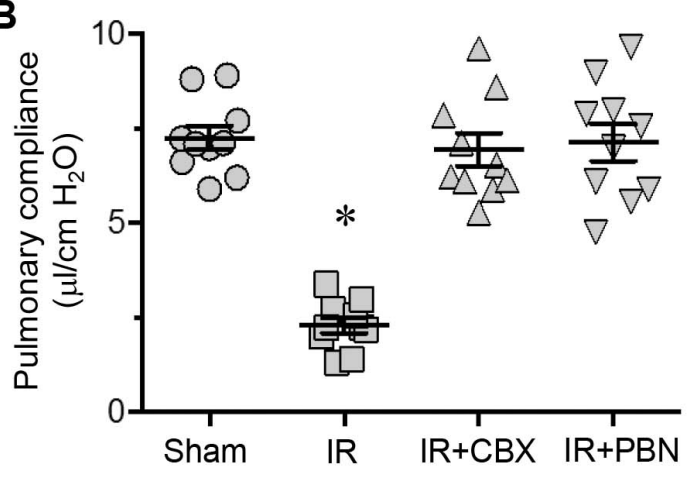

D

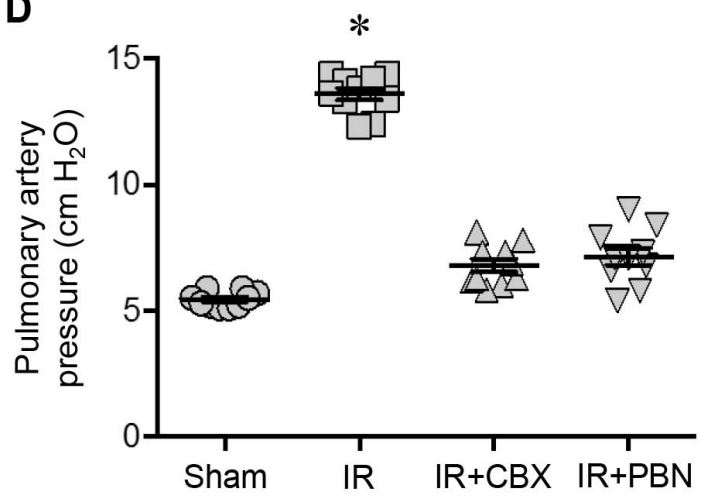

Figure 35: Pulmonary function results. (A) Schematic of mouse hilar clamp IR model. IRI causes severe pulmonary dysfunction: decreased compliance (B), increased airway resistance (C), and increased pulmonary artery pressure (D). Pannexin 1 inhibition with carbenoxolone $(\mathrm{CBX})$ or probenecid (PBN) attenuates lung IRI and improves all function parameters. $\mathrm{n}=10 /$ group, $* \mathrm{p}<0.05$ vs. all other groups.

Panx1 inhibition with CBX and PBN also attenuated lung edema (Figure 36A and B) and pro-inflammatory cytokine (TNF- $\alpha$, IL-17, CXCL1) production (Figure 36C and D). 
A

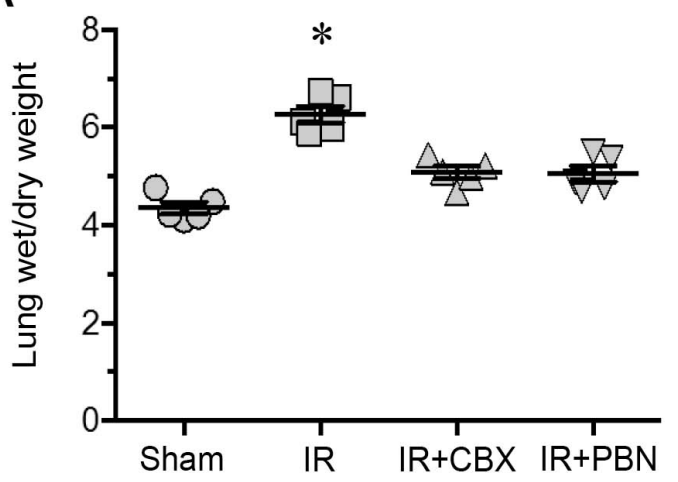

C

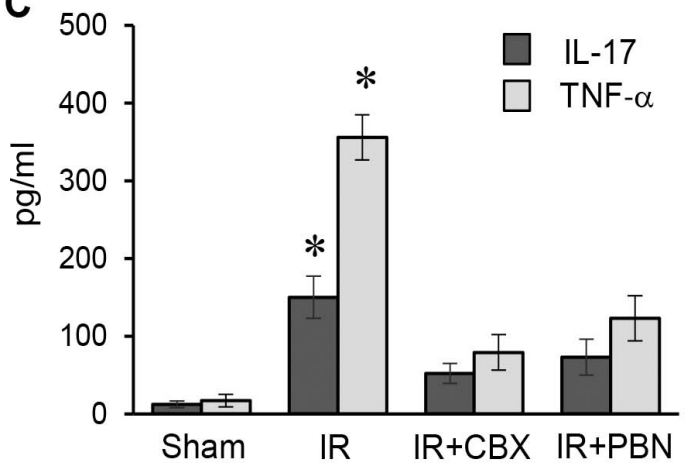

B

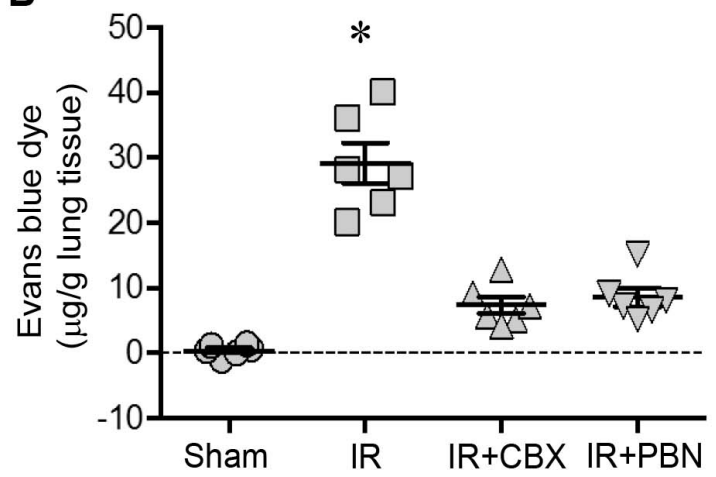

D

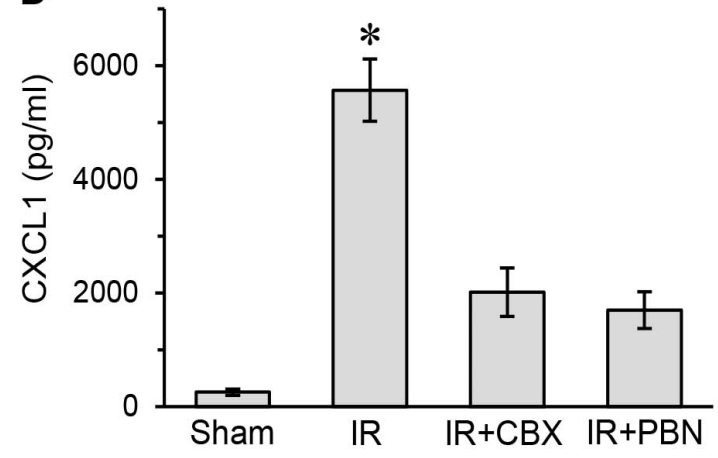

Figure 36: (A) Lung wet/dry weight and (B) Evans blue dye extravasation (two indicators of pulmonary edema) were elevated after IRI, but significantly reduced in animals pretreated with carbenoxolone (CBX) or probenecid (PBN). (C, D) Proinflammatory cytokines TNF- $\alpha$, IL-17, and CXCL1 were significantly attenuated in animals pretreated with CBX or PBN. $n=5-8 /$ group, ${ }^{*} \mathrm{p}<0.05$ vs. all other groups.

To investigate the role of endothelial cell specific Panx1, tamoxifen-induced VE-Cad Panx ${ }^{f / f l}$ mice (provided by Dr. Brant Isakson) were used in our mouse model of acute lung IRI. Compared with VE-Cad Panx $1^{\mathrm{fl} / \mathrm{fl}}$ mice treated with vehicle only, tamoxifen-treated mice demonstrated attenuation of lung dysfunction (compliance, airway resistance, pulmonary artery pressure, Figure 37). 

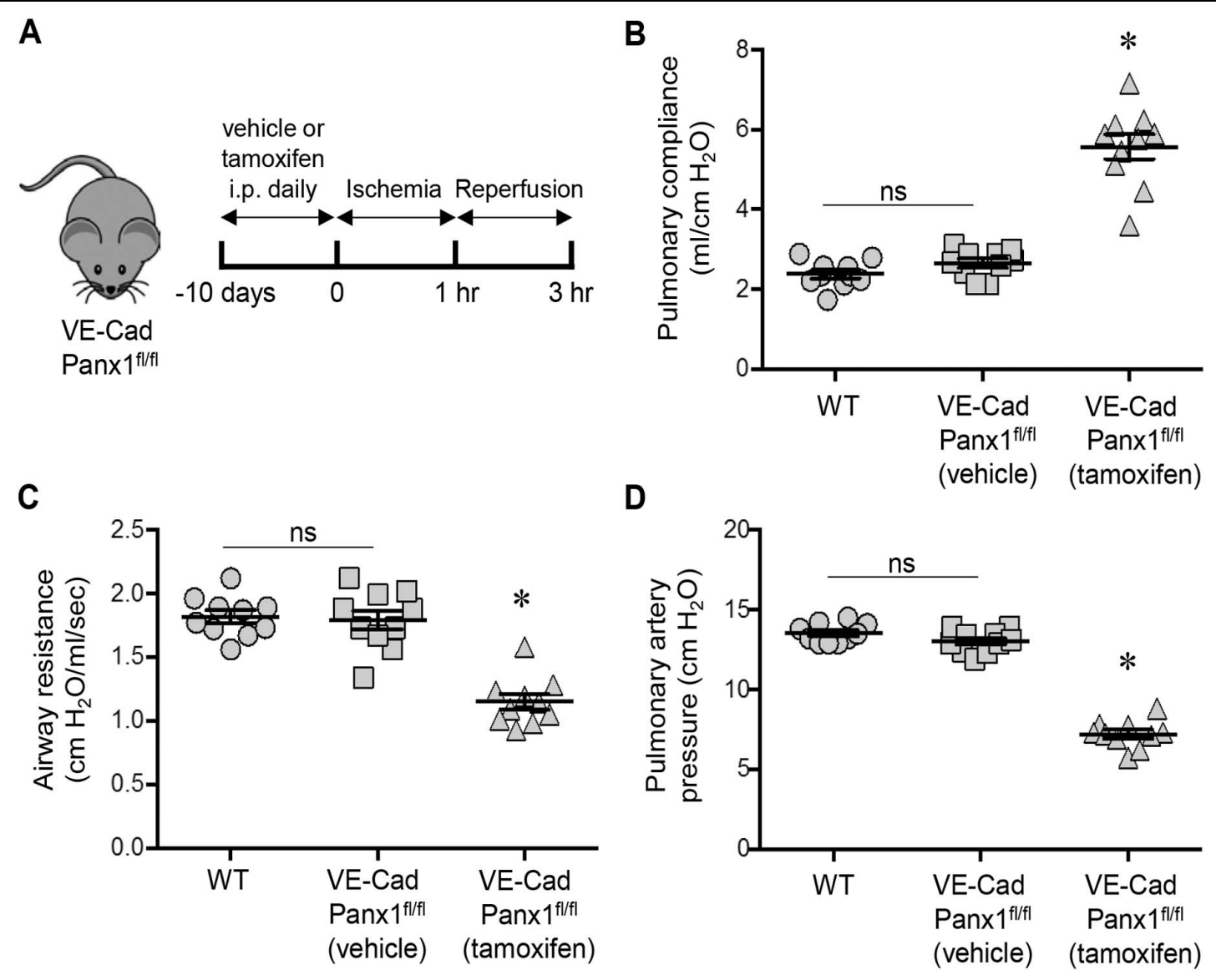

Figure 37: Pulmonary function results. (A) Schematic of mouse hilar clamp IR model with VECad Panx $1^{\mathrm{fl} / \mathrm{fl}}$ mice treated for 10 days with either vehicle or tamoxifen. IRI causes severe pulmonary dysfunction: decreased compliance $(B)$, increased airway resistance $(C)$, and increased pulmonary artery pressure (D) in vehicle-treated VE-Cad Panx $1^{\mathrm{fl} / \mathrm{fl}}$ mice. Tamoxifentreated VE-Cad Panx $1^{\mathrm{fl} / \mathrm{fl}}$ mice demonstrated significant attenuation of lung injury. $\mathrm{n}=10$ /group, ${ }^{*} \mathrm{p}<0.05$ vs VE-Cad Panx $1^{\mathrm{fl} / \mathrm{fl}}$ mice (vehicle); $n s$, not significant.

Tamoxifen-treated VE-Cad Panx $1^{\mathrm{fl} / \mathrm{fl}}$ mice also demonstrated reduced lung edema and lower levels of proinflammatory cytokines (TNF- $\alpha$, IL-17, CXCL1, Figure 38). 

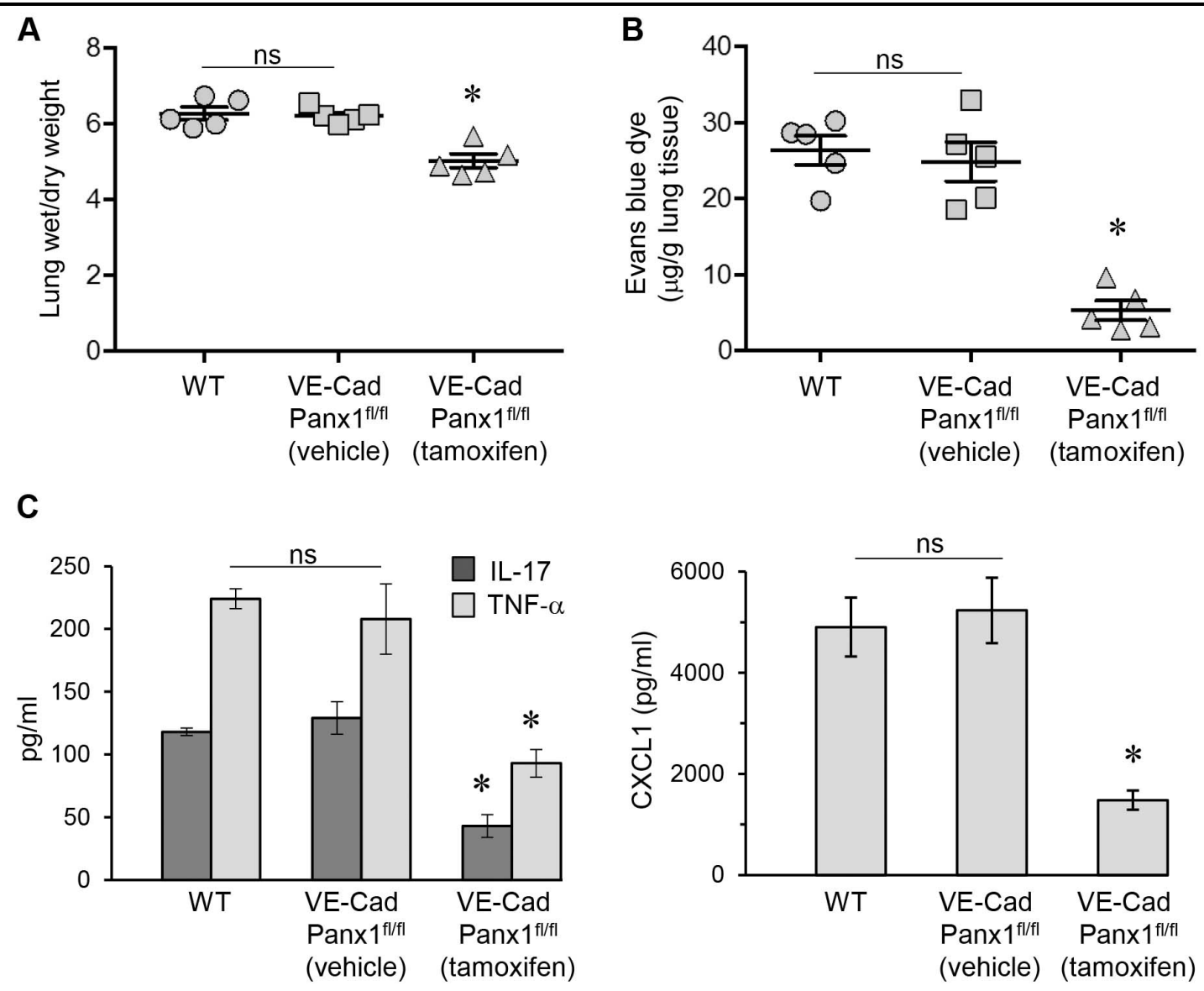

Figure 38: (A) Lung wet/dry weight and (B) Evans blue dye extravasation (two indicators of pulmonary edema) were elevated in wild type (WT) and vehicle-treated VE-Cad Panx $1^{\mathrm{fl} / \mathrm{fl}}$ mice, but significantly reduced in tamoxifen-treated VE-Cad Panx $1^{\mathrm{fl} / \mathrm{fl}}$ mice. (C, D) Proinflammatory cytokines TNF- $\alpha$, IL-17, and CXCL1 were significantly attenuated in tamoxifen-treated VE-Cad Pan $\times 1^{\mathrm{fl} / \mathrm{fl}}$ mice. $\mathrm{n}=5-7 /$ group, ${ }^{*} \mathrm{p}<0.05$ vs. all other groups; $n s$, not significant.

Building on our results that endothelial cell specific Panx 1 mediates vascular permeability, edema accumulation, and proinflammatory cytokine production, I sought to determine a potential role for Panx 1 on bone marrow-derived immune cells (versus non-immune cells) in lung IRI. To test this, I performed a bone marrow transplant experiment using wild-type C57BL6 mice and Panx $1^{-/ /}$donor and recipient mice. Following 6 weeks of recovery, lung function was measured after sham thoracotomy or 1 hour of ischemia followed by 2 hours of reperfusion (IR). No significant differences were observed between groups that underwent IR 
(Figure 39). Although we believe that Panx 1 channels mediate acute lung inflammation, Panx $1^{-/-}$ mice may have compensatory upregulation of Panx2 or Panx3, allowing for continued purinergic signaling. This may explain the absence of protection from IRI observed in irradiated Panx $1^{-/-}$ mice that received Panx $1^{-/-}$bone marrow. The role of $\operatorname{Panx} 1^{-/-}$on bone-marrow-derived immune cells is not apparent from these preliminary experiments and will be further investigated.

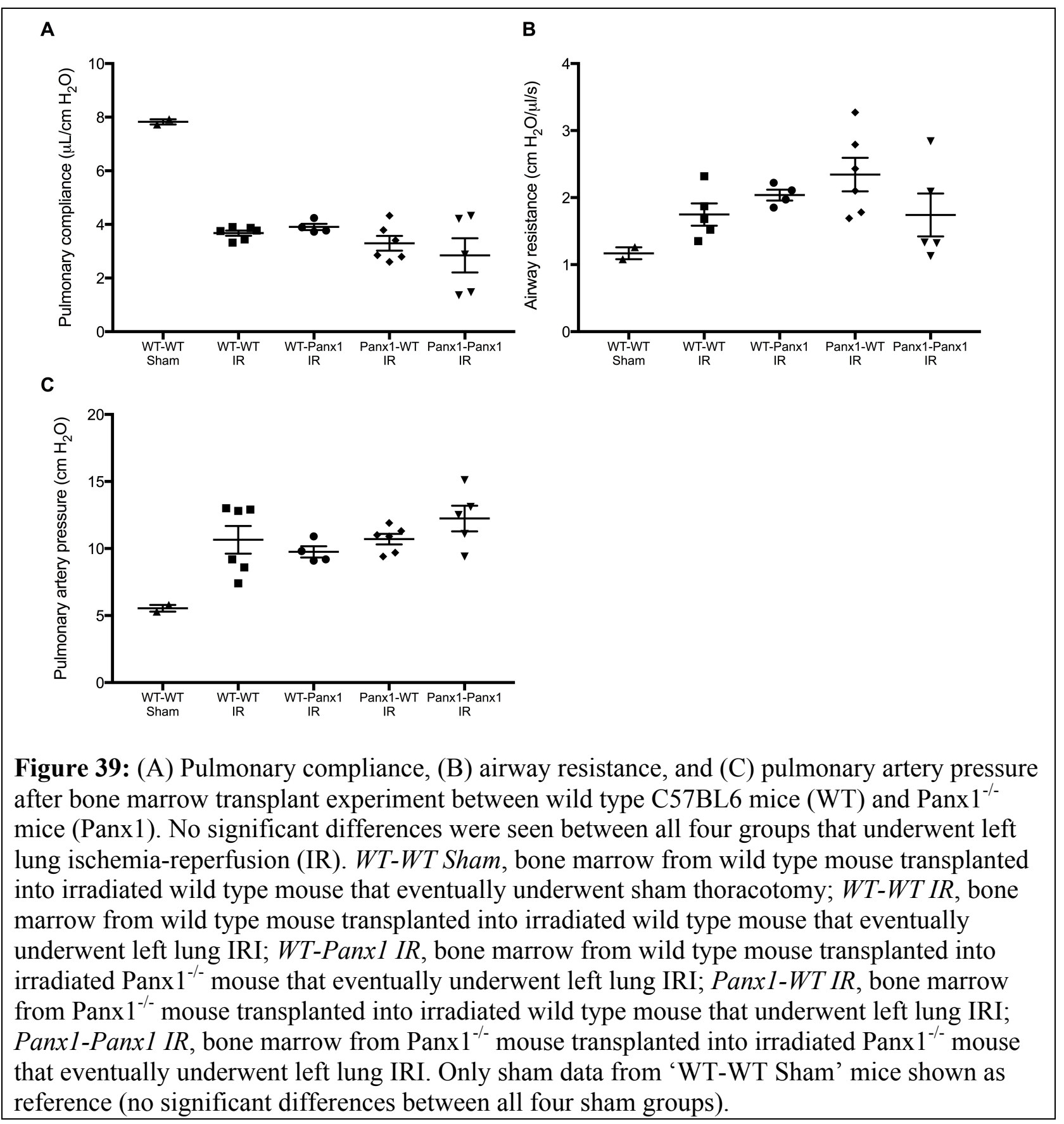


Additional experiments were performed to investigate the role of $\mathrm{P} 2 \mathrm{X} 7$ and $\mathrm{P} 2 \mathrm{Y} 2$ receptors in lung IRI using $\mathrm{P} 2 \mathrm{X} 7^{-/-}$and $\mathrm{P} 2 \mathrm{Y} 2^{-/-}$mice. Results were similar to the Panx $1^{-/-}$bone marrow chimeras, with no significant protection observed in either strain of purinergic receptor knockout mice (Figure 40). Signaling through purinergic receptors other than P2X7 and P2Y2 may account for the lack of any differences observed on lung function assessment. The role of P2X7 and P2Y2 receptors is not apparent in our mouse model of lung IRI and will be further investigated. These preliminary results will need to be confirmed by repeating the current experiments and by performing new experiments using specific P2X7 and P2Y2 pharmacologic inhibitors.

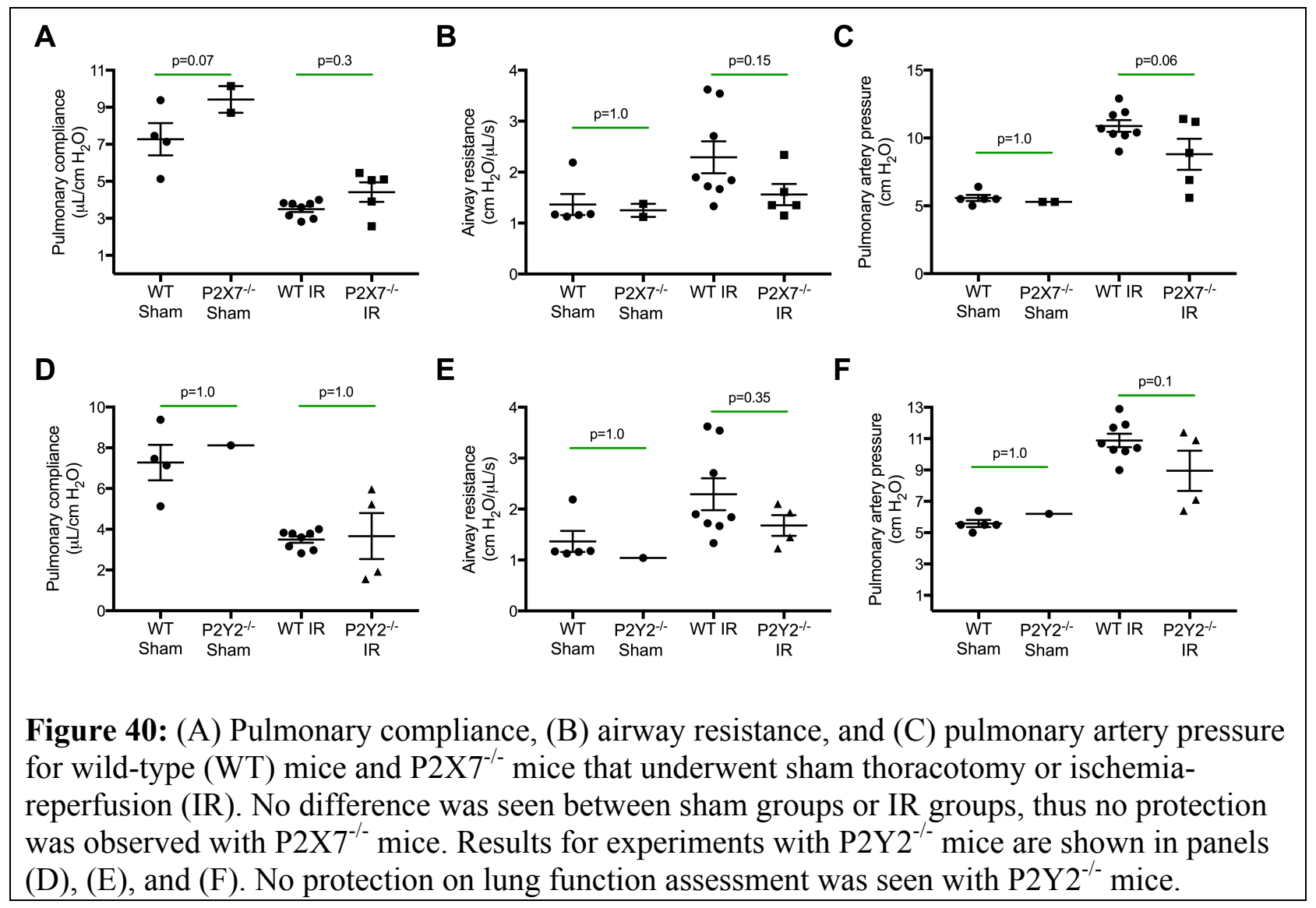




\section{Conclusions}

Endothelial Panx1 channels are important contributors to lung IRI mainly through increased vascular permeability leading to edema accumulation and pulmonary dysfunction. The use of Panx1 inhibitors that block the Panx1-ATP purinergic pathway is a promising therapy for attenuation of lung IRI. Ongoing experiments will continue to explore the role of Panx1 on alveolar macrophages, epithelial cells, and neutrophils, as well as the interaction between Panx1mediated ATP release and purinergic $\mathrm{P} 2 \mathrm{X}$ and $\mathrm{P} 2 \mathrm{Y}$ receptors during lung IRI. Our preliminary results regarding the role of Panx 1 on bone marrow-derived immune cells and the role of P2X7 and P2Y2 receptors during lung IR will be further investigated. Our mouse model of left lung IRI may not be sufficient to identify differences between wild type, $\mathrm{Panx} 1^{-/-}, \mathrm{P} 2 \mathrm{X} 7^{-/-}$, and $\mathrm{P} 2 \mathrm{Y} 2^{-/-}$ mice and thus future experiments will focus on the in vitro activation (ATP release, proinflammatory cytokine production, cell transmigration) of specific cell types (alveolar macrophages, alveolar epithelial cells, and neutrophils) in the presence or absence of Panx1, P2X7, and P2Y2 pharmacologic inhibitors. 


\section{Conclusions}

IRI is the major driving force behind poor clinical outcomes after lung transplantation. There are currently no good ways to predict, diagnose, or treat lung IRI, and when it progresses to PGD, it is associated with significant increases in morbidity and mortality rates. Without improvements in prevention, diagnosis, and treatment, lung IRI will continue to plague transplant clinicians and patients alike, limiting the possibility that patients with end-stage pulmonary disease will become the recipients of successful lung transplants.

The work presented here sought to address the effect of IRI on lung transplant outcomes through three main avenues: 1) the use of EVLP as an assessment and reconditioning platform for marginal donor lungs, 2) the development of an in vivo leukocyte-labeling molecular probe with SPECT imaging for early, noninvasive diagnosis and monitoring of IRI, and 3) the investigation of various molecular targets as potential pharmacological treatment options. Improvement of the diagnosis and management of lung IRI will lead to improved clinical outcomes after lung transplantation and an increase in the number of patients on the waiting list that receive a lung transplant.

My first focus of study determined that EVLP can improve the utilization of marginal donor lungs, specifically lungs procured after circulatory death. Our results question currently established limitations on acceptable cold and warm ischemic times and argue for extending the guidelines to include more sets of marginal donor lungs. Additionally, these findings suggest that organ allocation and distribution can be improved without compromising allograft function using a strategy that combines EVLP reconditioning with cold preservation. Next, I explored the effect of increasing warm ischemia time on allograft function, as warm ischemia is thought to 
alter lung immune cell function and lead to cellular death. Our results found that longer warm ischemia times may be acceptable for lung transplantation if reconditioned first with EVLP.

As acceptance criteria for marginal donor lungs are expanded, there will inevitably be an increase in the incidence of IRI. Therefore, my second focus of study was to develop an in vivo leukocyte-labeling molecular probe that can be used with SPECT as a new diagnostic technique that would allow noninvasive imaging of lung IRI. By targeting FPR1 with ${ }^{99 \mathrm{~m}}$ Tc-cFLFLF, we demonstrated that active inflammation can be specifically imaged using SPECT, and that resolution of injury over time can be monitored using this technique. As pharmacologic interventions are developed, having a noninvasive method to identify specific immune cell populations involved in each patient's acute lung injury and then monitor the effect of treatments may improve therapeutic success rates.

My third focus of study was to discover new mechanisms of lung IRI and to identify novel molecular targets for the therapeutic prevention or treatment of IRI. Donor A2BRs were found to play an important role in lung IRI and that pretreatment of the donor organ with ATL802, an A2BR antagonist, while on EVLP can attenuate post-transplant IRI and improve functional outcomes. Additionally, TRPV4 cation channels that control cellular calcium influx were identified to be important contributors to lung IRI and are a promising therapeutic target to reduce endothelial permeability and epithelial activation using GSK2193874, a TRPV4 specific antagonist. Finally, endothelial cell Pannexin 1 channels were identified as important mediators of lung IRI through the release of ATP, a DAMP molecule, and that targeted inhibition of Panx1 with carbenoxolone or probenecid reduces the release of proinflammatory cytokines TNF- $\alpha$, IL17, and CXCL1, as well as reduces vascular permeability and edema accumulation. 


\section{Future Directions}

Lung Allograft Assessment with EVLP

Incorporating EVLP into lung transplant programs has opened the door for potential utilization of marginal donor organs that were previously considered unacceptable for transplantation. Our research findings suggest that EVLP preserves and reconditions marginal donor lungs allowing them to withstand post-EVLP cold ischemia. The combination of normothermic ex vivo perfusion with subsequent cold preservation may be favorable for cellular metabolism and survival but further investigations are needed to fully understand this relationship. Future research will focus on the limitations of such reconditioning, as the amount of ischemic time prior to and after EVLP will dictate how organs can be allocated and distributed. The idea of organ assessment and repair centers is promising and has been proposed, but an improved understanding of the benefits of normothermic perfusion versus cold preservation is needed. [50, 129]

Organ ischemic time contributes to the risk of IRI, limiting the enthusiasm for transplanting marginal organs. However, our results suggest that substantially more warm ischemic time than is currently accepted can be tolerated with the use of EVLP. Additional studies are needed that expand upon our finding that up to 120 minutes of warm ischemia prior to EVLP is not an absolute contraindication to procuring, assessing, and potentially transplanting DCD lungs. The cellular effects of warm ischemia may be mitigated with normothermic perfusion during EVLP but further investigation is needed. The incorporation of uncontrolled DCD organs into transplant procurement protocols would be a significant contribution to the supply of available organs. 


\section{Molecular Imaging of Lung IRI}

The limiting factor of expanded use of marginal donor lungs is the inability to diagnose and effectively treat IRI. The interaction between resident alveolar macrophages, iNKT-cells, vascular endothelium, alveolar epithelial cells, and circulating polymorphonuclear leukocytes complicates our ability to accurately diagnose and to rapidly and appropriately treat IRI on a cellular level. While our research findings are promising regarding the use of cFLFLF for in vivo leukocyte labeling with SPECT imaging, cFLFLF largely targets activated neutrophils (and perhaps some macrophages), which are an important contributor to lung IRI but not the sole celltype involved. Additionally, it is known that activation of resident iNKT-cells and alveolar macrophages occurs earlier than neutrophil infiltration. $[25,26,28]$ The next steps to expand upon our current findings will focus on targeting M1 macrophages or activated iNKT-cells to provide an even earlier diagnosis of the onset of IRI (long before PGD occurs). Additionally, for resolution of lung IRI (either endogenous resolution or resolution in response to therapy) to be effectively monitored, we are planning future experiments that will investigate the use of molecular imaging probes that target M2 macrophages (anti-inflammatory macrophages that are thought to be involved in the resolution of inflammation). A targeted approach to the multiphasic inflammatory process of lung IRI will improve diagnostic capabilities. New receptor targets should be explored such as macrophage mannose receptor (on M2 macrophages) and folate receptors (on M1 macrophages). Additionally, it is unlikely that any one specific imaging probe will be sufficient for all patients with different stages of IRI, therefore the combination of multiple different cell-type specific imaging probes into one drug cocktail should be explored. As such, a great advantage of SPECT imaging is the capability to simultaneously detect and differentiate the uptake of different probes labeled with different isotopes (e.g. ${ }^{99 \mathrm{~m}} \mathrm{Tc},{ }^{111} \mathrm{In}$, or 
${ }^{64} \mathrm{Cu}$ ). PET imaging will likely play a significant role in future research in this area as it can provide improved resolution and shorter image acquisition time. Thus, another important future direction will involve the comparison of SPECT versus PET imaging of lung IRI using cellspecific molecular probes.

\section{Molecular Targets to Attenuate Lung IR Injury}

In my opinion, ex vivo rehabilitation of organs is the future of transplantation. Our finding that supplementing EVLP with an A2BR antagonist can improve outcomes after transplantation alludes to the fact that multiple different treatments can be given to recondition damaged organs while on ex vivo perfusion circuits without the inherent side effects of systemically treating the donor or the recipient. EVLP research should focus on specific cellular modulation, gene therapy, and problem-specific targeted treatments that can be given ex vivo and will contribute to reduction of IRI.

Molecular targets that allow for safe, effective pharmacologic treatment of IRI are needed. Patients with IRI that progresses to PGD after lung transplantation have worse outcomes compared with patients who do not have evidence of IRI. TRPV4 channels are shown to be involved in epithelial activation and endothelial permeability in acute lung injury via alteration of calcium homeostasis. Pharmacologic antagonism of TRPV4 channel activation may be a successful preventative therapy or treatment for lung IRI but additional research is needed in both small animal as well as clinically relevant large animal transplant studies.

Panx1 channels appear to be important mediators of inflammation as well. While our research has identified a role for endothelial Panx1 in IRI, we do not yet understand the contribution of Panx1 (or purinergic receptors that bind ATP) on macrophages, epithelial cells, 
and other involved cell types. The signaling pathways by which Panx1 activation contributes to leukocyte activation and infiltration during IRI is unknown, but likely involves ATP release. Activation of downstream purinergic receptors (via autocrine or paracrine signaling of ATP) contributing to a positive feedback loop is possible and will require further investigation. The translation of these small animal and in vitro experiments to a large animal, preclinical transplant model is a logical first step to validate these findings and will be necessary for better prediction of the clinical applicability. 


\section{Acknowledgements}

As a second year general surgery resident with extremely limited prior research experience, I was welcomed into a high performing lung transplant research laboratory and given the education, equipment, experiences and mentorship necessary to excel. For that I am forever grateful and indebted to Dr. Victor Laubach and Dr. Irving Kron. They have taught me the true meaning of being a scientist. My success in the laboratory would not have been possible without the tireless support of Dr. Ashish Sharma, who shared his vision for projects, his technical skills and knowledge, and his dedication to research with me. To Dr. Avril Somlyo, Dr. Brant Isakson, Dr. Mark Okusa, and Dr. Gilbert Upchurch, thank you for taking time out of your busy schedules to meet with me and share your guidance and knowledge. The many hours I spent in the laboratory would have been a lot less productive without the constant presence and support I received from Mr. Tony Herring, Dr. Yunge Zhao, Ms. Cindy Dodson, and Ms. Sheila Hammond. My co-residents who shared the highs and lows of research with me for the past few years are an amazing group of intelligent, hard working, inquisitive physicians who pushed me each and every day. They will go on to accomplish great feats in medicine and research and I am honored to have shared this time and these experiences with them. To my wife, my parents, and my siblings, thank you for the unending support and encouragement. You have inspired and encouraged me every step of the way and have reminded me to work hard at making a difference. I hope this work will contribute to the betterment of patient outcomes and for that it will all be worth it. 


\section{References}

1. Yusen RD, Edwards LB, Kucheryavaya AY, Benden C, Dipchand AI, Goldfarb SB, et al. The Registry of the International Society for Heart and Lung Transplantation: Thirtysecond Official Adult Lung and Heart-Lung Transplantation Report--2015; Focus Theme: Early Graft Failure. J Heart Lung Transplant. 2015;34:1264-77.

2. U.S. Department of Health \& Human Services. Organ Procurement and Transplantation Network: National Data. Available at: https:// https://optn.transplant.hrsa.gov/data/viewdata-reports/nationaldata/. Accessed May 1, 2017.

3. Valapour M, Skeans MA, Heubner BM, Smith JM, Hertz MI, Edwards LB, et al. OPTN/SRTR 2013 Annual Data Report: lung. Am J Transplant. 2015;15 Suppl 2:1-28.

4. Valapour M, Skeans MA, Smith JM, Edwards LB, Cherikh WS, Callahan ER, et al. Lung. Am J Transplant. 2016;16 Suppl 2:141-68.

5. Rana A, Gruessner A, Agopian VG, Khalpey Z, Riaz IB, Kaplan B, et al. Survival benefit of solid-organ transplant in the United States. JAMA Surg. 2015;150:252-9.

6. Cypel M, Keshavjee S. The clinical potential of ex vivo lung perfusion. Expert Rev Respir Med. 2012;6:27-35.

7. Barnieh L, Baxter D, Boiteau P, Manns B, Doig C. Benchmarking performance in organ donation programs: dependence on demographics and mortality rates. Can J Anaesth. 2006;53:727-31.

8. van Suylen V, Luijk B, Hoek RAS, van de Graaf EA, Verschuuren EA, Van De Wauwer $\mathrm{C}$, et al. A multicenter study on long-term outcomes after lung transplantation comparing donation after circulatory death and donation after brain death. Am J Transplant. 2017.

9. Krutsinger D, Reed RM, Blevins A, Puri V, De Oliveira NC, Zych B, et al. Lung transplantation from donation after cardiocirculatory death: a systematic review and meta-analysis. J Heart Lung Transplant. 2015;34:675-84.

10. Cypel M, Levvey B, Van Raemdonck D, Erasmus M, Dark J, Love R, et al. International Society for Heart and Lung Transplantation Donation After Circulatory Death Registry Report. J Heart Lung Transplant. 2015;34:1278-82.

11. Wigfield C. Donation after cardiac death for lung transplantation: a review of current clinical practice. Curr Opin Organ Transplant. 2014;19:455-9.

12. Mason DP, Thuita L, Alster JM, Murthy SC, Budev MM, Mehta AC, et al. Should lung transplantation be performed using donation after cardiac death? The United States experience. J Thorac Cardiovasc Surg. 2008;136:1061-6.

13. Arcasoy SM, Fisher A, Hachem RR, Scavuzzo M, Ware LB, Dysfunction IWGoPLG. Report of the ISHLT Working Group on Primary Lung Graft Dysfunction part V: predictors and outcomes. J Heart Lung Transplant. 2005;24:1483-8.

14. Christie JD, Edwards LB, Kucheryavaya AY, Aurora P, Dobbels F, Kirk R, et al. The Registry of the International Society for Heart and Lung Transplantation: twenty-seventh official adult lung and heart-lung transplant report--2010. J Heart Lung Transplant. 2010;29:1104-18.

15. Christie JD, Kotloff RM, Ahya VN, Tino G, Pochettino A, Gaughan C, et al. The effect of primary graft dysfunction on survival after lung transplantation. Am J Respir Crit Care Med. 2005;171:1312-6. 
16. Daud SA, Yusen RD, Meyers BF, Chakinala MM, Walter MJ, Aloush AA, et al. Impact of immediate primary lung allograft dysfunction on bronchiolitis obliterans syndrome. Am J Respir Crit Care Med. 2007;175:507-13.

17. Cypel M, Keshavjee S. Extending the donor pool: rehabilitation of poor organs. Thorac Surg Clin. 2015;25:27-33.

18. Palestro CJ. In vivo leukocyte labeling: the quest continues. J Nucl Med. 2007;48:332-4.

19. Chaney J, Suzuki Y, Cantu E, 3rd, van Berkel V. Lung donor selection criteria. J Thorac Dis. 2014;6:1032-8.

20. de Perrot M, Young K, Imai Y, Liu M, Waddell TK, Fischer S, et al. Recipient T cells mediate reperfusion injury after lung transplantation in the rat. $J$ Immunol. 2003;171:4995-5002.

21. Ross SD, Tribble CG, Gaughen JR, Jr., Shockey KS, Parrino PE, Kron IL. Reduced neutrophil infiltration protects against lung reperfusion injury after transplantation. Ann Thorac Surg. 1999;67:1428-33; discussion 34.

22. Sharma AK, LaPar DJ, Zhao Y, Li L, Lau CL, Kron IL, et al. Natural killer T cellderived IL-17 mediates lung ischemia-reperfusion injury. Am J Respir Crit Care Med. 2011;183:1539-49.

23. Stammberger U, Gaspert A, Hillinger S, Vogt P, Odermatt B, Weder W, et al. Apoptosis induced by ischemia and reperfusion in experimental lung transplantation. Ann Thorac Surg. 2000;69:1532-6.

24. Yang Z, Sharma AK, Marshall M, Kron IL, Laubach VE. NADPH oxidase in bone marrow-derived cells mediates pulmonary ischemia-reperfusion injury. Am J Respir Cell Mol Biol. 2009;40:375-81.

25. Zhao M, Fernandez LG, Doctor A, Sharma AK, Zarbock A, Tribble CG, et al. Alveolar macrophage activation is a key initiation signal for acute lung ischemia-reperfusion injury. Am J Physiol Lung Cell Mol Physiol. 2006;291:L1018-26.

26. den Hengst WA, Gielis JF, Lin JY, Van Schil PE, De Windt LJ, Moens AL. Lung ischemia-reperfusion injury: a molecular and clinical view on a complex pathophysiological process. Am J Physiol Heart Circ Physiol. 2010;299:H1283-99.

27. Al-Mehdi AB, Zhao G, Dodia C, Tozawa K, Costa K, Muzykantov V, et al. Endothelial NADPH oxidase as the source of oxidants in lungs exposed to ischemia or high $\mathrm{K}+$. Circ Res. 1998;83:730-7.

28. de Perrot M, Liu M, Waddell TK, Keshavjee S. Ischemia-reperfusion-induced lung injury. Am J Respir Crit Care Med. 2003;167:490-511.

29. Fisher AB, Dodia C, Tan ZT, Ayene I, Eckenhoff RG. Oxygen-dependent lipid peroxidation during lung ischemia. J Clin Invest. 1991;88:674-9.

30. Ishiyama T, Dharmarajan S, Hayama M, Moriya H, Grapperhaus K, Patterson GA. Inhibition of nuclear factor kappaB by IkappaB superrepressor gene transfer ameliorates ischemia-reperfusion injury after experimental lung transplantation. J Thorac Cardiovasc Surg. 2005;130:194-201.

31. Kaminski A, Pohl CB, Sponholz C, Ma N, Stamm C, Vollmar B, et al. Up-regulation of endothelial nitric oxide synthase inhibits pulmonary leukocyte migration following lung ischemia-reperfusion in mice. Am J Pathol. 2004;164:2241-9.

32. Zhao G, al-Mehdi AB, Fisher AB. Anoxia-reoxygenation versus ischemia in isolated rat lungs. Am J Physiol. 1997;273:L1112-7. 
33. Moore TM, Khimenko P, Adkins WK, Miyasaka M, Taylor AE. Adhesion molecules contribute to ischemia and reperfusion-induced injury in the isolated rat lung. $J$ Appl Physiol (1985). 1995;78:2245-52.

34. Christie JD, Sager JS, Kimmel SE, Ahya VN, Gaughan C, Blumenthal NP, et al. Impact of primary graft failure on outcomes following lung transplantation. Chest. 2005;127:161-5.

35. Van Raemdonck D, Neyrinck A, Cypel M, Keshavjee S. Ex-vivo lung perfusion. Transpl Int. 2015;28:643-56.

36. Kondo T, Chen F, Ohsumi A, Hijiya K, Motoyama H, Sowa T, et al. beta-Adrenoreceptor Agonist Inhalation During Ex Vivo Lung Perfusion Attenuates Lung Injury. Ann Thorac Surg. 2015.

37. Cypel M, Liu M, Rubacha M, Yeung JC, Hirayama S, Anraku M, et al. Functional repair of human donor lungs by IL-10 gene therapy. Sci Transl Med. 2009;1:4ra9.

38. Mulloy DP, Stone ML, Crosby IK, Lapar DJ, Sharma AK, Webb DV, et al. Ex vivo rehabilitation of non-heart-beating donor lungs in preclinical porcine model: delayed perfusion results in superior lung function. J Thorac Cardiovasc Surg. 2012;144:120815.

39. Cypel M, Yeung JC, Liu M, Anraku M, Chen F, Karolak W, et al. Normothermic ex vivo lung perfusion in clinical lung transplantation. N Engl J Med. 2011;364:1431-40.

40. Wagner CE, Pope NH, Charles EJ, Huerter ME, Sharma AK, Salmon MD, et al. Ex vivo lung perfusion with adenosine A2A receptor agonist allows prolonged cold preservation of lungs donated after cardiac death. J Thorac Cardiovasc Surg. 2015.

41. Stone ML, Sharma AK, Zhao Y, Charles EJ, Huerter ME, Johnston WF, et al. Sphingosine-1-phosphate receptor 1 agonism attenuates lung ischemia-reperfusion injury. Am J Physiol Lung Cell Mol Physiol. 2015;308:L1245-52.

42. Algahim MF, Love RB. Donation after circulatory death: the current state and technical approaches to organ procurement. Curr Opin Organ Transplant. 2015;20:127-32.

43. Sharing UNfO. 2013 Transplant Data. 2013.

44. US Department of Health and Human Services Organ Procurement and Transplantation Network.

45. LaPar DJ, Laubach VE, Emaminia A, Crosby IK, Hajzus VA, Sharma AK, et al. Pretreatment strategy with adenosine A2A receptor agonist attenuates reperfusion injury in a preclinical porcine lung transplantation model. J Thorac Cardiovasc Surg. 2011;142:887-94.

46. Emaminia A, Lapar DJ, Zhao Y, Steidle JF, Harris DA, Laubach VE, et al. Adenosine A(2)A agonist improves lung function during ex vivo lung perfusion. Ann Thorac Surg. 2011;92:1840-6.

47. Cypel M, Yeung JC, Hirayama S, Rubacha M, Fischer S, Anraku M, et al. Technique for prolonged normothermic ex vivo lung perfusion. J Heart Lung Transplant. 2008;27:1319-25.

48. Zhao Y, LaPar DJ, Steidle J, Emaminia A, Kron IL, Ailawadi G, et al. Adenosine signaling via the adenosine $2 \mathrm{~B}$ receptor is involved in bronchiolitis obliterans development. J Heart Lung Transplant. 2010;29:1405-14.

49. Wigfield CH, Cypel M, Yeung J, Waddell T, Alex C, Johnson C, et al. Successful emergent lung transplantation after remote ex vivo perfusion optimization and transportation of donor lungs. Am J Transplant. 2012;12:2838-44. 
50. Black SM WB. Regional Organ Assessment and Repair Centers (ARCs). IJMBS. 2013;5:243-46.

51. Yang D, Guo S, Zhang T, Li H. Hypothermia attenuates ischemia/reperfusion-induced endothelial cell apoptosis via alterations in apoptotic pathways and JNK signaling. FEBS Lett. 2009;583:2500-6.

52. Hsin MK, Iskender I, Nakajima D, Chen M, Kim H, Dos Santos PR, et al. Extension of donor lung preservation with hypothermic storage after normothermic ex vivo lung perfusion. J Heart Lung Transplant. 2016;35:130-6.

53. Steinbrook R. Organ donation after cardiac death. N Engl J Med. 2007;357:209-13.

54. Kootstra G, Daemen JH, Oomen AP. Categories of non-heart-beating donors. Transplant Proc. 1995;27:2893-4.

55. Mehaffey JH, Charles EJ, Sharma AK, Money DT, Zhao Y, Stoler MH, et al. Airway pressure release ventilation during ex vivo lung perfusion attenuates injury. $J$ Thorac Cardiovasc Surg. 2017;153:197-204.

56. Charles EJ, Mehaffey JH, Sharma AK, Zhao Y, Stoler MH, Isbell JM, et al. Lungs donated after circulatory death and prolonged warm ischemia are transplanted successfully after enhanced ex vivo lung perfusion using adenosine A2B receptor antagonism. J Thorac Cardiovasc Surg. 2017.

57. Wagner CE, Pope NH, Charles EJ, Huerter ME, Sharma AK, Salmon MD, et al. Ex vivo lung perfusion with adenosine A2A receptor agonist allows prolonged cold preservation of lungs donated after cardiac death. J Thorac Cardiovasc Surg. 2016;151:538-45.

58. Charles EJ, Huerter ME, Wagner CE, Sharma AK, Zhao Y, Stoler MH, et al. Donation After Circulatory Death Lungs Transplantable Up to Six Hours After Ex Vivo Lung Perfusion. Ann Thorac Surg. 2016;102:1845-53.

59. Machuca TN, Cypel M. Ex vivo lung perfusion. J Thorac Dis. 2014;6:1054-62.

60. Wagner CE, Pope NH, Charles EJ, Huerter ME, Sharma AK, Salmon MD, et al. Ex vivo lung perfusion with adenosine A2A receptor agonist allows prolonged cold preservation of lungs donated after cardiac death. J Thorac Cardiovasc Surg. 2016;151:538-46.

61. Levvey BJ, Harkess M, Hopkins P, Chambers D, Merry C, Glanville AR, et al. Excellent clinical outcomes from a national donation-after-determination-of-cardiac-death lung transplant collaborative. Am J Transplant. 2012;12:2406-13.

62. Gries CJ, White DB, Truog RD, Dubois J, Cosio CC, Dhanani S, et al. An official American Thoracic Society/International Society for Heart and Lung Transplantation/Society of Critical Care Medicine/Association of Organ and Procurement Organizations/United Network of Organ Sharing Statement: ethical and policy considerations in organ donation after circulatory determination of death. Am J Respir Crit Care Med. 2013;188:103-9.

63. Gamez P, Cordoba M, Ussetti P, Carreno MC, Alfageme F, Madrigal L, et al. Lung transplantation from out-of-hospital non-heart-beating lung donors. one-year experience and results. J Heart Lung Transplant. 2005;24:1098-102.

64. Nunez JR, Varela A, del Rio F, Gamez P, Calatayud J, Cordoba M, et al. Bipulmonary transplants with lungs obtained from two non-heart-beating donors who died out of hospital. J Thorac Cardiovasc Surg. 2004;127:297-9.

65. Miyoshi K, Oto T, Otani S, Tanaka S, Harada M, Kakishita T, et al. Effect of donor premortem hypoxia and hypotension on graft function and start of warm ischemia in 
donation after cardiac death lung transplantation. J Heart Lung Transplant. 2011;30:44551.

66. Nijkamp DM, van der Bij W, Verschuuren EA, Heemskerk MB, de Buijzer E, Erasmus ME. Non-heart-beating lung donation: how big is the pool? J Heart Lung Transplant. 2008;27:1040-2.

67. Brown CR, Shafii AE, Farver CF, Murthy SC, Pettersson GB, Mason DP. Pathologic correlates of heparin-free donation after cardiac death in lung transplantation. $J$ Thorac Cardiovasc Surg. 2013;145:e49-50.

68. Erasmus ME, Verschuuren EA, Nijkamp DM, Vermeyden JW, van der Bij W. Lung transplantation from nonheparinized category III non-heart-beating donors. A singlecentre report. Transplantation. 2010;89:452-7.

69. Martens A, Boada M, Vanaudenaerde BM, Verleden SE, Vos R, Verleden GM, et al. Steroids can reduce warm ischemic reperfusion injury in a porcine donation after circulatory death model with ex vivo lung perfusion evaluation. Transpl Int. 2016;29:1237-46.

70. Nakajima D, Chen F, Okita K, Motoyama H, Hijiya K, Ohsumi A, et al. Reconditioning lungs donated after cardiac death using short-term hypothermic machine perfusion. Transplantation. 2012;94:999-1004.

71. Nakajima D, Chen F, Yamada T, Sakamoto J, Ohsumi A, Bando T, et al. Reconditioning of lungs donated after circulatory death with normothermic ex vivo lung perfusion. $J$ Heart Lung Transplant. 2012;31:187-93.

72. Hardy JD. The first lung transplant in man (1963) and the first heart transplant in man (1964). Transplant Proc. 1999;31:25-9.

73. Diamond JM, Lee JC, Kawut SM, Shah RJ, Localio AR, Bellamy SL, et al. Clinical risk factors for primary graft dysfunction after lung transplantation. Am J Respir Crit Care Med. 2013;187:527-34.

74. Locke LW, Chordia MD, Zhang Y, Kundu B, Kennedy D, Landseadel J, et al. A novel neutrophil-specific PET imaging agent: cFLFLFK-PEG-64Cu. J Nucl Med. 2009;50:7907.

75. Zhang Y, Xiao L, Chordia MD, Locke LW, Williams MB, Berr SS, et al. Neutrophil targeting heterobivalent SPECT imaging probe: cFLFLF-PEG-TKPPR-99mTc. Bioconjug Chem. 2010;21:1788-93.

76. Le Y, Murphy PM, Wang JM. Formyl-peptide receptors revisited. Trends Immunol. 2002;23:541-8.

77. Xiao L, Zhang Y, Liu Z, Yang M, Pu L, Pan D. Synthesis of the Cyanine 7 labeled neutrophil-specific agents for noninvasive near infrared fluorescence imaging. Bioorg Med Chem Lett. 2010;20:3515-7.

78. Yang Z, Sharma AK, Linden J, Kron IL, Laubach VE. CD4+ T lymphocytes mediate acute pulmonary ischemia-reperfusion injury. J Thorac Cardiovasc Surg. 2009;137:695702; discussion 02.

79. Stolin A, Wojcik R, Williams M. Conference Record of the IEEE Nuclear Science Symposium and Medical Imaging Conference, San Diego, CA. 2006.

80. Zhao Y-G, Xiao A-Z, Newcomer RG, Park HI, Kang T, Chung LWK, et al. Activation of pro-gelatinase $\mathrm{B}$ by endometase/matrilysin-2 promotes invasion of human prostate cancer cells. Journal of Biological Chemistry. 2003;278:15056-64. 
81. Zhao Y-G, Xiao A-Z, Park HI, Newcomer RG, Yan M, Man Y-G, et al. Endometase/matrilysin-2 in human breast ductal carcinoma in situ and its inhibition by tissue inhibitors of metalloproteinases-2 and -4: a putative role in the initiation of breast cancer invasion. Cancer Research. 2004;64:590-8.

82. Orfila C, Lepert JC, Gossart S, Frisach MF, Cambon C, Pipy B. Immunocytochemical characterization of lung macrophage surface phenotypes and expression of cytokines in acute experimental silicosis in mice. Histochem J. 1998;30:857-67.

83. Wenceslau CF, Szasz T, McCarthy CG, Baban B, NeSmith E, Webb RC. Mitochondrial $\mathrm{N}$-formyl peptides cause airway contraction and lung neutrophil infiltration via formyl peptide receptor activation. Pulm Pharmacol Ther. 2016;37:49-56.

84. Mandal P, Novotny M, Hamilton TA. Lipopolysaccharide induces formyl peptide receptor 1 gene expression in macrophages and neutrophils via transcriptional and posttranscriptional mechanisms. J Immunol. 2005; 175:6085-91.

85. Yang X, Chordia MD, Du X, Graves JL, Zhang Y, Park YS, et al. Targeting formyl peptide receptor 1 of activated macrophages to monitor inflammation of experimental osteoarthritis in rat. J Orthop Res. 2016;34:1529-38.

86. Stasiuk GJ, Smith H, Wylezinska-Arridge M, Tremoleda JL, Trigg W, Luthra SK, et al. Gd3 + cFLFLFK conjugate for MRI: a targeted contrast agent for FPR1 in inflammation. Chem Commun (Camb). 2013;49:564-6.

87. Chen J, Cheng H, Dong Q, Chen F, Huang Z, Zhang Y, et al. [99mTc]cFLFLF for Early Diagnosis and Therapeutic Evaluation in a Rat Model of Acute Osteomyelitis. Mol Imaging Biol. 2015;17:337-44.

88. Escalona S, Blasco JA, Reza MM, Andradas E, Gomez N. A systematic review of FDGPET in breast cancer. Med Oncol. 2010;27:114-29.

89. Groheux D, Quere G, Blanc E, Lemarignier C, Vercellino L, de Margerie-Mellon C, et al. FDG PET-CT for solitary pulmonary nodule and lung cancer: Literature review. Diagn Interv Imaging. 2016;97:1003-17.

90. Gupta T, Master Z, Kannan S, Agarwal JP, Ghsoh-Laskar S, Rangarajan V, et al. Diagnostic performance of post-treatment FDG PET or FDG PET/CT imaging in head and neck cancer: a systematic review and meta-analysis. Eur J Nucl Med Mol Imaging. 2011;38:2083-95.

91. Becker W, Bair J, Behr T, Repp R, Streckenbach H, Beck H, et al. Detection of softtissue infections and osteomyelitis using a technetium-99m-labeled anti-granulocyte monoclonal antibody fragment. J Nucl Med. 1994;35:1436-43.

92. Love C, Tronco GG, Palestro CJ. Imaging of infection and inflammation with $99 \mathrm{mTc}$ Fanolesomab. QJ Nucl Med Mol Imaging. 2006;50:113-20.

93. Shanthly N, Aruva MR, Zhang K, Mathew B, Thakur ML. 99mTc-Fanolesomab: affinity, pharmacokinetics and preliminary evaluation. Q J Nucl Med Mol Imaging. 2006;50:10412.

94. Weiner RE, Thakur ML. Imaging infection/inflammations. Pathophysiologic basis and radiopharmaceuticals. QJ Nucl Med. 1999;43:2-8.

95. Fiser SM, Tribble CG, Long SM, Kaza AK, Kern JA, Jones DR, et al. Ischemiareperfusion injury after lung transplantation increases risk of late bronchiolitis obliterans syndrome. Ann Thorac Surg. 2002;73:1041-7; discussion 47-8.

96. Eltzschig HK, Sitkovsky MV, Robson SC. Purinergic signaling during inflammation. $N$ Engl J Med. 2012;367:2322-33. 
97. Anvari F, Sharma AK, Fernandez LG, Hranjec T, Ravid K, Kron IL, et al. Tissue-derived proinflammatory effect of adenosine A2B receptor in lung ischemia-reperfusion injury. $J$ Thorac Cardiovasc Surg. 2010;140:871-7.

98. Pejman L, Omrani H, Mirzamohammadi Z, Shahbazfar AA, Khalili M, Keyhanmanesh R. The Effect of Adenosine A2A and A2B Antagonists on Tracheal Responsiveness, Serum Levels of Cytokines and Lung Inflammation in Guinea Pig Model of Asthma. $A d v$ Pharm Bull. 2014;4:131-8.

99. Zhou Y, Schneider DJ, Morschl E, Song L, Pedroza M, Karmouty-Quintana H, et al. Distinct roles for the A2B adenosine receptor in acute and chronic stages of bleomycininduced lung injury. J Immunol. 2011;186:1097-106.

100. Huerter ME, Sharma AK, Zhao Y, Charles EJ, Kron IL, Laubach VE. Attenuation of Pulmonary Ischemia-Reperfusion Injury by Adenosine A2B Receptor Antagonism. Ann Thorac Surg. 2016;102:385-93.

101. Cagnina RE, Ramos SI, Marshall MA, Wang G, Frazier CR, Linden J. Adenosine A2B receptors are highly expressed on murine type II alveolar epithelial cells. Am J Physiol Lung Cell Mol Physiol. 2009;297:L467-74.

102. Mason DP, Brown CR, Murthy SC, Vakil N, Lyon C, Budev MM, et al. Growing singlecenter experience with lung transplantation using donation after cardiac death. Ann Thorac Surg. 2012;94:406-11; discussion 11-2.

103. Valenza F, Citerio G, Palleschi A, Vargiolu A, Fakhr BS, Confalonieri A, et al. Successful Transplantation of Lungs From an Uncontrolled Donor After Circulatory Death Preserved In Situ by Alveolar Recruitment Maneuvers and Assessed by Ex Vivo Lung Perfusion. Am J Transplant. 2016;16:1312-18.

104. Boffini M, Ricci D, Bonato R, Fanelli V, Attisani M, Ribezzo M, et al. Incidence and severity of primary graft dysfunction after lung transplantation using rejected grafts reconditioned with ex vivo lung perfusion. Eur J Cardiothorac Surg. 2014;46:789-93.

105. Hoegl S, Brodsky KS, Blackburn MR, Karmouty-Quintana H, Zwissler B, Eltzschig HK. Alveolar Epithelial A2B Adenosine Receptors in Pulmonary Protection during Acute Lung Injury. J Immunol. 2015;195:1815-24.

106. Teng MW, Bowman EP, McElwee JJ, Smyth MJ, Casanova JL, Cooper AM, et al. IL-12 and IL-23 cytokines: from discovery to targeted therapies for immune-mediated inflammatory diseases. Nat Med. 2015;21:719-29.

107. Cero FT, Hillestad V, Loberg EM, Christensen G, Larsen KO, Skjonsberg OH. IL-18 and IL-12 synergy induces matrix degrading enzymes in the lung. Exp Lung Res. 2012;38:406-19.

108. Hasko G, Kuhel DG, Chen JF, Schwarzschild MA, Deitch EA, Mabley JG, et al. Adenosine inhibits IL-12 and TNF-[alpha] production via adenosine A2a receptordependent and independent mechanisms. FASEB J. 2000;14:2065-74.

109. Sharma AK, Laubach VE, Ramos SI, Zhao Y, Stukenborg G, Linden J, et al. Adenosine A2A receptor activation on $\mathrm{CD} 4+\mathrm{T}$ lymphocytes and neutrophils attenuates lung ischemia-reperfusion injury. J Thorac Cardiovasc Surg. 2010;139:474-82.

110. Inoue Y, Chen Y, Pauzenberger R, Hirsh MI, Junger WG. Hypertonic saline up-regulates A3 adenosine receptor expression of activated neutrophils and increases acute lung injury after sepsis. Crit Care Med. 2008;36:2569-75. 
111. Machuca TN, Cypel M, Zhao Y, Grasemann H, Tavasoli F, Yeung JC, et al. The role of the endothelin-1 pathway as a biomarker for donor lung assessment in clinical ex vivo lung perfusion. J Heart Lung Transplant. 2015;34:849-57.

112. Vasanthan V, Nagendran J. Compliance trumps oxygenation: Predicting quality with ex vivo lung perfusion. J Thorac Cardiovasc Surg. 2015;150:1378-9.

113. Harada M, Oto T, Otani S, Miyoshi K, Okada M, Iga N, et al. A neutrophil elastase inhibitor improves lung function during ex vivo lung perfusion. Gen Thorac Cardiovasc Surg. 2015;63:645-51.

114. Kondo T, Chen F, Ohsumi A, Hijiya K, Motoyama H, Sowa T, et al. beta2Adrenoreceptor Agonist Inhalation During Ex Vivo Lung Perfusion Attenuates Lung Injury. Ann Thorac Surg. 2015;100:480-6.

115. Biancosino C, Albert M, Linder A. Acute toxicity of irinotecan in the ex-vivo isolated perfused human lung model--high-dose therapy during isolated perfusion without acute toxic lung edema. Interact Cardiovasc Thorac Surg. 2007;6:583-7.

116. Balakrishna S, Song W, Achanta S, Doran SF, Liu B, Kaelberer MM, et al. TRPV4 inhibition counteracts edema and inflammation and improves pulmonary function and oxygen saturation in chemically induced acute lung injury. Am J Physiol Lung Cell Mol Physiol. 2014;307:L158-72.

117. Hamanaka K, Jian MY, Townsley MI, King JA, Liedtke W, Weber DS, et al. TRPV4 channels augment macrophage activation and ventilator-induced lung injury. Am J Physiol Lung Cell Mol Physiol. 2010;299:L353-62.

118. Hamanaka K, Jian MY, Weber DS, Alvarez DF, Townsley MI, Al-Mehdi AB, et al. TRPV4 initiates the acute calcium-dependent permeability increase during ventilatorinduced lung injury in isolated mouse lungs. Am J Physiol Lung Cell Mol Physiol. 2007;293:L923-32.

119. Henry CO, Dalloneau E, Perez-Berezo MT, Plata C, Wu Y, Guillon A, et al. In vitro and in vivo evidence for an inflammatory role of the calcium channel TRPV4 in lung epithelium: Potential involvement in cystic fibrosis. Am J Physiol Lung Cell Mol Physiol. 2016;311:L664-75.

120. Kunert-Keil C, Bisping F, Kruger J, Brinkmeier H. Tissue-specific expression of TRP channel genes in the mouse and its variation in three different mouse strains. $B M C$ Genomics. 2006; 7:159.

121. Lohman AW, Billaud M, Straub AC, Johnstone SR, Best AK, Lee M, et al. Expression of pannexin isoforms in the systemic murine arterial network. $J$ Vasc Res. 2012;49:405-16.

122. Sandilos JK, Chiu YH, Chekeni FB, Armstrong AJ, Walk SF, Ravichandran KS, et al. Pannexin 1, an ATP release channel, is activated by caspase cleavage of its poreassociated C-terminal autoinhibitory region. J Biol Chem. 2012;287:11303-11.

123. Adamson SE, Leitinger $N$. The role of pannexin1 in the induction and resolution of inflammation. FEBS Lett. 2014;588:1416-22.

124. Eltzschig HK, Eckle T. Ischemia and reperfusion--from mechanism to translation. Nat Med. 2011;17:1391-401.

125. Junger WG. Immune cell regulation by autocrine purinergic signalling. Nat Rev Immunol. 2011;11:201-12.

126. Isakson BE, Thompson RJ. Pannexin-1 as a potentiator of ligand-gated receptor signaling. Channels (Austin). 2014;8:118-23. 
127. Lohman AW, Leskov IL, Butcher JT, Johnstone SR, Stokes TA, Begandt D, et al. Pannexin 1 channels regulate leukocyte emigration through the venous endothelium during acute inflammation. Nat Commun. 2015;6:7965.

128. Lohman AW, Isakson BE. Differentiating connexin hemichannels and pannexin channels in cellular ATP release. FEBS Lett. 2014;588:1379-88.

129. Whitson BA, Black SM. Organ assessment and repair centers: The future of transplantation is near. World J Transplant. 2014;4:40-2. 